\title{
WestVirginiaUniversity
}

THE RESEARCH REPOSITORY @ WVU

Graduate Theses, Dissertations, and Problem Reports

2016

\section{On Supereulerian Digraphs}

Mansour Jarbo Algefari

Follow this and additional works at: https://researchrepository.wvu.edu/etd

\section{Recommended Citation}

Algefari, Mansour Jarbo, "On Supereulerian Digraphs" (2016). Graduate Theses, Dissertations, and Problem Reports. 5061.

https://researchrepository.wvu.edu/etd/5061

This Dissertation is protected by copyright and/or related rights. It has been brought to you by the The Research Repository @ WVU with permission from the rights-holder(s). You are free to use this Dissertation in any way that is permitted by the copyright and related rights legislation that applies to your use. For other uses you must obtain permission from the rights-holder(s) directly, unless additional rights are indicated by a Creative Commons license in the record and/ or on the work itself. This Dissertation has been accepted for inclusion in WVU Graduate Theses, Dissertations, and Problem Reports collection by an authorized administrator of The Research Repository @ WVU.

For more information, please contact researchrepository@mail.wvu.edu. 


\title{
On Supereulerian Digraphs
}

\author{
Mansour Jarbo Algefari \\ Dissertation submitted to the \\ Eberly College of Arts and Sciences \\ at West Virginia University \\ in partial fulfillment of the requirements \\ for the degree of \\ Doctor of Philosophy \\ in \\ Mathematics \\ Hong-Jian Lai, Ph.D., Chair \\ John Goldwasser, Ph.D. \\ Rong Luo, Ph.D. \\ K. Subramani, Ph.D. \\ Jerzy Wojciechowski, Ph.D. \\ Department of Mathematics \\ West Virginia University \\ Morgantown, West Virginia 26506 \\ 2016
}

Keywords: Strong arc connectivity, Maximum matching, Eulerian digraphs, Locally dense, Forbidden induced subdigraphs

Copyright 2016 Mansour Jarbo Algefari 


\section{ABSTRACT}

\section{On Supereulerian Digraphs}

\section{Mansour Jarbo Algefari}

A digraph $D$ is eulerian if $D$ is connected and for any $v \in V(D), d_{D}^{+}(v)=d_{D}^{-}(v)$. A digraph $D$ is supereulerian if $D$ contains a spanning eulerian subdigraph. A digraph $D$ is a closed ditrail if it is eulerian. This dissertation focuses on the sufficient conditions for a digraph to be supereulerian and we discussed it for the following conditions.

1. Matching number condition.

Let $D$ be a digraph and $G$ be a graph. Let $\lambda(D)$ be the arc-strong connectivity of $D, \alpha(D)$ be the independence number of $D, \alpha^{\prime}(D)$ be the size of a maximum matching of $D$, and $\kappa^{\prime}(G)$ be the edge conectivity of $G$. Motivated by the following conjecture: Let $D$ be a digraph. If $\lambda(D) \geq \alpha(D)$, then $D$ is supereulerian, which has been verified in several families of digraphs. There have been investigations on supereulerian properties of a graph $G$ with given inequality constraints on $\kappa^{\prime}(G), \alpha(G)$ and $\alpha^{\prime}(G)$, as seen in [1], [15], [18], [22] and [23], among others. In [5], Bang-Jensen and Maddaloni also proved that if $\kappa^{\prime}(G) \geq \alpha(G)$ for a graph $G$, then $G$ is supereulerian. We prove that if $\lambda(D) \geq \alpha^{\prime}(D)>0$, then $D$ has a spanning eulerian subdigraph.

\section{Locally density condition.}

It is well known (for example, Corollary 1 of [10]) that every connected, locally-connected graph other than $K_{2}$ is supereulerian. The problem investigated in this chapter is whether strong and locally strong digraphs will behave similarly, and how local structure in digraph will warrant the existence of a spanning eulerian subdigraph. We shall prove that for any integer $k>0$, there exists an infinite family of strong, $k^{+}$-locally-arc-connected non-supereulerian digraphs which is also an infinite family of strong, $k$-locally-arc-connected non-supereulerian digraphs. These suggest that local connectivity may not be sufficient for supereulerian digraphs. This motivates us to seek whether locally dense conditions for supereulerian digraphs. Let $\alpha, \beta$ be two rational numbers. A strong digraph $D$ is locally $(\alpha, \beta)^{+}$-arc-connected if $\forall v \in V(D)$, $\lambda\left(D\left[N^{+}(v)\right]\right) \geq \alpha\left|N^{+}(v)\right|+\beta$. A locally $(0, k)^{+}$-arc-connected digraph is also called $k^{+}$-locallyarc-connected. We prove that every locally $\left(\frac{2}{3}, 0\right)^{+}$-arc-connected strong digraph is supereulerian. 
3. Forbidden induced short dipaths condition.

We investigate forbidden induced subdigraph conditions to assure the existence of supereulerian digraphs. For an integer $k \geq 2$, let $P_{k}$ denote the dipath on $k$ vertices. A subdigraph $H$ of a digraph $D$ is a $P_{k}$-subdigraph if $H \cong P_{k}$. If $D$ does not have an induced $P_{k}$, then for any $P_{k}$-subdigraph $H$ of $D$, we must have $|A(D[V(H)])| \geq k$. For integers $h \geq k>2$, we define $\mathcal{F}\left(P_{k}, h\right)$ to be the family of all simple digraphs such that $D \in \mathcal{F}\left(P_{k}, h\right)$ if and only if $D$ is strong and satisfies both of the following.

(i) $D$ contains at least one dipath $P_{k}$ with $\left|A\left(D\left[V\left(P_{k}\right)\right]\right)\right|=h$, and

(ii) for any dipath $P_{k}$ in $D,\left|A\left(D\left[V\left(P_{k}\right)\right]\right)\right| \geq h$.

We determine the smallest integer $h_{k}$ such that if a strong digraph $D$ containing a subdigraph $H$ isomorphic to $P_{k}$ always satisfies $|A(D[V(H)])| \geq h_{k}$ with $k \in\{2,3,4\}$, then $D$ is supereulerian.

\section{Degree condition.}

The property of being supereulerian is at the same time a relaxation of being hamiltonian: being supereulerian means having a closed ditrail covering all the vertices; being hamiltonian means having a closed ditrail covering all the vertices without using a vertex twice (hamiltonian cycle). Motivated by this analogy with hamiltonian cycles, we obtain a sufficient degree condition for supereulerianity similar to that for hamiltonicity in [24] due to Zhao and Meng. We prove that let $D$ be a strong digraph. If

$$
d^{+}(x)+d^{+}(y)+d^{-}(u)+d^{-}(v) \geq 2 n-3
$$

for every pair $x, y$ of dominating non-adjacent vertices and every pair $u, v$ of dominated nonadjacent vertices, then $D$ is supereulerian.

5. Matching number and minimum degree condition for a graph.

Let $G$ be a graph. Let $\delta(G)$ be the minimum degree of $G, \kappa^{\prime}(G)$ be the edge-connectivity of $G$, $\alpha(G)$ be the independence number of $G$ and $\alpha^{\prime}(G)$ be the size of a maximum matching of $G$. Motivated by the investigations on supereulerian properties of a graph $G$ with given inequality constraints on $\kappa^{\prime}(G), \alpha(G)$ and $\alpha^{\prime}(G)$, as seen in [1], [15], [18], [22] and [23], among others. In [5], Bang-Jensen and Maddaloni proved that if $\kappa^{\prime}(G) \geq \alpha(G)$ for a graph $G$, then $G$ is supereulerian. We prove that if $\delta(G) \geq \alpha^{\prime}(G) \geq 2$, where $|V(G)|$ is even when $\alpha^{\prime}(G)=2$, then $G$ has a spanning eulerian subdigraph. 


\section{Acknowledgements}

First and foremost, I am most indebted to my supervisor, Prof. Hong-Jian Lai, for his continued encouragement and support over these last few years. It is a pleasure to work under his supervision. Without Allah then him, this paper could not have come about.

I would also like to thank my other committee members: Prof. John Goldwasser, Prof. K. Subramani, Prof. Jerzy Wojciechowski, and Prof. Rong Luo, for their help during my studies.

And finally, I would like to thank the Department of Mathematics and Eberly College of Arts

and Sciences at West Virginia University for providing me with an excellent study environment and support during my study as a graduate student. 


\section{DEDICATION}

To

my father $\underline{\text { Jarbo }, \text { my mother Latifah, my wife Sumayah }}$

and

my lovely children Sultan, Ghala, Fanan and Dana 


\section{Contents}

1 Preliminaries 1

1.1 Notation and Terminology . . . . . . . . . . . . . . . . . . 1

1.2 Previous Work . . . . . . . . . . . . . . . . . . . 3

1.3 Main Results . . . . . . . . . . . . . . . . . . . . . . 6

2 Supereulerian Digraphs with Large Arc-strong Connectivity $\quad 8$

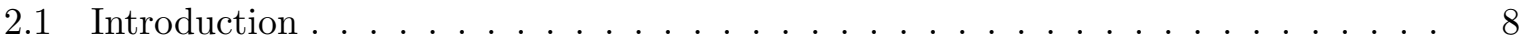

2.2 Tools . . . . . . . . . . . . . . . . . . . . . . . . 10

2.3 Sharpness of the Main Result . . . . . . . . . . . . . . . . . . . . . . . 14

2.4 Proof of the Main Result _ . . . . . . . . . . . . . . . . . . . . . 15

3 Locally Dense Supereulerian Digraphs $\quad 18$

3.1 Introduction . . . . . . . . . . . . . . . . . . . . 18

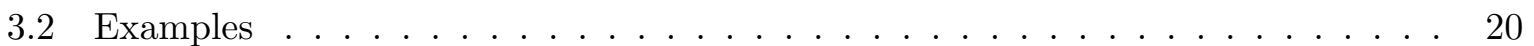

3.3 Proof of Theorem $3.1 .1 \ldots \ldots \ldots \ldots \ldots \ldots$

4 Supereulerian Digraphs with Forbidden Induced Short Dipaths 29

4.1 Introduction . . . . . . . . . . . . . . . . . . . . . . . 29 
4.2 Supereulerian Digraph in $\mathcal{F}\left(P_{2}, h\right)$ and $\mathcal{F}\left(P_{3}, h\right) \ldots \ldots \ldots$

4.3 Supereulerian Digraph in $\mathcal{F}\left(P_{4}, h\right) \ldots \ldots \ldots \ldots \ldots \ldots$

5 Degree Condition for Supereulerian Digraph 39

5.1 Introduction . . . . . . . . . . . . . . . . . . . . 39

5.2 Main Theorem . . . . . . . . . . . . . . . . . . . . 43

5.3 Sharpness of the Main Theorem . . . . . . . . . . . . . . . 45

5.4 Future Tool . . . . . . . . . . . . . . . . . . . . 46

6 Matching and Minimum Degree in Graph $\quad 49$

6.1 Introduction . . . . . . . . . . . . . . . . . . 49

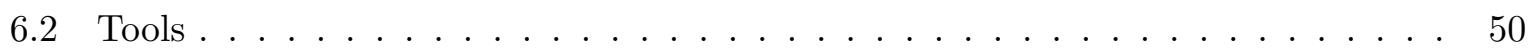

6.3 The Main Result . . . . . . . . . . . . . . . . . . . . . 55

6.4 Sharpness of the Main Result . . . . . . . . . . . . . . . . . . . 57 


\section{Chapter 1}

\section{Preliminaries}

\subsection{Notation and Terminology}

We consider finite and simple graphs and digraphs. A digraph $D$ is simple if $D$ has no loops and if for any pair of distinct vertices $u, v \in V(D)$, there is at most one arc in $D$ oriented from $u$ to $v$. Usually, we use $G$ to denote a graph and $D$ a digraph. Undefined terms and notations will follow [8] for graphs and [4] for digraphs. In particular, $\kappa(G), \kappa^{\prime}(G), \alpha(G)$ and $\alpha^{\prime}(G)$ denote the connectivity, the edge connectivity, the independence number, and the matching number of a graph $G$; and $\kappa(D)$ and $\lambda(D)$ denotes the vertex-strong connectivity and the arc-strong connectivity of a digraph $D$, respectively, $c(D)$ denotes the number of components of $G(D)$. Throughout this chapter, we use the notation $(u, v)$ to denote an arc oriented from $u$ to $v$ in a digraph; and use $[u, v]$ to denote either $(u, v)$ or $(v, u)$. When $[u, v] \in A(D)$, we say that $u$ and $v$ are adjacent. If two $\operatorname{arcs}$ of $D$ have a common vertex, we say that these two arcs are adjacent in $D$. For an integer $n>0$, we use $K_{n}^{*}$ to denote the complete digraph on $n$ vertices. Hence for every pair of distinct vertices $u, v \in V\left(K_{n}^{*}\right)$, there is exactly one arc $(u, v)$ in $A\left(K_{n}^{*}\right)$.

If $D$ is a digraph, we often use $G(D)$ to denote the underlying undirected graph of $D$, the graph obtained from $D$ by erasing all orientation on the $\operatorname{arcs}$ of $D$. A digraph $D$ is weakly connected if $G(D)$ is connected. The independence number and the matching number of a digraph $D$ are defined as

$$
\alpha(D)=\alpha(G(D)) \text { and } \alpha^{\prime}(D)=\alpha^{\prime}(G(D))
$$

respectively.

For graphs $H$ and $G$, by $H \subseteq G$ we mean that $H$ is a subgraph of $G$. Similarly, for digraphs 
$H$ and $D$, by $H \subseteq D$ we mean that $H$ is a subdigraph of $D$. If $X \subseteq V(D) \cup A(D)$, then $D[X]$ denotes the subdigraph induced by $X$. For a subdigraph $S$ of a digraph $D$, and for subsets $X \subseteq A(S)$ and $Y \subseteq A(D)-A(S)$, we use $S-X+Y$ to denote $D[(A(S)-X) \cup Y]$, the subdigraph induced by the arc subset $(A(S)-X) \cup Y$. Following [4], for a digraph $D$ with $X, Y \subseteq V(D)$, define

$$
(X, Y)_{D}=\{(x, y) \in A(D): x \in X, y \in Y\} .
$$

When $Y=V(D)-X$, we define

$$
\partial_{D}^{+}(X)=(X, V(D)-X)_{D} \text { and } \partial_{D}^{-}(X)=(V(D)-X, X)_{D}
$$

For a vertex $v \in V(D), d_{D}^{+}(v)=\left|\partial_{D}^{+}(\{v\})\right|$ and $d_{D}^{-}(v)=\left|\partial_{D}^{-}(\{v\})\right|$ are the out-degree and the indegree of $v$ in $D$, respectively. Finally, we define the following notations: $\delta^{+}(D)=\min \left\{d_{D}^{+}(v)\right.$ : $v \in V(D)\}, \delta^{-}(D)=\min \left\{d_{D}^{-}(v): v \in V(D)\right\}$. For any vertex $v \in V(D)$, define

$$
\partial_{D}(v)=\partial_{D}^{+}(v) \cup \partial_{D}^{-}(v) \text {, and } d_{D}(v)=d_{D}^{+}(v)+d_{D}^{-}(v) .
$$

For any vertex $v \in V(D)$, we define the out neighbors of $v$ by $N^{+}(v)=\{u: u \in V(D)$ and $(v, u) \in A(D)\}$, the in neighbors of $v$ by $N^{-}(v)=\{u: u \in V(D)$ and $(u, v) \in A(D)\}$ and the neighbors of $v$ by $N(v)=N^{+}(v) \cup N^{-}(v)$. When the digraph $D$ is understood from the context, we often omit the subscript $D$. By the definition of $\lambda(D)$ in [4], we note that for any integer $k \geq 0$ and a digraph $D$,

$$
\lambda(D) \geq k \text { if and only if for any nonempty proper subset } X \subset V(D),\left|\partial_{D}^{+}(X)\right| \geq k \text {. }
$$

Following the definition in [4] (Page 11), for a subdigraph $H$ of a digraph $D$, an $(x, y)$-dipath $P$ is an $(H, H)$-dipath if $x, y \in V(H)$ and $V(P) \cap V(H)=\{x, y\}$. A digraph $D$ is semicomplete if there is an arc between every pair of vertices in $D$. A digraph $D$ is locally in-semicomplete (locally out-semicomplete) if, for every vertex $x$ of $D$, the in-neighbours (out-neighbours) of $x$ induce a semicomplete digraph. A digraph which is both locally in-semicomplete and locally out-semicomplete is locally semicomplete.

A digraph $D=(V, A)$ is semicomplete multipartite if there is a partition $V_{1}, V_{2}, \ldots, V_{c}$ of $V$ into independent sets so that every vertex in $V_{i}$ shares an arc with every vertex in $V_{j}$ for $1 \leq i<j \leq c$. A digraph $D$ is quasi - transitive if, for any triple $x, y, z$ of distinct vertices of $D$ such that $(x, y)$ and $(y, z)$ are $\operatorname{arcs}$ of $D$, there is at least one arc between $x$ and $z$.

Motivated by the Chinese Postman Problem, Boesch, Suffel, and Tindell [7] in 1977 proposed the supereulerian problem, which seeks to characterize graphs that have spanning Eulerian subgraphs, and they [7] indicated that this problem would be very difficult. Pulleyblank [20] later in 1979 proved that determining whether a graph is supereulerian, even within planar 
graphs, is NP-complete. Since then, there have been lots of researches on this topic. Catlin [9] in 1992 presented the first survey on supereulerian graphs. Later Chen et al [11] gave an update in 1995, specifically on the reduction method associated with the supereulerian problem. A recent survey on supereulerian graphs is given in [17].

It is a natural to consider the supereulerian problem in digraphs. A digraph $D$ is eulerian if $D$ is connected and for any $v \in V(D), d_{D}^{+}(v)=d_{D}^{-}(v)$. A digraph $D$ is supereulerian if $D$ contains a spanning eulerian subdigraph. A digraph $D$ is a closed ditrail if it is eulerian. The main problem is to determine supereulerian digraphs.

\subsection{Previous Work}

Several efforts in supereulerian digraphs have been made. However, contrary to the case of undirected graphs, not much work has been done yet for supereulerian digraphs. The earlier studies were done by Gutin ( $[13,14])$. In [14], G.Gutin stated the following definitions:

Definition 1.2.1. Given a digraph $D$ and two positive integers $f(x), g(x)$ for every $x \in V(D), a$ subgraph $H$ of $D$ is called a $(g, f)$-factor if $g(x) \leq d_{H}^{+}(x)=d_{H}^{-}(x) \leq f(x)$ for every $x \in V(D)$. If $f(x)=g(x)=1$ for every $x$, then a connected $(g, f)$-factor is a hamiltonian cycle.

Definition 1.2.2. An extension of a digraph $D$ is a new digraph $H$ obtained from $D$ by replacing every vertex $x \in V(D)$ with a set of independent vertices $S_{x}$ such that, for every pair of distinct $x, y \in V(D)$, an arc $(u, v)$, where $u \in S_{x}, v \in S_{y}$, is in $H$ if and only if $(x, y)$ is in $D$. An extension of a locally in-semicomplete digraph is called an extended locally in - semicomplete digraph.

Then he proved the following theorem:

Theorem 1.2.3. (Gutin [14]) Let D be a semicomplete bipartite digraph or an extended locally in - semicomplete digraph. Then $D$ has a connected $(g, f)$-factor if and only if $D$ is strongly connected and contains a $(g, f)$-factor.

Using definition1.2.1 Gutin added that: Clearly, a digraph $H$ is supereulerian if and only if $H$ has a connected $\left(1, d^{*}\right)$-factor, where $d^{*}=\min \left\{d^{+}(x), d^{-}(x)\right\}$ for every $x \in V(H)$. As a result he presented the following corollary:

Corollary 1.2.4. (Gutin [14]) Let D be a semicomplete bipartite digraph or an extended locally in - semicomplete digraph. $D$ is supereulerian if and only if $D$ is strongly connected and contains a $\left(1, d^{*}\right)$-factor. 
A well known theorem of Chvátal and Erdös [12] states that if $|V(G)| \geq 3$ and if $\kappa(G) \geq$ $\alpha(G)$, then $G$ is hamiltonian. Thomassen [21] gave an infinite family of non hamiltonian (but supereulerian) digraphs such that $\kappa(D)=\alpha(D)=2$, showing that the Chvátal-Erdös Theorem does not extend to digraphs. This motivates Bang-Jensen and Thommassé in [5] to make the following conjecture.

Conjecture 1.2.5. Let $D$ be a digraph. If $\lambda(D) \geq \alpha(D)$, then $D$ is supereulerian.

Moreover in [5] Bang-Jensen and Alessandro Maddaloni analyzed a number of sufficient conditions for a digraph to be supereulerian starting with the following definition:

Definition 1.2.6. Given a digraph $D$, a dicycle factor, eulerian factor of $D$ is a collection of vertex-disjoint, arc-disjoint, respectively dicycles spanning $V(D)$.

Then as an effort towards conjecture 1.2.5 they proved the following theorem:

Theorem 1.2.7. (J. Bang-Jensen and A. Maddaloni [5]) Let $D$ be a digraph. If $\lambda(D) \geq \alpha(D)$ then $D$ has an eulerian factor.

In [5], Conjecture 1.2.5 has been verified in several families of digraphs. Gutin in [13] proved the following theorem:

Theorem 1.2.8. (Gutin [13]) An extended semicomplete digraph is hamiltonian if and only if it is strong and has a cycle factor.

Using a similar approach to Gutin's proof of Theorem 1.2.8, Bang-Jensen and Alessandro Maddaloni in [5] proved the following supereulerian version of it.

Theorem 1.2.9. (J. Bang-Jensen and A. Maddaloni [5]) Let D be a semicomplete multipartite digraph. $D$ is supereulerian if and only if it is strong and has an eulerian factor.

Combining Theorem 1.2.9 and Theorem 2.2.2, Bang-Jensen and Alessandro Maddaloni verify Conjecture 1.2.5 for semicomplete multipartite digraphs.

Theorem 1.2.10. (J. Bang-Jensen and A. Maddaloni [5]) Let D be a semicomplete multipartite digraph. If $\lambda(D) \geq \alpha(D)$, then $D$ is supereulerian.

In addition, Bang-Jensen and Alessandro Maddaloni proved that:

Theorem 1.2.11. (J. Bang-Jensen and A. Maddaloni [5]) Let D be a quasi transitive digraph. If $\lambda(D) \geq \alpha(D)$, then $D$ is supereulerian. 
Also, Bang-Jensen and Alessandro Maddaloni proved the following:

Theorem 1.2.12. (J. Bang-Jensen and A. Maddaloni [5]) A strong digraph such that $d(x)+$ $d(y) \geq 2 n-3$ for all pairs of non-adjacent vertices $x, y$ is supereulerian.

Corollary 1.2.13. (J. Bang-Jensen and A. Maddaloni [5]) A strong digraph such that $d^{+}(x)+$ $d^{-}(y) \geq n-1$ for all ordered pairs $(x, y)$ of non-adjacent vertices is supereulerian.

Finally, Bang-Jensen and Alessandro Maddaloni proved that the condition in congecture 1.2.5 is not necesary by the following example: consider a directed cycle on 4 vertices $C_{4}$ : it is eulerian, and hence supereulerian, but $\lambda\left(C_{4}\right)=1$ and $\alpha\left(C_{4}\right)=2$. Moreover, they provided infinite families of digraphs with $\lambda(D)=\alpha(D)-1$ that are not supereulerian. Hence, if true, Congecture 1.2.5 would be best possible.

In [16], Yanmei Hong, Hong-Jian Lai and Qinghai Liu after producing the following definition:

Definition 1.2.14. Let $D$ be a strong digraph and $U \subsetneq V(D)$. Then in $D[U]$, we can find some ditrails $P_{1}, \ldots, P_{t}$ such that $\cup_{i=1}^{t} V\left(P_{i}\right)=U$ and $A\left(P_{i}\right) \cap A\left(P_{j}\right)=\emptyset$ for any $i \neq j$. Let $\tau(U)$ be minimum value of such $t$. Then $c(G(D[U])) \leq \tau(U) \leq|U|$, where $c(G(D[U]))$ is the number of component of the underlying graph of $D[U]$. For any $A \subseteq V(D)-U$, denote $B:=V(D)-U-A$ and let

$$
\begin{aligned}
h(U, A): & =\min \left\{\left|\partial_{D}^{+}(A)\right|,\left|\partial_{D}^{-}(A)\right|\right\}+\min \left\{\left|(U, B)_{D}\right|,\left|(B, U)_{D}\right|\right\}-\tau(U), \text { and } \\
h(U): & =\min \{h(U, A): A \cap U=\emptyset\} .
\end{aligned}
$$

Yanmei Hong, Hong-Jian Lai and Qinghai Liu gave the following necessary condition for the existence of a spanning eulerian subdigraph:

Proposition 1.2.15. (Proposition (2.1) of Hong et al [16]) If D has a spanning eulerian subdigraph, then for any $U \subset V(D), h(U) \geq 0$.

Moreover, Hong et al gave the following sufficient degree condition for the existence of a spanning eulerian subdigraph:

Theorem 1.2.16. (Hong et al [16]) Let $D$ be a strong digraph of order $n$ and minimum outdegree $\delta^{+} \geq 4$ and minimum in-degree $\delta^{-} \geq 4$. If $\delta^{+}+\delta^{-}>n-4$, then $D$ has a spanning eulerian subdigraph. 


\subsection{Main Results}

The main results in the dissertation are summarized as follows.

1. Let $D$ be a digraph and $G$ be a graph. Let $\lambda(D)$ be the arc-strong connectivity of $D, \alpha(D)$ be the independence number of $D, \alpha^{\prime}(D)$ be the size of a maximum matching of $D$, and $\kappa^{\prime}(G)$ be the edge conectivity of $G$. In Chapter 2 motivated by Conjecture 1.2.5 we prove that if $\lambda(D) \geq \alpha^{\prime}(D)>0$, then $D$ has a spanning eulerian subdigraph.

2. It is well known (for example, Corollary 1 of [10]) that every connected, locally-connected graph other than $K_{2}$ is supereulerian. The problem investigated in Chapter 3 is whether strong and locally strong digraphs will have similar properties as graphs; and how local structure in digraph will warrant the existence of a spanning eulerian subdigraph. We shall prove that for any integer $k>0$, there exists an infinite family of strong, $k^{+}$ locally-arc-connected non-supereulerian digraphs which is also an infinite family of strong, $k$-locally-arc-connected non-supereulerian digraphs. These suggest that local connectivity may not be sufficient for supereulerian digraphs. This motivates us to seek whether locally dense conditions for supereulerian digraphs. Let $\alpha, \beta$ be two rational numbers. A strong digraph $D$ is locally $(\alpha, \beta)^{+}$-arc-connected if $\forall v \in V(D), \lambda\left(D\left[N^{+}(v)\right]\right) \geq \alpha\left|N^{+}(v)\right|+\beta$. A locally $(0, k)^{+}$-arc-connected digraph is also called $k^{+}$-locally-arc-connected. We prove that every locally $\left(\frac{2}{3}, 0\right)^{+}$-arc-connected strong digraph is supereulerian.

3. We investigate forbidden induced subdigraph conditions to assure the existence of supereulerian digraphs. For an integer $k \geq 2$, let $P_{k}$ denote the dipath on $k$ vertices. A subdigraph $H$ of a digraph $D$ is a $P_{k}$-subdigraph if $H \cong P_{k}$. If $D$ does not have an induced $P_{k}$, then for any $P_{k}$-subdigraph $H$ of $D$, we must have $|A(D[V(H)])| \geq k$. For integers $h \geq k>2$, we define $\mathcal{F}\left(P_{k}, h\right)$ to be the family of all simple digraphs such that $D \in \mathcal{F}\left(P_{k}, h\right)$ if and only if $D$ is strong and satisfies both of the following.

(i) $D$ contains at least one dipath $P_{k}$ with $\left|A\left(D\left[V\left(P_{k}\right)\right]\right)\right|=h$, and

(ii) for any dipath $P_{k}$ in $D,\left|A\left(D\left[V\left(P_{k}\right)\right]\right)\right| \geq h$.

We determine the smallest integer $h_{k}$ such that if a strong digraph $D$ containing a subdigraph $H$ isomorphic to $P_{k}$ always satisfies $|A(D[V(H)])| \geq h_{k}$ with $k \in\{2,3,4\}$, then $D$ is supereulerian.

4. The property of being supereulerian can be viewed as a relaxation of being hamiltonian: 
being supereulerian means having a closed ditrail covering all the vertices, while being hamiltonian means having a closed ditrail covering all the vertices without using a vertex twice (hamiltonian cycle). Motivated by this analogy with hamiltonian cycles, we obtain a sufficient degree condition for supereulerianity similar to that for hamiltonicity in [24] due to Zhao and Meng. We prove that let $D$ be a strong digraph. If

$$
d^{+}(x)+d^{+}(y)+d^{-}(u)+d^{-}(v) \geq 2 n-3
$$

for every pair $x, y$ of dominating non-adjacent vertices and every pair $u, v$ of dominated non-adjacent vertices, then $D$ is supereulerian.

5. Let $G$ be a graph. Let $\delta(G)$ be the minimum degree of $G, \kappa^{\prime}(G)$ be the edge-connectivity of $G, \alpha(G)$ be the independence number of $G$ and $\alpha^{\prime}(G)$ be the size of a maximum matching of $G$. Motivated by the investigations on supereulerian properties of a graph $G$ with given inequality constraints on $\kappa^{\prime}(G), \alpha(G)$ and $\alpha^{\prime}(G)$, as seen in [1], [15], [18], [22] and [23], among others. In [5], Bang-Jensen and Maddaloni proved that if $\kappa^{\prime}(G) \geq \alpha(G)$ for a graph $G$, then $G$ is supereulerian. We prove that if $\delta(G) \geq \alpha^{\prime}(G) \geq 2$, where $|V(G)|$ is even when $\alpha^{\prime}(G)=2$, then $G$ has a spanning eulerian subdigraph. 


\section{Chapter 2}

\section{Supereulerian Digraphs with Large Arc-strong Connectivity}

\section{$2.1 \quad$ Introduction}

We consider finite graphs and finite and simple digraphs. Usually, we use $G$ to denote a graph and $D$ a digraph. Undefined terms and notations will follow [8] for graphs and [4] for digraphs. In particular, $\kappa(G), \kappa^{\prime}(G), \alpha(G)$ and $\alpha^{\prime}(G)$ denote the connectivity, the edge connectivity, the independence number, and the matching number of a graph $G$; and $\kappa(D)$ and $\lambda(D)$ denotes the vertex-strong connectivity and the arc-strong connectivity of a digraph $D$, respectively. Throughout this chapter, we use the notation $(u, v)$ to denote an arc oriented from $u$ to $v$ in a digraph; and use $[u, v]$ to denote either $(u, v)$ or $(v, u)$. When $[u, v] \in A(D)$, we say that $u$ and $v$ are adjacent. If two $\operatorname{arcs}$ of $D$ have a common vertex, we say that these two arcs are adjacent in $D$.

If $D$ is a digraph, we often use $G(D)$ to denote the underlying undirected graph of $D$, the graph obtained from $D$ by erasing all orientation on the arcs of $D$. The independence number and the matching number of a digraph $D$ are defined as

$$
\alpha(D)=\alpha(G(D)) \text { and } \alpha^{\prime}(D)=\alpha^{\prime}(G(D))
$$

respectively.

For graphs $H$ and $G$, by $H \subseteq G$ we mean that $H$ is a subgraph of $G$. Similarly, for digraphs $H$ and $D$, by $H \subseteq D$ we mean that $H$ is a subdigraph of $D$. Following [4], for a digraph $D$ with 
$X, Y \subseteq V(D)$, define

$$
(X, Y)_{D}=\{(x, y) \in A(D): x \in X, y \in Y\}
$$

When $Y=V(D)-X$, we define

$$
\partial_{D}^{+}(X)=(X, V(D)-X)_{D} \text { and } \partial_{D}^{-}(X)=(V(D)-X, X)_{D}
$$

For a vertex $v \in V(D), d_{D}^{+}(v)=\left|\partial_{D}^{+}(\{v\})\right|$ and $d_{D}^{-}(v)=\left|\partial_{D}^{-}(\{v\})\right|$ are the out-degree and the indegree of $v$ in $D$, respectively. Finally, we define the following notations: $\delta^{+}(D)=\min \left\{d_{D}^{+}(v)\right.$ : $v \in V(D)\}$ and $\delta^{-}(D)=\min \left\{d_{D}^{-}(v): v \in V(D)\right\}$. For any vertex $v \in V(D)$, define

$$
\partial_{D}(v)=\partial_{D}^{+}(v) \cup \partial_{D}^{-}(v) \text {, and } d_{D}(v)=d_{D}^{+}(v)+d_{D}^{-}(v)
$$

When the digraph $D$ is understood from the context, we often omit the subscript $D$. By the definition of $\lambda(D)$ in [4], we note that for any integer $k \geq 0$ and a digraph $D$,

$$
\lambda(D) \geq k \text { if and only if for any nonempty proper subset } X \subset V(D),\left|\partial_{D}^{+}(X)\right| \geq k \text {. }
$$

A well known theorem of Chvátal and Erdös [12] states that if $|V(G)| \geq 3$ and if $\kappa(G) \geq$ $\alpha(G)$, then $G$ is hamiltonian. Thomassen [21] gave an infinite family of non hamiltonian (but supereulerian) digraphs such that $\kappa(D)=\alpha(D)=2$, showing that the the Chvátal-Erdös Theorem does not extend to digraphs. This motivates Bang-Jensen and Thommassé in [5] to make the following conjecture.

Conjecture 2.1.1. Let $D$ be a digraph. If $\lambda(D) \geq \alpha(D)$, then $D$ is supereulerian.

In [5], Conjecture 2.1.1 has been verified in several families of digraphs. There have been investigations on supereulerian properties of a graph $G$ with given inequality constraints on $\kappa^{\prime}(G), \alpha(G)$ and $\alpha^{\prime}(G)$, as seen in [1], [15], [18], [22] and [23], among others. In [5], Bang-Jensen and Maddaloni also proved that if $\kappa^{\prime}(G) \geq \alpha(G)$ for a graph $G$, then $G$ is supereulerian. This motivates us to present and prove the following main result of this chapter.

Theorem 2.1.2. Let $D$ be a strong digraph. If $\lambda(D) \geq \alpha^{\prime}(D)$, then $D$ is supereulerian.

The following corollary is immediate.

Corollary 2.1.3. Let $D$ be a strong digraph. If $\kappa(D) \geq \alpha^{\prime}(D)$, then $D$ is supereulerian.

In the next section, we present some of the former theorems and develop a few lemmas which will be used in our arguments. The proof of the main result will be given in the last section. 


\subsection{Tools}

In this section, we present some tools needed in our arguments. Let $M$ be a matching in a graph $G$. We use $V(M)$ to denote the set of vertices in $G$ that are incident with an edge in $M$. (Similarly, we define $V(M)$ if $M$ is a matching in a digraph $D$ ). A path $P$ is an $M$-augmenting path if the edges of $P$ are alternately in $M$ and in $E(G)-M$, and if both end vertices of $P$ are not in $V(M)$. The following theorem is fundamental.

Theorem 2.2.1. (Berge, [6]) A matching $M$ in $G$ is a maximum matching if and only if $G$ does not have $M$-augmenting paths.

The next theorem is on hamiltonian digraphs, will also be needed in our proofs. Note that hamiltonian digraphs are also supereulerian digraphs.

Theorem 2.2.2. (Meyniel [19]) A strong digraph $D$ on $n$ vertices satisfying $d(x)+d(y) \geq 2 n-1$ for all pairs of non-adjacent vertices $x, y$ is hamiltonian.

Two more lemmas will also be needed. Throughout the rest of this section, $D$ always denotes a digraph.

Lemma 2.2.3. Let $k>0$ be an integer and $D$ be a digraph with a matching $M$ such that $|M|=k$. Suppose that $V(D)-V(M)$ has a subset $X$ with $|X| \geq 2$ such that for any $v \in X$, $d(v) \geq 2 k-1$. If $X$ has at least one vertex $u$ such that $d(u) \geq 2 k+1$, then there exists a matching $M^{\prime}$ in $D$ such that $|M|<\left|M^{\prime}\right|$.

Proof. By contradiction, we assume that $M$ is a maximum matching in $D$. By Theorem 2.2.1, $D$ has no $M$-augmenting path. Let $u, v \in X$ be distinct vertices such that $d(u) \geq 2 k+1$. Since $M$ is maximum, $u$ and $v$ are not adjacent in $D$, and so any vertices adjacent to $u$ or $v$ must be in $V(M)$. Since $D$ has no $M$-augmenting path, we have the following observations.

(A) For each arc $e=[x, y] \in M$, exactly one in $\{[u, x],[v, y]\}$ can be in $A(D)$, and exactly one in $\{[u, y],[v, x]\}$ can be in $A(D)$. If not, by symmetry, we may assume that $[u, x],[v, y] \in A(D)$, and so $\{[u, x],[x, y],[v, y]\}$ induces an $M$-augmenting path in $D$.

(B) Since $d(u) \geq 2 k+1$, we may assume that $M$ has an $\operatorname{arc} e^{\prime}=\left[x^{\prime}, y^{\prime}\right]$ such that $\left[u, x^{\prime}\right],\left[u, y^{\prime}\right] \in$ $A(D)$.

(C) From Observation (A), for each arc $e=[x, y] \in M$, if $[u, x] \in A(D)$ and $[u, y] \in A(D)$, then $[v, x],[v, y] \notin A(D)$, and so $\left|(\{u, v\},\{x, y\})_{D} \cup(\{x, y\},\{u, v\})_{D}\right| \leq 4$.

(D) From Observation (A), for each $\operatorname{arc} e=[x, y] \in M$, if $[u, x] \in A(D)$ and $[v, x] \in A(D)$, then 
$[u, y],[v, y] \notin A(D)$, and so $\left|(\{u, v\},\{x, y\})_{D} \cup(\{x, y\},\{u, v\})_{D}\right| \leq 4$.

(E) From Observation (A), for each arc $e=[x, y] \in M$, if $[v, x] \in A(D)$ and $[v, y] \in A(D)$, then $[u, x],[u, y] \notin A(D)$, and so $\left|(\{u, v\},\{x, y\})_{D} \cup(\{x, y\},\{u, v\})_{D}\right| \leq 4$.

It follows from Observations (C), (D) and (E) that $|\partial(u) \cup \partial(v)| \leq 4|M|$. As $d(v) \geq 2 k-1$ and $d(u) \geq 2 k+1$, we have

$$
4 k=(2 k-1)+(2 k+1) \leq|\partial(u) \cup \partial(v)| \leq 4|M|=4 k .
$$

This implies that every $\operatorname{arc}$ in $M$ is adjacent to exactly $4 \operatorname{arcs}$ in $\partial_{D}(u) \cup \partial_{D}(v)$. From Observation (B) and by the fact that $D$ has no $M$-augmenting path, we must have $\left[v, x^{\prime}\right],\left[v, y^{\prime}\right] \notin A(D)$, and so $v$ can only be adjacent to $V(M)-\left\{x^{\prime}, y^{\prime}\right\}$. As $d(v) \geq 2 k-1=2(k-1)+1$, there must be an $\operatorname{arc}\left[x^{\prime \prime}, y^{\prime \prime}\right] \in M$ such that $\left[v, x^{\prime \prime}\right],\left[v, y^{\prime \prime}\right] \in A(D)$. Define

$$
M_{u}=\{[x, y] \in M:[u, x],[u, y] \in A(D)\} \text { and } M_{v}=\{[x, y] \in M:[v, x],[v, y] \in A(D)\} .
$$

Since $D$ has no $M$-augmenting path, $M_{u} \cap M_{v}=\emptyset$. Let $M^{\prime}=M-\left(M_{u} \cup M_{v}\right)$. Again by the fact that $D$ has no $M$-augmenting path, for each $\operatorname{arc} e=[x, y] \in M^{\prime}$, at most one end of $e$ is adjacent to vertices in $\{u, v\}$, and so

$$
\left|(\{u\},\{x, y\})_{D} \cup(\{x, y\},\{u\})_{D}\right| \leq 2 \text { and }\left|(\{v\},\{x, y\})_{D} \cup(\{x, y\},\{v\})_{D}\right| \leq 2 .
$$

It follows that $4\left|M_{u}\right|+2\left|M^{\prime}\right| \geq d(u) \geq 2 k+1$ and $4\left|M_{v}\right|+2\left|M^{\prime}\right| \geq d(v) \geq 2 k-1$. Since $4\left|M_{u}\right|+2\left|M^{\prime}\right|$ is even, we must have $4\left|M_{u}\right|+2\left|M^{\prime}\right| \geq 2 k+2$. It follows that

$$
4 k=4|M|=\left(4\left|M_{u}\right|+2\left|M^{\prime}\right|\right)+\left(4\left|M_{v}\right|+2\left|M^{\prime}\right|\right) \geq(2 k+2)+(2 k-1)=4 k+1,
$$

a contradiction. This proves the lemma.

Corollary 2.2.4. For every digraph $D, \lambda(D) \leq 2 \alpha^{\prime}(D)$.

Proof. Let $\alpha^{\prime}(D)=k$. By contradiction, we assume that $\lambda(D) \geq 2 k+1$. Hence $|V(D)| \geq$ $2 k+2$. Let $M$ denote a maximum matching of $D$. Then $|V(D)-V(M)| \geq 2 k+2-2 k=2$. Since $\lambda(D) \geq 2 k+1$, for every vertex $u \in V(D)-V(M), d(u) \geq 2 k+1$. It follows by Lemma 2.2.3 that $M$ is not a maximum matching of $D$, and so a contradiction obtains.

Lemma 2.2.5. Let $k>0$ be an integer, $D$ be a digraph on $n \geq 2 k+2$ vertices and $M$ be a maximum matching of $D$ with $|M|=k$. Suppose that

$$
\min \left\{d^{+}(v), d^{-}(v)\right\} \geq k, \text { for every vertex } v \in V(D) .
$$

Let $X=V(D)-V(M)$. Then

$$
\text { for every } x \in X \text {, we have } d^{+}(x)=d^{-}(x)=k \text {. }
$$


Moreover, if $n \geq 2 k+3$ or if $n=2 k+2$ and $D$ is strong, then for every $e=[u, v] \in M$ and for every $x \in X$, we have the following conclusions.

(i) There exists exactly one $v(e) \in\{u, v\}$ such that both $(v(e), x)$ and $(x, v(e))$ are in $A(D)$, and the vertex $u(e) \in\{u, v\}-\{v(e)\}$ is not adjacent to any vertex in $X$.

(ii) The set $\{u(e): e \in M\}$ is an independent set in $D$ such that $d^{+}(u(e))=d^{-}(u(e))=k$ for any $e \in M$ and such that for any $e, e^{\prime} \in M,\left(u(e), v\left(e^{\prime}\right)\right),\left(v\left(e^{\prime}\right), u(e)\right) \in A(D)$.

Proof. Let $N(X)$ denote the set of vertices in $D$ that is adjacent to a vertex in $X$. Since $M$ is a maximum matching, by Theorem 2.2.1

$D$ does not have an $M$-augmenting path.

By (2.4), we have $N(X) \subseteq V(M)$.

Since $n \geq 2 k+2$ and $|M|=k$, we have $|X| \geq 2$. Since $M$ is a maximum matching, it follows by (2.2) and by Lemma 2.2.3 that (2.3) must hold. In the rest of the proof for this lemma, we consider two cases.

Case 1. $\quad D$ is a digraph with $n=|V(D)| \geq 2 k+3$.

Let $m=|X|$. Then $m \geq 3$. By (2.3),

$$
\left|(X, V(M))_{D}\right|=m k=\left|(V(M), X)_{D}\right| .
$$

We further observed that, by (2.4),

for any $e \in M$, the vertices of $e$ are incident with at most $2 m$ arcs in $(X, V(M))_{D} \cup(V(M), X)_{D}$.

Let $e=[u, v] \in M$. If $u, v \in N(X)$, then by (2.4), there must be a unique $x \in X$ such that $x$ is adjacent to both $u$ and $v$; and for any $x^{\prime} \in X-\{x\}, x^{\prime}$ is not adjacent to either $u$ nor $v$. By (2.3), $\left|(\{x\}, V(M))_{D} \cup(V(M),\{x\})_{D}\right|=2 k$. As $\left|(\{x\},\{u, v\})_{D} \cup(\{u, v\},\{x\})_{D}\right| \leq 4$, it follows by $(2.5)$ and $m \geq 3$ that

$$
\begin{aligned}
\left|(X, V(M)-\{u, v\})_{D}\right|+\left|(V(M)-\{u, v\}, X)_{D}\right| & \geq 2 k(m-1)+(2 k-4)=2 k m-4 \\
& \geq 2 k m-2 m+2=2 m(k-1)+2>2 m(k-1)+1 .
\end{aligned}
$$

If $k>1$, then there must be an arc $a \in M-\{e\}$ such that the vertices of $a$ are incident with at least $2 m+1$ edges in $(X, V(M))_{D} \cup(V(M), X)_{D}$, contrary to (2.6). Hence in this case we must have $k=1$ and $M=\{e\}$. As $|X| \geq 3$, it follows from (2.5) with $m \geq 3$ that $D$ must have an $M$-augmenting path, contrary to (2.4). Hence for any $e=[u, v] \in M$, exactly one of $u$ or $v$ is adjacent to vertices in $X$. 
Thus for each $e \in M$, let $v(e)$ denote the unique vertex of $e$ that is adjacent to vertices in $X$ and $u(e)$ the other vertex of $e$ which is not adjacent to any vertex in $X$. Then as $|X|=m$, $\left|(v(e), X)_{D} \cup(X, v(e))_{D}\right| \leq 2 m$. It follows from (2.5) that

$$
2 k m \geq \sum_{e \in M}\left|(v(e), X)_{D} \cup(X, v(e))_{D}\right|=\left|(X, V(M))_{D}\right|+\left|(V(M), X)_{D}\right|=2 m k .
$$

This implies that for each $x \in X$ and for each $e \in M$, both $(x, v(e))$ and $(v(e), x)$ are in $A(D)$. This proves (i) for Case 1.

Let $Y=\{u(e): e \in M\}$ and we shall show that $Y$ is an independent set. In fact, if for some $e_{1}, e_{2} \in M,\left[u\left(e_{1}\right), u\left(e_{2}\right)\right] \in A(D)$, then for any distinct $x_{1}, x_{2} \in X,\left\{\left[x_{1}, v\left(e_{1}\right)\right], e_{1},\left[u\left(e_{1}\right), u\left(e_{2}\right)\right]\right.$, $\left.e_{2},\left[x_{2}, v\left(e_{2}\right)\right]\right\}$ induces an $M$-augmenting path, contrary to (2.4). Thus each $u(e) \in Y$ can only be adjacent to vertices in $\{v(e): e \in M\}$. As $|\{v(e): e \in M\}|=k$, by (2.2), we conclude that for each $e \in M, d^{+}(u(e))=d^{-}(u(e))$, and for any $e, e^{\prime} \in M,\left(u(e), v\left(e^{\prime}\right)\right),\left(v\left(e^{\prime}\right), u(e)\right) \in A(D)$. This proves (ii) for Case 1 .

Case 2. $\quad D$ is strong and $|V(D)|=2 k+2$.

Then $X=\{w, z\}$. Let $M=\left\{e_{1}, \cdots, e_{k}\right\}$. Let $M_{w} \subseteq M$ denote the $\operatorname{arcs}$ in $M$ each of which has a vertex adjacent to $w$. We define $M_{z}$ similarly. By (2.3), $\left|M_{w}\right| \geq \frac{k}{2}$ and $\left|M_{z}\right| \geq \frac{k}{2}$.

Subcase 1. $\left|M_{w}\right|=\frac{k}{2}$ or $\left|M_{z}\right|=\frac{k}{2}$.

Note that in this case, $k$ must be even. We assume, without loss of generality, that $M_{w}=$ $\left\{e_{1}, \cdots, e_{\frac{k}{2}}\right\}$. By (2.4), we must have $M_{z}=\left\{e_{\frac{k}{2}+1}, \cdots, e_{k}\right\}$. Again by $(2.4)$, for each $x \in V\left(M_{w}\right)$ and $y \in V\left(M_{z}\right)$, we conclude that $[x, y] \notin A(D)$. Thus

$$
\left(V\left(M_{w}\right) \cup\{w\}, V\left(M_{z}\right) \cup\{z\}\right)_{D} \cup\left(V\left(M_{z}\right) \cup\{z\}, V\left(M_{w}\right) \cup\{w\}\right)_{D}=\emptyset,
$$

contrary to the assumption that $D$ is strong. This shows that Subcase 1 cannot occur.

Subcase 2. $\left|M_{w}\right|>\frac{k}{2}$ and $\left|M_{z}\right|>\frac{k}{2}$.

Therefore, $M_{w} \cap M_{z} \neq \emptyset$. Note that by (2.4), if an arc $e \in M$ whose vertices are adjacent to both $w$ and $z$, then exactly one vertex of $e$ can be adjacent to both $w$ and $z$. Let $M^{\prime}=$ $M_{w} \cap M_{z}=\left\{e_{i}^{\prime}=\left[x_{i}, y_{i}\right],(i=1, \ldots, d ; 1 \leq d \leq k)\right\} \subseteq M$. Without loss of generality, we assume that each $e_{i}^{\prime}$ has a unique vertex $x_{i}$ with $\left[x_{i}, w\right],\left[x_{i}, z\right] \in A(D)$. Let $M^{\prime \prime}=M-M^{\prime}=\left\{e_{j}^{\prime \prime}=\right.$ $\left.\left[r_{j}, s_{j}\right],(j=1, \ldots, k-d)\right\}$. We justify the following observations.

(A) By (2.4), for each $y_{i},\left[y_{i}, w\right],\left[y_{i}, z\right] \notin A(D)$.

(B) The set $\left\{y_{1}, y_{2}, \cdots, y_{d}\right\}$ must be an independent set. This is warranted by (2.4).

(C) Suppose that Lemma 2.2.5(i) or (ii) does not hold. Then $d \leq k-1$. 
In fact, if $d=k$, then $M=M^{\prime}$ and each $x_{i}$ is adjacent to both $w$ and $z$. By (2.3), for each $e_{i}=\left[x_{i}, y_{i}\right] \in M$, we must have $\left(x_{i}, w\right),\left(x_{i}, z\right),\left(w, x_{i}\right),\left(z, x_{i}\right) \in A(D)$. Hence Lemma 2.2.5(i) must hold. Furthermore, by Observations (A) and (B), each $y_{i}$ can only be adjacent to $\left\{x_{1}, x_{2}, \cdots, x_{d}\right\}$. By $(2.2)$, for any $i$, we must have $d^{+}\left(y_{i}\right)=d^{-}\left(y_{i}\right)=k$, and for any $1 \leq i, i^{\prime} \leq k$, we must have $\left(x_{i}, y_{i^{\prime}}\right),\left(y_{i^{\prime}}, x_{i}\right) \in A(D)$. Hence Lemma 2.2.5(ii) holds as well.

(D) From Observation (A), for each $e=\left[x_{i}, y_{i}\right] \in M^{\prime},\left|\left(\left\{x_{i}, y_{i}\right\},\{w, z\}\right)_{D} \cup\left(\{w, z\},\left\{x_{i}, y_{i}\right\}\right)_{D}\right| \leq$ 4 .

(E) For each $j$ with $1 \leq j \leq k-d$, there exists exactly one vertex in $\{w, z\}$ which is adjacent to both $r_{j}$ and $s_{j}$.

By the definition of $M^{\prime \prime}$, for each $j$ with $1 \leq j \leq k-d$, there exists at most one vertex in $\{w, z\}$ which is adjacent to both $r_{j}$ and $s_{j}$. By contradiction, we may assume by symmetry that $\left[r_{1}, s_{1}\right] \in M^{\prime \prime}$ with $\left[z, s_{1}\right] \notin A(D)$ and $\left[w, s_{1}\right] \notin A(D)$. Then $\mid\left(\{w, z\},\left\{r_{1}, s_{1}\right\}\right)_{D} \cup$ $\left(\left\{r_{1}, s_{1}\right\},\{w, z\}\right)_{D} \mid \leq 2$. For any other $\left[r_{j}, s_{j}\right] \in M^{\prime \prime}$ with $j \geq 2$, we have $\mid\left(\{w, z\},\left\{r_{j}, s_{j}\right\}\right)_{D} \cup$ $\left(\left\{r_{j}, s_{j}\right\},\{w, z\}\right)_{D} \mid \leq 4$. It follows from (2.3) and Observation (D) that

$$
\begin{aligned}
4 k= & \left|(\{w, z\}, V(M))_{D} \cup(V(M),\{w, z\})_{D}\right| \\
= & \left|\left(\{w, z\}, V\left(M^{\prime}\right)\right)_{D} \cup\left(V\left(M^{\prime}\right),\{w, z\}\right)_{D}\right|+\left|\left(\{w, z\},\left\{r_{1}, s_{1}\right\}\right)_{D} \cup\left(\left\{r_{1}, s_{1}\right\},\{w, z\}\right)_{D}\right| \\
& +\sum_{j=2}^{k-d}\left|\left(\{w, z\},\left\{r_{j}, s_{j}\right\}\right)_{D} \cup\left(\left\{r_{j}, s_{j}\right\},\{w, z\}\right)_{D}\right| \leq 4(k-1)+2<4 k,
\end{aligned}
$$

a contradiction. This justifies Observation (E).

(F) For any $\left[x_{i}, y_{i}\right] \in M^{\prime}$ and for any $\left[r_{j}, s_{j}\right] \in M^{\prime \prime},\left[y_{i}, r_{j}\right],\left[y_{i}, s_{j}\right] \notin A(D)$. In fact, if $\left[y_{i}, r_{j}\right] \in$ $A(D)$, then by Observation (E), we may assume that $\left[r_{j}, s_{j}\right] \in M_{w}$, and so $\left\{\left[w, s_{j}\right],\left[r_{j}, s_{j}\right],\left[r_{j}, y_{i}\right]\right.$, $\left.\left[x_{i}, y_{i}\right],\left[z, x_{i}\right]\right\}$ will induce an $M$-augmenting path, contrary to (2.4) (same argument when $\left.\left[y_{i}, s_{j}\right] \in A(D)\right)$. This justifies $(\mathrm{F})$.

We argue by contradiction to prove (i) and (ii). As $M^{\prime}=M_{w} \cap M_{z} \neq \emptyset, d \geq 1$ and so $y_{1}$ exists. By Observations (A), (B) and (F), $y_{1}$ can only be adjacent to $\left\{x_{1}, x_{2}, \cdots, x_{d}\right\}$. Hence $d\left(y_{1}\right) \leq 2\left|\left\{x_{1}, x_{2}, \cdots, x_{d}\right\}\right|=2 d$. By (2.2) and by Observation (C), that $2 k \leq d\left(y_{1}\right) \leq 2 d \leq$ $2(k-1)$, a contradiction. This implies that we must have $d=k$, and so by Observation (C), this proves the lemma for Case 2 .

\subsection{Sharpness of the Main Result}

We show by the following examples that the main result is sharp: 
example 1. Let $D_{t}, t \geq 1$ be the digraphs whose underlying undirected graphs are $K_{2, t+2},(t \geq 1)$ where $V\left(D_{t}\right)=\{a, b\} \cup\left(\cup_{i=1}^{t+2}\left\{v_{i}\right\}\right)$ and $A\left(D_{t}\right)=\left\{\left(a, v_{1}\right),\left(v_{1}, b\right)\right\} \cup\left(\cup_{j=2}^{t+2}\left\{\left(v_{j}, a\right),\left(b, v_{j}\right)\right\}\right)$.(see Figure 2.1). In this infinite family of digraphs $\lambda\left(D_{t}\right)=1$ and $\alpha^{\prime}\left(D_{t}\right)=2 \forall t \geq 1$, but $D_{t}$ is not supereulerian.

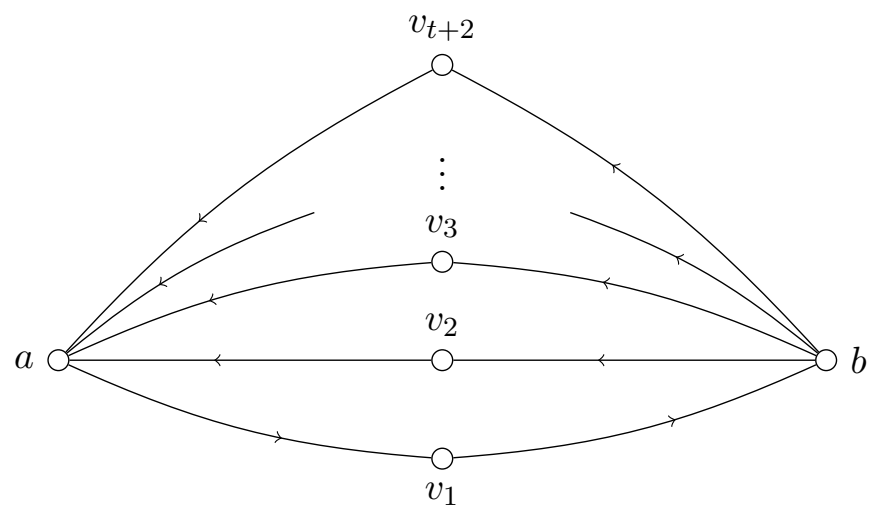

Figure 2.1. The digraph $D_{t}$.

example 2. Let $D_{1} \cong K_{2}^{*}$ and $D_{2} \cong K_{2}^{*}$ be two vertex disjoint complete digraphs with $V\left(D_{1}\right)=\left\{a_{1}, a_{2}\right\}$ and $V\left(D_{2}\right)=\left\{b_{1}, b_{2}\right\}$. Let $U$ be a set of independent vertices disjoint from $V\left(D_{1}\right) \cup V\left(D_{2}\right)$ with $|U|=3$. Let $D$ be the digraph obtained from the disjoint union $D_{1} \cup$ $D_{2} \cup U$ by adding all arcs directed from every vertex in $U$ and $D_{2}$ to every vertex in $D_{1}$, and all arcs directed from every vertex in $D_{2}$ to every vertex in $U$, and then by adding the $\operatorname{arcs}$ $\left\{\left(a_{1}, b_{1}\right),\left(a_{2}, b_{2}\right)\right\} . D$ is not supereulerian. This can be seen by letting $A=\left\{a_{1}, a_{2}\right\}$. By (1.1) in definition 1.2.14, direct computation yields that $\left|\partial_{D}^{+}(A)\right|=2,\left|(U, B)_{D}\right|=0$ and $\tau(U)=3$. Thus $h(U)<0$, and so by Proposition 1.2.15, $D$ is not supereulerian eventhough $\lambda(D)=2$ and $\alpha^{\prime}(D)=3$.

\subsection{Proof of the Main Result}

Throughout this section, $D$ denotes a digraph and $k \geq 1$ be an integer. In this section, we shall prove a slightly stronger version than Theorem 2.1.2, stated as Theorem 2.4.1 below. By (2.1), Theorem 2.1.2 follows immediately from Theorem 2.4.1(i). As a byproduct in the argument, we also prove that if for every vertex $v \in V(D), \min \left\{d^{+}(v), d^{-}(v)\right\} \geq \alpha^{\prime}(D)>0$, then $\lambda(D) \geq \alpha^{\prime}(D)$, as stated in Theorem 2.4.1(ii) below.

Theorem 2.4.1. Let $k>0$ be an integer, $D$ be a digraph on $n \geq 2 k$ vertices with $\alpha^{\prime}(D)=k$. 
Suppose that if $n \leq 2 k+2$, then $D$ is strong. If

$$
\min \left\{d^{+}(v), d^{-}(v)\right\} \geq k, \text { for every vertex } v \in V(D)
$$

then each of the following holds.

(i) $D$ is supereulerian.

(ii) $\lambda(D) \geq k$.

Proof Let $M$ be a matching of maximum size of $D$. We proceed our proof in the following cases.

Case 1. $2 k \leq n \leq 2 k+1$.

If $n=2 k$, then by theorem $2.2 .2, D$ is hamiltonian, and so $D$ is also supereulerian. If $n=2 k+1$, then by Theorem $1.2 .12, D$ is also supereulerian. It remains to prove that $\lambda(D) \geq k$.

Let $X$ be an arbitrary nonempty proper subset of $V(D)$, and let $Y=V(D)-X$. Since $|X|+|Y|=n \leq 2 k+1$, either $1 \leq|X| \leq k$ or $1 \leq|Y| \leq k$. By symmetry, we may assume that $1 \leq|X|=m \leq k$. By (2.7), for each $x \in X . \quad\left|(\{x\}, Y)_{D}\right| \geq k-(m-1)$. Thus $\left|\partial_{D}^{+}(X)\right| \geq m(k-(m-1))=-m^{2}+m(k+1)$. As this is a quadratic function with $1 \leq m \leq k$, it follows that $\left|\partial_{D}^{+}(X)\right| \geq-m^{2}+m(k+1) \geq k$, and so by $(2.1), \lambda(D) \geq k$. This proves Case 1 .

Case 2. $n \geq 2 k+2$.

(i). Since $k \geq 1$, by (2.7), $D$ must has an eulerian subdigraph. Let $S$ be an eulerian subdigraph of $D$ with

$$
|V(S)| \text { is maximized among all eulerian subdigraphs of } D \text {. }
$$

Let $s=|V(S)|$. If $s=n$, then $S$ is a spanning eulerian subdigraph of $D$ and we are done. By contradiction, we assume that $n>s \geq 2$. Hence $V(D)-V(S) \neq \emptyset$. We are to prove (i) in the following two subcases.

Subcase 2.1. $(V(D)-V(S))-V(M) \neq \emptyset$.

Pick $v \in(V(D)-V(S))-V(M)$. Since $A(S) \neq \emptyset$, we pick an arc $e=[x, y] \in A(S)$. Since $M$ is a maximum matching of $D, V(M) \cap\{x, y\} \neq \emptyset$, and so we may assume that $x \in V(M)$. Therefore, there exists an $\operatorname{arc} a=[x, z] \in M$. If $x, z \in V(S)$, then by Lemma 2.2.5(i), there exists a vertex $v(a) \in\{x, z\}$ such that $(v, v(a)),(v(a), v) \in A(D)$. It follows that $A(S) \cup$ $\{(v, v(a)),(v(a), v)\}$ induces an eulerian subdigraph $S_{1}$ with $\left|V\left(S_{1}\right)\right|>|V(S)|$, contrary to (2.8). Hence we may assume that $z \notin V(S)$. By Lemma 2.2.5(ii), we have $(x, z),(z, x) \in A(D)$, and 
so $A(S) \cup\{(x, z),(z, x)\}$ induces an eulerian subdigraph $S_{2}$ with $\left|V\left(S_{2}\right)\right|>|V(S)|$, contrary to (2.8) also. This completes the proof for Subcase 2.1 .

Subcase 2.2. $V(D)-V(S) \subseteq V(M)$.

Pick $v \in V(D)-V(S)$. Since $v \in V(M)$, there must be an arc $a=[u, v] \in M$. If $u \in V(S)$, then by Lemma 2.2.5(ii), both $(u, v),(v, u) \in A(D)$. Hence $A(S) \cup\{(u, v),(v, u)\}$ induces an eulerian subdigraph $S_{3}$ of $D$ with $\left|V\left(S_{3}\right)\right|>|V(S)|$, contrary to (2.8). Therefore, we must have $u \notin V(S)$.

Since $n \geq 2 k+2=|V(M)|+2$, there must be a vertex $w \in V(D)-V(M)$. Since $V(D)-V(S) \subseteq V(M), w \in V(S)$. By Lemma 2.2.5(i), there must be a $v(a) \in\{u, v\}$ such that $(w, v(a)),(v(a), w) \in A(D)$. It follows that $A(S) \cup\{(w, v(a)),(v(a), w)\}$ induces an eulerian subdigraph $S_{4}$ of $D$ with $\left|V\left(S_{4}\right)\right|>|V(S)|$, contrary to (2.8). This completes the proof of Theorem 3.1.1(i).

(ii). Let $X$ satisfying $\emptyset \neq X \subset V(D)$ be an arbitrary nonempty proper vertex subset. We are to prove $\left|\partial_{D}^{+}(X)\right| \geq k$.

Let $Z=V(D)-V(M)$. By Lemma 2.2.5 (i), for any $e=[u, v] \in M$, there exists a unique $v(e) \in\{u, v\}$ such that for any $z \in Z,(z, v(e)),(v(e), z) \in A(D)$. Let $M_{v}=\{v(e): e \in M\}$, and $M_{u}=V(M)-M_{v}$. Let $m \geq 2$ be the integer satisfying $n=2 k+m$. By Lemma 2.2.5, for each $v \in M_{v}$, and for any $u \in Z \cup M_{u},(v, u),(u, v) \in A(D)$.

We consider the following cases.

Case 1. $\left(M_{u} \cup Z\right) \subseteq X$ (or $\left.\left(M_{u} \cup Z\right) \cap X=\emptyset\right)$. We assume that $M_{u} \cup Z \subseteq X$ as by symmetry, the proof for $\left(M_{u} \cup Z\right) \cap X=\emptyset$ is similar. As $V(D)=M_{u} \cup M_{v} \cup Z$, there exists a $y \in M_{v}-X \subseteq V(D)-X$. By Lemma 2.2.5, $\left|\partial_{D}^{+}(X)\right| \geq\left|\left(M_{u} \cup Z,\{y\}\right)_{D}\right|=|Z|+\left|M_{u}\right|=k+m>k$.

Case 2. $M_{v} \subseteq X$ (or $M_{v} \subseteq V(D)-X$ ). We assume that $M_{v} \subseteq X$, as by symmetry, the proof for $M_{v} \subseteq V(D)-X$ is similar. Then $M_{u} \cup Z-X \neq \emptyset$. Pick $y \in M_{u} \cup Z-X$. Then by Lemma 2.2.5, $\left|\partial_{D}^{+}(X)\right| \geq\left|\left(M_{v},\{y\}\right)_{D}\right|=\left|M_{v}\right|=k$.

Case 3. Both $M_{u} \cup Z-X \neq \emptyset$ and $X \cap\left(M_{u} \cup Z\right) \neq \emptyset$, and both $M_{v}-X \neq \emptyset$ and $X \cap M_{v} \neq \emptyset$.

Pick $x \in X \cap\left(M_{u} \cup Z\right)$ and $y \in\left(M_{u} \cup Z\right)-X$. Then by Lemma 2.2.5,

$$
\left|\partial_{D}^{+}(X)\right| \geq\left|\left(\{x\}, M_{v}-X\right)_{D}\right|+\left|\left(M_{v} \cap X,\{y\}\right)_{D}\right|=\left|M_{v}-X\right|+\left|M_{v} \cap X\right|=\left|M_{v}\right|=k .
$$

It follows that we always have $\left|\partial_{D}^{+}(X)\right| \geq k$, and so $\lambda(D) \geq k$. This proves (ii). 


\section{Chapter 3}

\section{Locally Dense Supereulerian Digraphs}

\subsection{Introduction}

We consider finite graphs and finite and simple digraphs. Usually, we use G to denote a graph and $\mathrm{D}$ to denote a digraph. Undefined terms and notations will follow [8] for graphs and [4] for digraphs. For a digraph $D$, let $G(D)$ denote underlying undirected graph of $D$, obtained from $D$ by erasing the directions on each arcs of $D$. In particular, $\lambda(D)$ denotes the arc-strong connectivity of a digraph $\mathrm{D}, c(D)$ denotes the number of components of $G(D)$. We use the notation $(u, v)$ to denote an arc oriented from $u$ to $v$ in a digraph; For graphs $H$ and $G$, by $H \subseteq G$ we mean that $H$ is a subgraph of $G$. Similarly, for digraphs $H$ and $D$, by $H \subseteq D$ we mean that $H$ is a subdigraph of $D$. If $X \subseteq V(D) \cup A(D)$, then $D[X]$ denotes the subdigraph induced by $X$. For a subdigraph $S$ of a digraph $D$, and for subsets $X \subseteq A(S)$ and $Y \subseteq A(D)-A(S)$, we use $S-X+Y$ to denote $D[(A(S)-X) \cup Y]$, the subdigraph induced by the arc subset $(A(S)-X) \cup Y$.

A digraph $D$ is strong if $\lambda(D)>0$. For $X, Y \subseteq V(D)$, define

$$
(X, Y)_{D}=\{(x, y) \in A(D): x \in X, y \in Y\}
$$

and

$$
\partial_{D}^{+}(X)=(X, V(D)-X)_{D} \text { and } \partial_{D}^{-}(X)=(V(D)-X, X)_{D}
$$

For any vertex $v \in V(D)$, we define the out neighbors of $v$ by $N^{+}(v)=\{u: u \in V(D)$ and $(v, u) \in A(D)\}$, the in neighbors of $v$ by $N^{-}(v)=\{u: u \in V(D)$ and $(u, v) \in A(D)\}$ and 
the neighbors of $v$ by $N(v)=N^{+}(v) \cup N^{-}(v)$. Accordingly, $d_{D}^{+}(v)=\left|\partial_{D}^{+}(\{v\})\right|$ and $d_{D}^{-}(v)=$ $\left|\partial_{D}^{-}(\{v\})\right|$ are the out-degree and the in-degree of $v$ in $D$, respectively. Let

$$
\partial_{D}(\{v\})=\partial_{D}^{+}(\{v\}) \cup \partial_{D}^{-}(\{v\}) \text {, and } d_{D}(v)=d_{D}^{+}(v)+d_{D}^{-}(v) .
$$

When the digraph $D$ is understood from the context, we often omit the subscript $D$. By the definition of $\lambda(D)$ in [4], we note that for any integer $k \geq 0$ and a digraph $D$,

$$
\lambda(D) \geq k \text { if and only if for any } \emptyset \neq X \subset V(D),\left|\partial_{D}^{+}(X)\right| \geq k \text {. }
$$

Following the definition in [4] (Page 11), for a subdigraph $H$ of a digraph $D$, an $(x, y)$-dipath $P$ is an $(H, H)$-dipath if $x, y \in V(H)$ and $V(P) \cap V(H)=\{x, y\}$.

A graph $G$ is locally connected if for every vertex $v \in V(G)$, the vertices adjacent to $v$ induce a connected subgraph in $G$. A vertex $v \in V(D)$ is $k^{+}$-locally-arc-connected, (or $k^{-}$-locally-arcconnected, or $k$-locally-arc-connected, respectively) if $\lambda\left(D\left[N^{+}(v)\right]\right) \geq k\left(\lambda\left(D\left[N^{-}(v)\right]\right) \geq k\right.$, or $\lambda(D[N(v)]) \geq k$, respectively). A digraph $D$ is $k^{+}$-locally-arc-connected, (or $k^{-}$-locallyarc-connected, or $k$-locally-arc-connected, respectively) if every vertex of $D$ is $k^{+}$-locally-arcconnected, (or $k^{-}$-locally-arc-connected, or $k$-locally-arc-connected, respectively).

It is well known (for example, Corollary 1 of [10]) that every connected, locally-connected graph other than $K_{2}$ is supereulerian. The problem investigated in this chapter is whether strong and locally strong digraphs will behave similarly, and how local structure in digraph will warrant the existence of a spanning eulerian subdigraph. In the next section, we shall show that for any integer $k>0$, there exists an infinite family of strong, $k^{+}$-locally-arc-connected non-supereulerian digraphs which is also an infinite family of strong, $k$-locally-arc-connected non-supereulerian digraphs. These suggest that local connectivity may not be sufficient for supereulerian digraphs. This motivates us to seek whether locally dense conditions for supereulerian digraphs.

For rational numbers $\alpha$ and $\beta$, define a strong digraph $D$ to be locally $(\alpha, \beta)^{+}$-dense if $\forall v \in V(D), d_{D\left[N^{+}(v)\right]}(u) \geq \alpha\left|N^{+}(v)\right|+\beta, \forall u \in N^{+}(v)$; and $D$ is locally $(\alpha, \beta)^{+}$-arc-connected if $\forall v \in V(D), \lambda\left(D\left[N^{+}(v)\right]\right) \geq \alpha\left|N^{+}(v)\right|+\beta$; as well as ; $D$ is locally $(\alpha, \beta)$-dense if $\forall v \in$ $V(D), d_{D[N(v)]}(u) \geq \alpha|N(v)|+\beta, \forall u \in N(v)$; and $D$ is locally $(\alpha, \beta)$-arc-connected if $\forall v \in$ $V(D), \lambda(D[N(v)]) \geq \alpha|N(v)|+\beta$. In Section 3, we will prove the following main results in this chapter.

Theorem 3.1.1. Every locally $\left(\frac{4}{3}, \frac{-7}{3}\right)^{+}$-dense strong simple digraph is supereulerian.

Corollary 3.1.2. Every locally $\left(\frac{2}{3}, 0\right)^{+}$-arc-connected strong simple digraph is supereulerian. 
In the next section, we will present examples to show that there exist infinitely many strong, locally strong digraphs may not be supereulerian. We will also present examples to show that for some fractional numbers $\alpha$ and $\beta$, there exist strong simple nonsupereulerian digraphs $D$ satisfying the condition that for any $v \in V(D)$, it holds that $d_{D\left[N^{+}(v)\right]}(u) \geq \alpha\left|N^{+}(v)\right|+\beta-1$, $\forall u \in N^{+}(v)$. This shows that the main result Theorem 3.1.1 is best possible in some sense.

\subsection{Examples}

In this section, we shall first show that for any integer $k>0$, a strong, $k^{+}$-locally-arc-connected digraph and a strong, $k$-locally-arc-connected digraph may not be supereulerian. For this purpose, we shall display a necessary condition for supereulerian digraphs, established in [16].

Let $D$ be a strong digraph and $U \subset V(D)$. Then in $D[U]$, the digraph induced by $U$, we can find some ditrails $P_{1}, \ldots, P_{t}$ such that $\bigcup_{i=1}^{t} V\left(P_{i}\right)=U$ and $A\left(P_{i}\right) \cap A\left(P_{j}\right)=\emptyset$ for any $i \neq j$. Let $\tau(U)$ be the minimum value of such $t$. Then $c(G(D[U])) \leq \tau(U) \leq|U|$, where $c(G(D[U]))$ is the number of components of the underlying graph of $D[U]$. For any $A \subseteq V(D)-U$, denote $B:=V(D)-U-A$ and let

$$
\begin{aligned}
h(U, A): & =\min \left\{\left|\partial_{D}^{+}(A)\right|,\left|\partial_{D}^{-}(A)\right|\right\}+\min \left\{\left|(U, B)_{D}\right|,\left|(B, U)_{D}\right|\right\}-\tau(U), \text { and } \\
h(U): & =\min \{h(U, A): A \cap U=\emptyset\} .
\end{aligned}
$$

In [16], Hong et al give the following proposition, and use it to find some classes of digraphs which are not supereulerian.

Proposition 3.2.1. (Proposition (2.1) of Hong et al [16]) If D has a spanning eulerian subdigraph, then for any $U \subset V(D), h(U) \geq 0$.

This proposition can be applied to show that there exists a family of strong and locally $k^{+}-$ arc-connected non-supereulerian digraphs which is also locally $k$-arc-connected non-supereulerian digraphs.

Example 3.2.2. Let $k>0, l>(k+1)^{2}$ and $n_{1} \geq n_{2} \geq k+2$ be integers, $D_{1}$ and $D_{2}$ be two vertex disjoint complete digraphs on $n_{1}$ and $n_{2}$ vertices, respectively, $X \subset V\left(D_{1}\right)$ and $Y \subset V\left(D_{2}\right)$ with $|X|=|Y|=k+1$ and let $U$ be a set of independent vertices disjoint from $V\left(D_{1}\right) \cup V\left(D_{2}\right)$ with $|U|=l$. Let $\mathcal{D}(k, l)$ denote the family of digraphs such that $D \in \mathcal{D}(k, l)$ if and only if $D$ is the digraph obtained from the disjoint union $D_{1} \cup D_{2} \cup U$ by adding all arcs directed from every vertex in $U$ and $D_{2}$ to every vertex in $D_{1}$, and all arcs directed from every vertex in $D_{2}$ to every 
vertex in $U$, and then by adding $(k+1)^{2}$ arcs from $X$ to $Y$. (See Figure 3.1). We shall justify that $D$ is a locally $k^{+}$-arc-connected digraph below. Let $A=V\left(D_{1}\right)$. Then

$$
h(U, A)=\left|\partial_{D}^{+}(A)\right|+\left|(U, V(D)-U-A)_{D}\right|-\tau(U)=(k+1)^{2}+0-l<0 .
$$

By Proposition 3.2.1, $D$ is not supereulerian. $D$ is strong because for any two vertices $v, z \in$ $V(D)$, there exists a $(v, z)$-dipath.

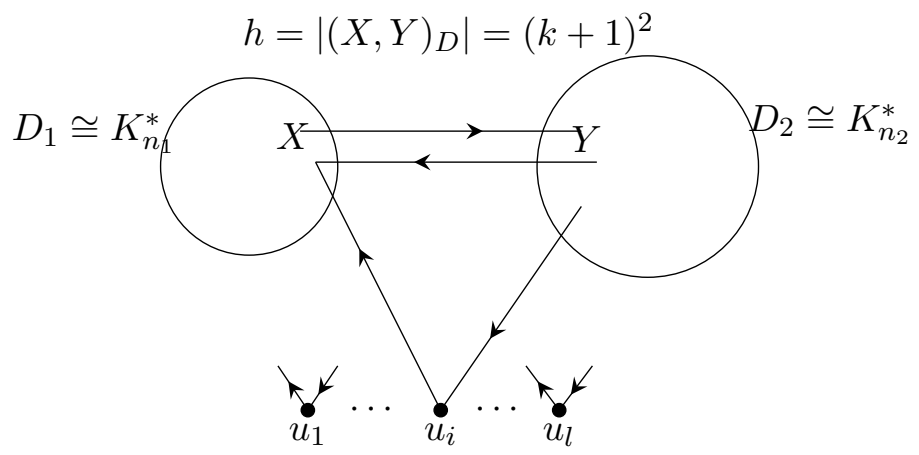

Figure 3.1. The digraph $D=D\left(n_{1}, n_{2}, l\right)$, with $n_{1}, n_{2} \geq k+2$, and $l>(k+1)^{2}$.

Let $h=(k+1)^{2}$. We will verify our claims above that $D$ is both a locally $k^{+}$-arc-connected digraph and a locally $k$-arc-connected digraph. The observations below follow from the definition of $D$.

Observation 3.2.3. Let $V_{1}=V\left(D_{1}\right)$ and $V_{2}=V\left(D_{2}\right)$.

(i) $\left|\left(V_{1}, V_{2}\right)_{D}\right|=(k+1)^{2}$ and $\left|\left(V_{2}, V_{1}\right)_{D}\right|=n_{1} n_{2} \geq(k+2)^{2}$.

(ii) $\left|\left(V_{1}, U\right)_{D}\right|=0$ and $\left|\left(U, V_{1}\right)_{D}\right|=\ln _{1}$.

(iii) $\left|\left(V_{2}, U\right)_{D}\right|=\ln _{2}$ and $\left|\left(U, V_{2}\right)_{D}\right|=0$.

(iv) If $v \in V_{2}$, then $N^{+}(v)=V(D)-\{v\}$.

(v) If $v \in V_{1} \cup U-X$, then $N^{+}(v)=V_{1}-\{v\}$; if $v \in X$, then $N^{+}(v)=V_{1} \cup Y-\{v\}$.

(vi) If $v \in V_{1} \cup V_{2}$, then $N(v)=V(D)-\{v\}$; if $v \in U$, then $N(v)=V_{1} \cup V_{2}$.

(vii) If two vertices $r$ and $s$ are contained in a complete subdigraph $K$ with $|V(K)| \geq k+1$, then $K$ has $k$ arc-disjoint $(r, s)$-dipaths.

Claim 1. For any $v \in V(D)$, and for any two vertices $r, s \in V(D)-\{v\}, D-\{v\}$ has $k$ arc-disjoint $(r, s)$-dipaths.

Assume first that $r \in V_{1} \cup U$. Since $D_{1} \cong K_{n_{1}}^{*}$ with $n_{1} \geq k+2$, it follows by Observation 3.2.3(vii) that $\left|\{r, s\} \cap V_{1}\right| \leq 1$. If $|\{r, s, v\} \cap X| \leq 1$, then since $|X|=|Y|=k+1$, there exist distinct vertices $x_{1}, x_{2}, \ldots, x_{k} \in X-\{r, s, v\}$ and $y_{1}, y_{2}, \ldots, y_{k} \in Y-\{v\}$ such that if $s \in Y$, then 
$y_{k}=s$. Since $\left|(X, Y)_{D}\right|=(k+1)^{2}$ and by Observation 3.2.3(i)-(iii), if $y_{k} \neq s$, then the dipaths $r x_{i} y_{i} s,(1 \leq i \leq k)$, are $k$ arc-disjoint $(r, s)$-dipaths in $D-\{v\}$; if $y_{k}=s$, then the dipaths $r x_{i} s,(1 \leq i \leq k)$, are $k$ arc-disjoint $(r, s)$-dipaths in $D-\{v\}$. Since $\left|(X, Y)_{D}\right|=(k+1)^{2}$ and by Observation 3.2.3(i),(iii),(vii), if $r, v \in X$, then for distinct vertices $y_{1}, y_{2}, \ldots, y_{k} \in Y-\{s\}$, $r y_{i} s$, are $k$ arc-disjoint $(r, s)$-dipaths in $D-\{v\}$. Since $\left|V_{1}\right| \geq k+2$, there exist distinct vertices $h_{1}, h_{2}, \ldots, h_{k} \in V_{1}-\{r, s, v\}$. Thus by Observation 3.2.3 (ii),(vii), if $s, v \in X, r h_{i} s,(1 \leq i \leq k)$, are $k$ arc-disjoint $(r, s)$-dipaths in $D-\{v\}$.

Therefore, we assume that $r \in V_{2}$. Since $D_{2} \cong K_{n_{2}}^{*}$ with $n_{2} \geq k+2$, it follows by Observation 3.2 .3 (vii) that $s \in V(D)-V_{2}$. Since $\left|V_{2}\right| \geqslant k+2$, there exist distinct vertices $d_{1}, d_{2}, \ldots, d_{k} \in$ $V_{2}-\{r, v\}$. Thus by Observation 3.2.3(i), the dipaths $r d_{i} s$, are $k$ arc-disjoint $(r, s)$-dipaths in $D-\{v\}$. This proves Claim 1 .

Claim 2. D is locally $k^{+}$-arc-connected.

We shall show that for any vertex $v \in V(D)$, and for any two vertices $r, s \in N^{+}(v), D^{[}\left[N^{+}(v)\right]$ contains $k$ arc-disjoint $(r, s)$-dipaths. By Observation 3.2.3(vii), we may assume that

$$
D\left[N^{+}(v)\right] \text { has no complete subdigraphs } K \text { with }|V(K)| \geq k+1 \text { and } r, s \in V(K) .
$$

By Observation 3.2.3(v), if $v \in V_{2}$, then $N^{+}(v)=V(D)-\{v\}$. By Claim 1, there are $k$ arc-disjoint $(r, s)$-dipaths in $D\left[N^{+}(v)\right]$. Hence we may assume that $v \in V_{1} \cup U$. By Observation 3.2.3(v), if $v \in V_{1} \cup U-X$, then $N^{+}(v)=V_{1}-\{v\}$; if $v \in X$, then $N^{+}(v)=V_{1} \cup Y-\{v\}$.

Therefore by (3.3), we may assume that $v \in X, N^{+}(v)=V_{1} \cup Y-\{v\}$. As $\left|(X, Y)_{D}\right|=$

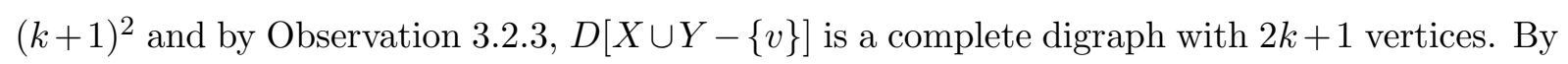
(3.3), we must have $\left|\left(V_{1}-X\right) \cap\{r, s\}\right|=|Y \cap\{r, s\}|=1$ and $X \cap\{r, s\}=\emptyset$. Since $|X|=k+1$, $\left|(X, Y)_{D}\right|=(k+1)^{2}$ and since $X \cap\{r, s\}=\emptyset$, we have $r \in\left(V_{1}-X\right) \cup Y$ and there exist distinct vertices $x_{1}, x_{2}, \ldots, x_{k} \in X-\{v\}$ such that $r x_{i} s, 1 \leq i \leq k$, are $k$ arc-disjoint $(r, s)$-dipaths in $D\left[N^{+}(v)\right]$. This proves Claim 2 .

Claim 3. $D$ is locally $k$-arc-connected.

Again we shall show that for any vertex $v \in V(D)$, and for any two vertices $r, s \in N(v)$, $D[N(v)]$ contains $k$ arc-disjoint $(r, s)$-dipaths. By Observation 3.2.3(vii), we may assume that

$D[N(v)]$ has no complete subdigraphs $K$ with $|V(K)| \geq k+1$ and $r, s \in V(K)$.

If $v \in V_{1} \cup V_{2}$, then by Observation 3.2.3(vi), $N(v)=V(D)-\{v\}$, and so by Claim 1, there exist $k$ arc-disjoint $(r, s)$-dipaths in $D[N(v)]$. Hence we only need to show Claim 3 when $v \in U$. By Observation 3.2.3(vi), $N(v)=V_{1} \cup V_{2}$. 
By (3.4), $\left|V_{1} \cap\{r, s\}\right|=\left|V_{2} \cap\{r, s\}\right|=1$. Since $|X|=|Y|=k+1$, there exist distinct vertices $x_{1}, x_{2}, \ldots, x_{k} \in X-\{r, s\}$ and $y_{1}, y_{2}, \ldots, y_{k} \in Y-\{r, s\}$. Since $\left|(X, Y)_{D}\right|=(k+1)^{2}$ and by Observation 3.2.3(i)-(iii), the dipaths $r x_{i} y_{i} s,(1 \leq i \leq k)$, are $k$ arc-disjoint $(r, s)$-dipaths in $D[N(v)]$. This justifies Claim 3 .

Remark 3.2.4. By Claims 2 and 3, we conclude that any $D=D\left(n_{1}, n_{2}, l\right)$ with $n_{1} \geq n_{2} \geq$ $k+2$ and $l>(k+1)^{2}$ is locally $k^{+}$-arc-connected and locally $k$-arc-connected, but it is not supereulerian. Moreover, by Claim 1, D is k-arc-connected which means that the following well known theorem due to Jaeger and Catlin cannot be extended to digraphs.

Theorem 3.2.5. ([10]) Every 4-edge-connected graph is supereulerian.

Next, we will present an example to address the sharpness of Theorem 3.1.1.

Example 3.2.6. We will first present a building block digraph, and then we will use the building block to build an infinite family of digraphs to show that Theorem 3.1.1 is best possible in some sense.

(i) Let $L$ be a digraph with $V(L)=\left\{v_{1}, v_{2}, v_{3}, v_{4}, v_{5}, v_{6}\right\}$ and $A(L)=\left\{\left(v_{1}, v_{2}\right),\left(v_{2}, v_{1}\right),\left(v_{1}, v_{4}\right),\left(v_{3}, v_{2}\right)\right.$, $\left.\left(v_{2}, v_{4}\right),\left(v_{3}, v_{1}\right),\left(v_{4}, v_{3}\right),\left(v_{3}, v_{6}\right),\left(v_{3}, v_{5}\right),\left(v_{6}, v_{4}\right),\left(v_{5}, v_{4}\right),\left(v_{5}, v_{6}\right),\left(v_{6}, v_{5}\right)\right\}$ as depicted in Figure 3.2.

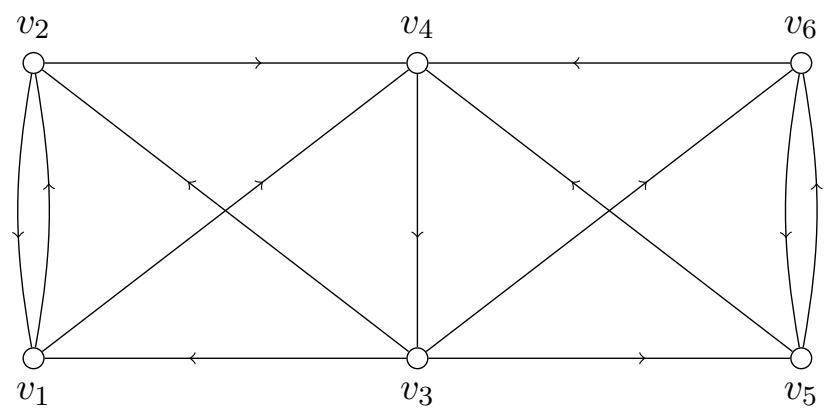

Figure 3.2.

(ii) Using the notation in Figure 3.2, we can fix the vertex set of one of the two $K_{2}^{*}$ 's in $L$, $L\left[\left\{v_{1}, v_{2}\right\}\right]$ and $L\left[\left\{v_{5}, v_{6}\right\}\right]$, and define it as a distinguished pair. Let $\{a, b\}$ be a distinguished pair of $L$, and denote $L$ by $L(a, b)$. Let $L_{1}\left(a^{\prime}, b^{\prime}\right)$ and $L_{2}\left(a^{\prime \prime}, b^{\prime \prime}\right)$ be two copies of $L\left(v_{1}, v_{2}\right)$. Obtain a new digraph $L_{1} \oplus L_{2}$ from $L_{1}$ and $L_{2}$ by identifying $a^{\prime}$ and $a^{\prime \prime}$ to form a new vertex a, and identifying $b^{\prime}$ and $b^{\prime \prime}$ to form a new vertex $b$. See Figure 3.3 for an drawing of $L_{1} \oplus L_{2}$ with $a=v_{1}$ and $b=v_{2}$. 


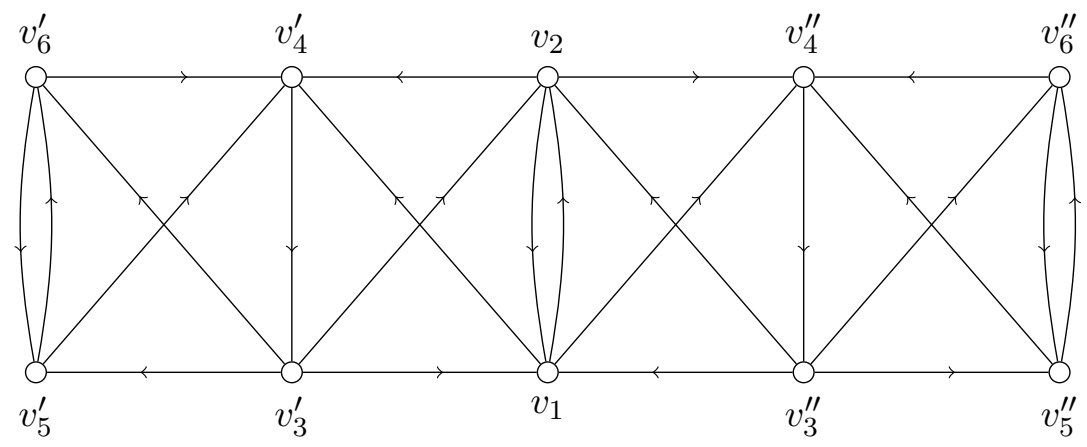

Figure 3.3.

Observation 3.2.7. We make the following observations on the digraph $L$ defined in Example 3.2.6.

(i) $D$ is strong. This can be verified by observing that each of these subdigraphs $D\left[\left\{v_{1}, v_{2}\right\}\right]$, $\left.D\left[v_{2}, v_{4}, v_{3}\right\}\right], D\left[\left\{v_{4}, v_{3}, v_{6}\right\}\right]$ and $D\left[\left\{v_{5}, v_{6}\right\}\right]$ are directed circuits.

(ii) $D$ is not supereulerian. This can be seen by letting $U=\left\{v_{1}, v_{2}, v_{5}, v_{6}\right\}$ and $A=\left\{v_{4}\right\}$. By (3.2), direct computation yields that $\left|\partial_{D}^{+}(A)\right|=1,\left|(U, B)_{D}\right|=0$ and $\tau(U)=2$. Thus $h(U)<0$, and so by Proposition 3.2.1, $D$ is not supereulerian.

(iii) For any $i$ with $i \in\{1,2,4,5,6\}$, direct computation shows that $\forall u \in N^{+}\left(v_{i}\right), d_{D\left[N^{+}\left(v_{i}\right)\right]}(u) \geq$ $\frac{4}{3}\left|N^{+}\left(v_{i}\right)\right|-\frac{7}{3}$.

(iv) For $i=3$, we have $\left|N^{+}\left(v_{3}\right)\right|=4$. For any $z \in N^{+}\left(v_{3}\right)$, we have $d_{D\left[N^{+}\left(v_{3}\right)\right]}(z)=2=$ $\frac{4}{3}\left|N^{+}\left(v_{3}\right)\right|-\frac{7}{3}-1$.

Remark 3.2.8. By Observation 3.2.7, $L$ is a strong nonsupereulerian digraph. Furthermore, for any $v \in V(D)$, it holds that $d_{D\left[N^{+}(v)\right]}(u) \geq \frac{13}{12}\left|N^{+}(v)\right|-\frac{7}{3} \geq \frac{4}{3}\left|N^{+}(v)\right|-\frac{7}{3}-1, \forall u \in N^{+}(v)$. Thus smaller value of $\frac{4}{3}$ or $-\frac{7}{3}$ would not warrant the existence of a spanning ditrail in $D$. To construct an infinity family of such digraphs, we can use the $\oplus$ operation to build large strong but nonsupereulerian digraphs satisfying the same local density conditions. This shows that Theorem 3.1.1 is in some sense, best possible. However, we do not have examples to show that for any sufficiently small real numbers $\epsilon_{1}>0$ and $\epsilon_{2}>0$, there exists strong non-supereulerian digraphs which is locally $\left(\frac{4}{3}-\epsilon_{1}, \frac{-7}{3}\right)^{+}$-dense or locally $\left(\frac{4}{3}, \frac{-7}{3}-\epsilon_{2}\right)^{+}$-dense.

\subsection{Proof of Theorem 3.1.1}

We will present the proof of Theorem 3.1.1 in this section. Assume that

$D$ is a locally $\left(\frac{4}{3}, \frac{-7}{3}\right)^{+}$-dense strong simple digraph. 
Since $D$ is strong, $D$ must have an eulerian subdigraph. Let $S$ be an eulerian subdigraph of $D$ such that among all eulerian subdigraphs of $D$

$$
|V(S)| \text { being maximized. }
$$

If $|V(S)|=|V(D)|$, then $S$ is a spanning eulerian subdigraph of $D$ and we are done. Assume by contradiction that $|V(D)|>|V(S)|>1$. Hence $V(D)-V(S) \neq \emptyset$. Since $D$ is strong and $S$ is a maximum eulerian subdigraph, there exists an $(S, S)$-dipath $Q$ on at least three vertices. Let $Q$ be chosen so that:

the length of a shortest dipath $P$ in $S$ between the endpoints of $Q$ is minimized.

Assume that $V(Q) \cap V(S)=\{v, z\}$ with $(v, x) \in A(Q)$. If $(v, z) \in A(S)$, then $S-(v, z)+A(Q)$ is an eulerian subdigraph with at least one more vertex than $S$, contrary to (3.6). Therefore, there must be a vertex $y \in V(P)-\{v, z\}$ such that $(v, y) \in A(P)$. (See Figure 3.4). Let

$$
\begin{array}{ll} 
& X=N^{+}(v)-(\{x, y\} \cup V(S)) \text { and } Y=N^{+}(v) \cap(V(S)-\{y\}), \\
\text { and } & |X|=k,|Y|=h,|X|+|Y|=m=k+h,\left|N^{+}(v)\right|=n_{v} .
\end{array}
$$

We make the following observations.

Claim 1. Each of the following holds.

(i) For each $x^{\prime} \in X$ with $\left(x, x^{\prime}\right),\left(x^{\prime}, x\right) \in A(D)$, we must have $\left|\left\{\left(y, x^{\prime}\right),\left(x^{\prime}, y\right)\right\} \cap A(D)\right|=0$..

(ii) If $h>0$, then for any $y^{\prime} \in Y$, we cannot have $\left\{\left(y^{\prime}, x\right),\left(x, y^{\prime}\right)\right\} \subset A(D)$.

(iii) $\{(x, y),(y, x)\} \cap A(D)=\emptyset$.

We argue by contradiction to prove Claim 1(i), and assume that for some vertex $x^{\prime} \in X$ with $\left(x, x^{\prime}\right),\left(x^{\prime}, x\right) \in A(D)$, either $\left(y, x^{\prime}\right) \in A(D)$ or $\left(x^{\prime}, y\right) \in A(D)$. If $\left(y, x^{\prime}\right) \in A(D)$, then as $\left.D[(A(Q)-\{v, x)\}) \cup\left\{\left(y, x^{\prime}\right),\left(x^{\prime}, x\right)\right\}\right]$ is a dipath of $D$ intersecting $S$ only at $y, z$, and as the distance from $y$ to $z$ in $S$ is shorter than that from $v$ to $z$ in $S$, a contradiction to (3.7) is obtained. If $\left(x^{\prime}, y\right) \in A(D)$, then $S-(v, y)+\left\{(v, x),\left(x, x^{\prime}\right),\left(x^{\prime}, y\right)\right\}$ is an eulerian subdigraph of $D$ with more vertices than $S$, contrary to (3.6). This proves (i).

We argue by contradiction to prove Claim 1(ii), and assume that for an $y^{\prime} \in Y$, we have $\left\{\left(y^{\prime}, x\right),\left(x, y^{\prime}\right)\right\} \subset A(D)$. Then $S+\left\{\left(x, y^{\prime}\right),\left(y^{\prime}, x\right)\right\}$ is an eulerian subdigraph of $D$ with $|V(S)|+1$ vertices, contrary to (3.6). This justifies Claim 1(ii).

To prove (iii), assume that either $(x, y) \in A(D)$, whence $S-(v, y)+\{(v, x),(x, y)\}$ is an eulerian subdigraph of $D$ with more vertices than $S$, contrary to (3.6); or $(y, x) \in A(D)$, whence (3.7) is violated. Thus (iii) must hold. 


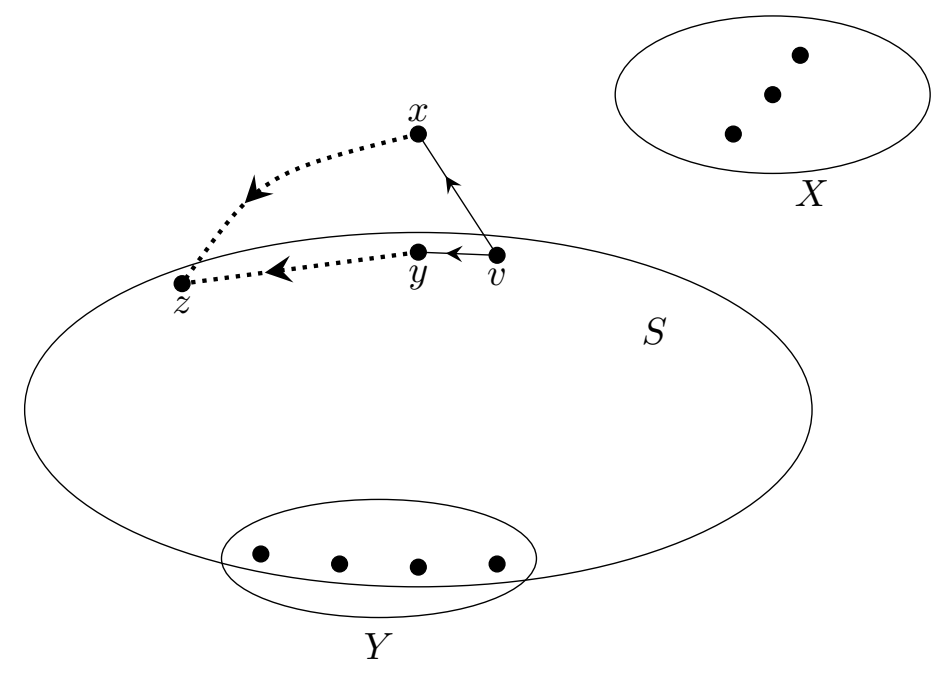

Figure 3.4.

Claim 2. If $d_{D\left[N^{+}(v)\right]}(x) \geq 2 k+h$, then $d_{D\left[N^{+}(v)\right]}(y) \leq 2 h$.

By contradiction, we assume that $d_{D\left[N^{+}(v)\right]}(x) \geq 2 k+h$ and $d_{D\left[N^{+}(v)\right]}(y) \geq 2 h+1$. By Claim 1(ii), $\left|(x, Y)_{D} \cup(Y, x)_{D}\right| \leq h$. Since $d_{D\left[N^{+}(v)\right]}(x) \geq 2 k+h$, for each $x^{\prime} \in X$, we must have both $\left(x, x^{\prime}\right) \cdot\left(x^{\prime} x\right) \in A(D)$. Since $d_{D\left[N^{+}(v)\right]}(y) \geq 2 h+1$ and since $\left|(y, Y)_{D} \cup(Y, y)_{D}\right| \leq 2 h$, there must be an $x^{\prime} \in X$ such that $\left|\left\{\left(x^{\prime}, y\right),\left(y, x^{\prime}\right)\right\} \cap A(D)\right| \geq 1$, contrary to Claim 1(i). Hence Claim 2 must hold.

Claim 3. $m \geq 2$.

If $m=0$, then by (3.8), we have $\left|N^{+}(v)\right|=2$, and so by $(3.5), d_{D\left[N^{+}(v)\right]}(x) \geq \frac{4(2)-7}{3}=\frac{1}{3}$. Thus $d_{D\left[N^{+}(v)\right]}(x) \geq 1$. Similarly, $d_{D\left[N^{+}(v)\right]}(y) \geq 1$. Therefore either $(x, y)$ or $(y, x)$ must be in $A(D)$, contrary to Claim 1 (iii), and so the case $m=0$ is not possible.

Therefore, we must have $m=1$. By Claim 1(iii), we may assume that $\{(x, y),(y, x)\} \cap$ $A(D)=\emptyset$. By $(3.8)$, we have $\left|N^{+}(v)\right|=3$, and so by $(3.5), d_{D\left[N^{+}(v)\right]}(x) \geq \frac{4(3)-7}{3}=\frac{5}{3}$. Thus $d_{D\left[N^{+}(v)\right]}(x) \geq 2$. Similarly, $d_{D\left[N^{+}(v)\right]}(y) \geq 2$. As $m=1$ and as $\{(x, y),(y, x)\} \cap A(D)=\emptyset$, either $X=\left\{x^{\prime}\right\}$ and $Y=\emptyset$, whence $\left\{\left(y, x^{\prime}\right),\left(x^{\prime}, y\right)\right\} \subset A(D)$, contrary to Claim 1(i); or $X=\emptyset$ and $Y=\left\{y^{\prime}\right\}$, whence $\left\{\left(x, y^{\prime}\right),\left(y^{\prime}, x\right)\right\} \subset A(D)$, contrary to Claim 1(ii). This completes the proof of Claim 3.

By Claim 3, we assume that $m \geq 2$. For some integers $s \geq 1$ and $t$ with $t \in\{-1,0,1\}$, we write $m=h+k=3 s+t$. By (3.8), $n_{v}=\left|N^{+}(v)\right|=m+2=3 s+t+2$. By (3.5), for each $z \in N^{+}(v), d_{D\left[N^{+}(v)\right]}(z) \geq \frac{4\left(n_{v}\right)-7}{3}=4 s+t+\frac{t+1}{3} \geq 4 s+t$. 
Case 1. $s>k$.

Then for some integer $k^{\prime} \geq 1, s=k+k^{\prime}$. as $h+k=m=3\left(k+k^{\prime}\right)+t$, we have $h=2 k+3 k^{\prime}+t$. By (3.5), $d_{D\left[N^{+}(v)\right]}(x) \geq 4 s+t=4 k+4 k^{\prime}+t=2 k+h+k^{\prime}$. Since $\left|(x, X)_{D} \cup(X, x)_{D}\right| \leq 2 k$ and as $k^{\prime}>0$, it follows that for some $y^{\prime} \in Y$, both $\left(x, y^{\prime}\right)$ and $\left(y^{\prime}, x\right)$ are in $A(D)$ or $\{(x, y),(y, x)\} \cap A(D) \neq \emptyset$, contrary to Claim 1(ii) or (iii). This proves that Case 1 cannot occur.

Case 2. $s=k$.

As $m=k+h$, this is equivalent to $h=2 k+t$, and so $2 k+h=2 h-t$. By (3.8), $n_{v}=m+2=3 k+t+2$. By $(3.5), d_{\left[N^{+}(v)\right]}(x) \geq \frac{4(3 k+t+2)-7}{3}=2 k+h+\frac{t+1}{3}$. Similarly, $d_{\left[N^{+}(v)\right]}(y) \geq 2 h-t+\frac{t+1}{3}=2 h+\frac{1-2 t}{3}$. For $t \in\{-1,0\}$, this is a violation to Claim 2. And for $t=1$, this is a violation to Claim 1 (ii) or 1 (iii). This proves that Case 2 cannot occur.

Case 3. $s<k$, and either $k^{\prime \prime} \geq 2$ or both $k^{\prime \prime}=1$ and $t \neq 1$ such that $k^{\prime \prime}=k-s$

Then $k>k^{\prime \prime} \geq 1, s=k-k^{\prime \prime}$. as $h+k=m=3\left(k-k^{\prime \prime}\right)+t$, we have $h=2 k-3 k^{\prime \prime}+t$. By $(3.5)$, $d_{D\left[N^{+}(v)\right]}(y) \geq 4 s+t=4 k-4 k^{\prime \prime}+t=\left(4 k-6 k^{\prime \prime}+2 t\right)+k^{\prime \prime}+\left(k^{\prime \prime}-t\right)=2 h+k^{\prime \prime}+\left(k^{\prime \prime}-t\right) \geq 2 h+k^{\prime \prime}+1 ;$ and $d_{D\left[N^{+}(v)\right]}(x) \geq 4 s+t=2 k+\left(2 k-3 k^{\prime \prime}+t\right)-k^{\prime \prime}=2 k+h-k^{\prime \prime}$. Since $\left|(y, Y)_{D} \cup(Y, y)_{D}\right| \leq 2 h$, and since $d_{D\left[N^{+}(v)\right]}(y) \geq 2 h+k^{\prime \prime}+1$, we have $\left|(y, X)_{D} \cup(X, y)_{D}\right| \geq k^{\prime \prime}+1$. By Claim 1(ii), $\left|(x, Y)_{D} \cup(Y, x)_{D}\right| \leq h$. As $d_{D\left[N^{+}(v)\right]}(x) \geq 2 k+h-k^{\prime \prime}$, we have $\left|(x, X)_{D} \cup(X, x)_{D}\right| \geq 2 k-k^{\prime \prime}$. This implies that there are at least $k-k^{\prime \prime}$ vertices $x^{\prime} \in X$ such that $\left(x, x^{\prime}\right),\left(x^{\prime}, x\right) \in A(D)$. As $\left|(y, X)_{D} \cup(X, y)_{D}\right| \geq k^{\prime \prime}+1$, there must be an $x^{\prime} \in X$ with $\left(x, x^{\prime}\right),\left(x^{\prime}, x\right) \in A(D)$ such that $\left|\left\{\left(y, x^{\prime}\right),\left(x^{\prime}, y\right)\right\} \cap A(D)\right| \geq 1$, contrary to Claim 1(i). Hence Case 3 does not hold.

Case 4. $s=k-1$ and $m=k+h=3 k-2$.

By (3.8), $h+k=m=3 s+t=3 k-2$, and so $h=2 k-2$. By (3.5), for each $z \in N^{+}(v)$ $d_{D\left[N^{+}(v)\right]}(z) \geq 4 s+t+\frac{t+1}{3}=4 k-\frac{7}{3}$. Since $d_{D\left[N^{+}(v)\right]}(z)$ is an integer, we have $d_{D\left[N^{+}(v)\right]}(z) \geq 4 k-$ 2. It follows that $d_{D\left[N^{+}(v)\right]}(x) \geq 2 k+(2 k-2)=2 k+h$ and $d_{D\left[N^{+}(v)\right]}(y) \geq 2(2 k-2)+2=2 h+2$, contrary to Claim 2. Thus Case 4 also leads to a contradiction.

As every case leads to a contradiction, this establishes the theorem.

\section{Proof of Corollary 3.1.2.}

Let $D$ be a locally $\left(\frac{2}{3}, 0\right)^{+}$-arc-connected strong simple digraph. Then by definition of locally $(\alpha, \beta)^{+}$-arc-connected strong simple digraph Corollary 3.1.2 means that $\forall v \in V(D)$, $\lambda\left(D\left[N^{+}(v)\right]\right) \geq \frac{2}{3}\left|N^{+}(v)\right|+0$ which by (3.1) implies that $\forall v \in V(D), d_{\left[N^{+}(v)\right]}(u) \geq 2\left(\frac{2}{3}\right)\left|N^{+}(v)\right|=$ 
$\frac{4}{3}\left|N^{+}(v)\right| \geq \frac{4}{3}\left|N^{+}(v)\right|-\frac{7}{3}, \forall u \in N^{+}(v)$. it follows that $D$ is a locally $\left(\frac{4}{3}, \frac{-7}{3}\right)^{+}$-dense strong simple digraph. By Theorem 3.1.1, D is supereulerian.

With similar arguments to those in the proof of Theorem 3.1.1, the following theorem can also be justified.

Theorem 3.3.1. Every locally $\left(\frac{4}{3}, \frac{-7}{3}\right)$-dense strong simple digraph is supereulerian.

Sketch of Proof. Assume that

$$
D \text { is a locally }\left(\frac{4}{3}, \frac{-7}{3}\right) \text {-dense strong simple digraph. }
$$

Since $D$ is strong, $D$ must have an eulerian subdigraph. Let $S$ be an eulerian subdigraph of $D$ such that among all eulerian subdigraphs of $D$

$$
|V(S)| \text { being maximized. }
$$

If $|V(S)|=|V(D)|$, then $S$ is a spanning eulerian subdigraph of $D$ and we are done. Assume by contradiction that $|V(D)|>|V(S)|>1$. Hence $V(D)-V(S) \neq \emptyset$. Since $D$ is strong and $S$ is a maximum eulerian subdigraph, there exists an $(S, S)$-path $Q$ on at least three vertices. Let $Q$ be chosen so that:

the length of a shortest dipath $P$ in $S$ between the endpoints of $Q$ is minimized.

Assume that $V(Q) \cap V(S)=\{v, z\}$ with $(v, x) \in A(Q)$. If $(v, z) \in A(S)$, then $S-(v, z)+A(Q)$ is an eulerian subdigraph with at least one more vertex than $S$, contrary to (3.10). Therefore, there must be a vertex $y \in V(P)-\{v, z\}$ such that $(v, y) \in A(P)$. Let $X=X^{+} \cup X^{-}$where $X^{+}=N^{+}(v)-(\{x, y\} \cup V(S))$ and $X^{-}=N^{-}(v)-V(S)$. Also let $Y=Y^{+} \cup Y^{-}$where $Y^{+}=N^{+}(v) \cap(V(S)-\{y\})$ and $Y^{-}=N^{-}(v) \cap V(S)$. As in the proof of Theorem 3.1.1, denote such that

$$
|X|=k,|Y|=h,|X|+|Y|=m=k+h,|N(v)|=n_{v}
$$

The rest of the proof will be identical to that of Theorem 3.1.1, using $N(v)$ instead of $N^{+}(v)$. Therefore, these similar arguments are omitted.

The final corollary follows from Theorem 3.3.1.

Corollary 3.3.2. Every locally $\left(\frac{2}{3}, 0\right)$-arc-connected strong simple digraph is supereulerian. 


\section{Chapter 4}

\section{Supereulerian Digraphs with Forbidden Induced Short Dipaths}

\subsection{Introduction}

We consider finite and simple digraphs. A digraph $D$ is simple if $D$ has no loops and if for any pair of distinct vertices $u, v \in V(D)$, there is at most one arc in $D$ oriented from $u$ to $v$. Undefined terms and notations will follow [4]. We use $(u, v)$ to represent an arc oriented from a vertex $u$ to a vertex $v$. For an integer $n>0$, we use $K_{n}^{*}$ to denote the complete digraph on $n$ vertices. Hence for every pair of distinct vertices $u, v \in V\left(K_{n}^{*}\right)$, there is exactly one arc $(u, v)$ in $A\left(K_{n}^{*}\right)$.

Following [4], for a digraph $D$ with $X, Y \subseteq V(D)$, define

$$
(X, Y)_{D}=\{(x, y) \in A(D): x \in X, y \in Y\}
$$

When $Y=V(D)-X$, we define

$$
\partial_{D}^{+}(X)=(X, V(D)-X)_{D} \text { and } \partial_{D}^{-}(X)=(V(D)-X, X)_{D}
$$

For a vertex $v \in V(D), d_{D}^{+}(v)=\left|\partial_{D}^{+}(\{v\})\right|$ and $d_{D}^{-}(v)=\left|\partial_{D}^{-}(\{v\})\right|$ are the out-degree and the indegree of $v$ in $D$, respectively. Finally, we define the following notations: $\delta^{+}(D)=\min \left\{d_{D}^{+}(v)\right.$ : $v \in V(D)\}$ and $\delta^{-}(D)=\min \left\{d_{D}^{-}(v): v \in V(D)\right\}$. Let $N_{D}^{+}(v)=\{u \in V(D)-v:(v, u) \in A(D)\}$ and $N_{D}^{-}(v)=\{u \in V(D)-v:(u, v) \in A(D)\}$ denote the out-neighbours and in-neighbours of $v$ in $D$, respectively. We call the vertices in $N_{D}^{+}(v), N_{D}^{-}(v)$ the out-neighbours, in-neighbours of $v$. When the digraph $D$ is understood from the context, we often omit the subscript $D$. 
The purpose of this chapter is to investigate forbidden induced subdigraph conditions to assure the existence of supereulerian digraphs. For an integer $k \geq 2$, let $P_{k}$ denote the dipath on $k$ vertices. A subdigraph $H$ of a digraph $D$ is a $P_{k}$-subdigraph if $H \cong P_{k}$. If $D$ does not have an induced $P_{k}$, then for any $P_{k}$-subdigraph $H$ of $D$, we must have $|A(D[V(H)])| \geq k$.

Definition 4.1.1. For integers $h \geq k \geq 2$, we define $\mathcal{F}\left(P_{k}, h\right)$ to be the family of all simple digraphs such that $D \in \mathcal{F}\left(P_{k}, h\right)$ if and only if $D$ is strong and satisfies both of the following.

(i) $D$ contains at least one dipath $P_{k}$ with $\left|A\left(D\left[V\left(P_{k}\right)\right]\right)\right|=h$, and

(ii) for any dipath $P_{k}$ in $D,\left|A\left(D\left[V\left(P_{k}\right)\right]\right)\right| \geq h$.

By definition, it follows that $k \leq h \leq k(k-1)$; and by our proofs we will find that if there exists an $h_{k}$ such that every digraph in $\mathcal{F}\left(P_{k}, h_{k}\right)$ is supererlerian, then every digraph in $\mathcal{F}\left(P_{k}, h\right)$ is supererlerian when $h_{k} \leq h \leq k(k-1)$. In sections 2 and 3 , we will determine for each integer $k \in\{2,3,4\}$, the smallest integer $h_{k}$ such that every digraph in $\mathcal{F}\left(P_{k}, h_{k}\right)$ is supereulerian.

\subsection{Supereulerian Digraph in $\mathcal{F}\left(P_{2}, h\right)$ and $\mathcal{F}\left(P_{3}, h\right)$}

In this section, we investigate the supereulerianicity of digraphs in $\mathcal{F}\left(P_{2}, h\right)$ with $h=2$ and in $\mathcal{F}\left(P_{3}, h\right)$ with $3 \leq h \leq 6$. We need a necessary condition for a digraph to be supereulerian. Let $D$ be a digraph and $U \subset V(D)$. We call a collection of ditrails $T_{1}, T_{2}, \cdots, T_{t}$ of the induced subdigraph $D[U]$ a cover of $U$ if $\cup_{i=1}^{t} V\left(T_{i}\right)=U$ and $A\left(T_{i}\right) \cap A\left(T_{j}\right)=\emptyset$, whenever $i \neq j$. The minimum value of such $t$ is denoted by $\tau(U)$. For any subset $A \subseteq V(D)-U$, define $B=: V(D)-U-A$. Let

$$
h(U, A)=: \min \left\{\left|\partial_{D}^{+}(A)\right|,\left|\partial_{D}^{-}(A)\right|\right\}+\min \left\{\left|(U, B)_{D}\right|,\left|(B, U)_{D}\right|\right\}-\tau(U) .
$$

Then we have the following proposition.

Proposition 4.2.1. (Hong, Lai and Liu, Proposition 2.1 of [16]) If D has a spanning eulerian subdigraph, then for any $U \subset V(D)$, we have $h(U, A) \geq 0$.

Digraphs in $\mathcal{F}\left(P_{3}, h\right)$ with $3 \leq h \leq 4$ are not necessarily supereulerian, as can be seen in the example below. 


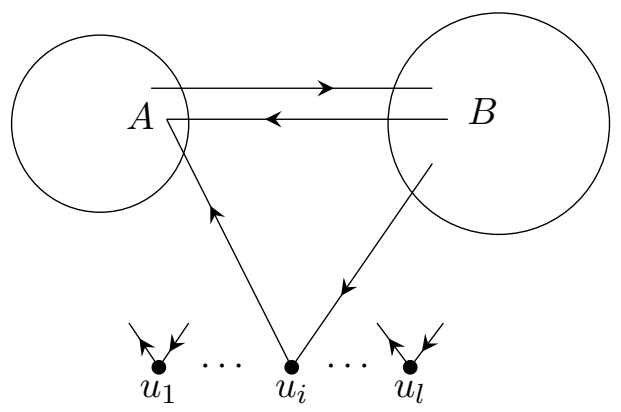

Figure 4.1. The digraph $D=D(\alpha, \beta, k, \ell)$.

Example 4.2.2. Let $\alpha, \beta, k>0$ be integers with $\alpha, \beta \geq k+1$, and let $A$ and $B$ be two disjoint set of vertices with $|A|=\alpha$ and $|B|=\beta$. Let $\ell \geq \alpha \beta+1$ be an integer, and $U$ be a set of vertices disjoint from $A \cup B$ with $|U|=\ell$. We construct a digraph $D=D(\alpha, \beta, k, \ell)$ such that $V(D)=A \cup B \cup U$ and the arcs of $D$ are given as required in (D1) and (D2) below. (See Figure 4.1).

(D1) $D[A \cup B] \cong K_{\alpha+\beta}^{*}$ is a complete digraph.

(D2) For every vertex $u \in U$, and for every $v \in A,(u, v) \in A(D)$ and for every $w \in B$, $(w, u) \in A(D)$. Thus for any $u \in U$, we have $N_{D}^{+}(u)=A$ and $N_{D}^{-}(u)=B$. No two vertices in $U$ are adjacent.

Direct computation yields

$$
h(U, A)=\left|\partial_{D}^{+}(A)\right|+\left|(U, B)_{D}\right|-\tau(U)=\alpha \beta-|U|<0,
$$

and so by Proposition 4.2.1, any $D=D(\alpha, \beta, k, \ell)$ is nonsupereulerian. By definition4.1.1, $D \in \mathcal{F}\left(P_{3}, 4\right)$.

An $\operatorname{arc}(u, v)$ of $D$ is symmetric in $D$ if both $(u, v),(v, u) \in A(D)$. A digraph $D$ is symmetric if $|V(D)|=1$ or if $|A(D)|>0$ and every arc of $D$ is symmetric. A digraph $D \neq K_{1}$ is symmetrically connected, if for every $u, v \in V(D), D$ contains a symmetric dipath from $u$ to $v$.

Theorem 4.2.3. ([2]) If D is symmetrically connected, then $D$ is supereulerian.

We observe that if $D \in \mathcal{F}\left(P_{2}, 2\right) \cup \mathcal{F}\left(P_{3}, 5\right)$, then $D$ is symmetrically connected, and so by Theorem 4.2.3, every digraph in $\mathcal{F}\left(P_{2}, 2\right) \cup \mathcal{F}\left(P_{3}, 5\right)$ is supereulerian. Hence we have our conclusions in this section.

Proposition 4.2.4. Let $D$ be a digraph.

(i) Every digraph in $\mathcal{F}\left(P_{2}, 2\right) \cup \mathcal{F}\left(P_{3}, 5\right)$ is supereulerian.

(ii) Not every digraph in $\mathcal{F}\left(P_{3}, 3\right) \cup \mathcal{F}\left(P_{3}, 4\right)$ is supereulerian. 


\subsection{Supereulerian Digraph in $\mathcal{F}\left(P_{4}, h\right)$}

Throughout this section, $k>0$ denotes an integer. In this section, we will study the supereulerianicity of digraphs in $\mathcal{F}\left(P_{4}, h\right)$, and determine the smallest value of $h_{4}$ such that every digraph in $\mathcal{F}\left(P_{4}, h_{4}\right)$ is supereulerian. We start with some terminology and definitions. For a digraph $D$ and a subdigraph $S$ of $D$, an $(x, y)$-dipath $P$ is called an $(S, S)$-dipath if $V(P) \cap V(S)=\{x, y\}$. An $(S, S)$-dipath $P$ is shortest if for some $x, y \in V(S), P$ is an $(x, y)$-dipath and $P$ is shortest among all $(S, S)$-dipaths starting at $x$ and ending at $y$.

Definition 4.3.1. Let $D$ be a digraph. Suppose that $S$ is an eulerian subdigraph of $D$ with $|V(S)|$ maximized. A shortest $(S, S)$-dipath $H$ with $|V(H)|=k+2 \geq 3$ is called a $k$-handle of $S$ in $D$.

Definition 4.3.2. Let $D$ be a digraph with a $k$-handle $H$ such that $H$ is a shortest handle in D. Then we call $D$ a k-handle digraph.

The following is a necessary condition for a digraph to be supereulerian.

Lemma 4.3.3. A digraph $D$ is nonsupereulerian if there exist vertex-disjoint subdigraphs $\left\{A, B_{1}, \ldots, B_{m}\right\}$ of $D$, for some integer $m>0$, satisfying each of the following.

(i) $N^{-}\left(B_{i}\right) \subseteq V(A), \forall i \in\{1,2, \ldots, m\}$.

(ii) $\left|\partial^{-}(A)\right| \leq m-1$.

Proof. By contradiction, we assume that both (i) and (ii) hold and $D$ is supereulerian. Let $S$ be a spanning closed ditrail in $D$. By (ii), $\left|\partial^{-}(A) \cap A(S)\right| \leq m-1$. It follows from the fact that $S$ is eulerian that

$$
\left|\partial^{+}(A) \cap A(S)\right| \leq m-1 \text {. }
$$

By (i) and by (4.1), there exists a $B_{j}$ with $j \in\{1,2, \ldots, m\}$ such that $\partial^{-}\left(B_{j}\right) \cap A(S)=\emptyset$, contrary to the assumption that $V(S)=V(D)$.

In the rest of this section, we examine the supereulerian membership of digraphs in $\mathcal{F}\left(P_{4}, h\right)$, for each value $h$ with $4 \leq h \leq 12=\left|A\left(K_{4}^{*}\right)\right|$. Our goal is to find the smallest value $h_{4}$ such that every digraph in $\mathcal{F}\left(P_{4}, h_{4}\right)$ is supereulerian, and to show that there exist at least one nonsupereulerian digraph in $\mathcal{F}\left(P_{4}, h_{4}-1\right)$.

\section{Proposition 4.3.4.}


I) $\forall k \geq 1$ there exist infinite family of strong $k$-handle digraphs in $\mathcal{F}\left(P_{4}, 5\right)$.

II) There exist infinite family of strong 1-handle digraphs in $\mathcal{F}\left(P_{4}, 6\right)$.

III) There exist infinite family of nonsupereulerian digraphs in $\mathcal{F}\left(P_{4}, 7\right)$. More precisely, there are infinitely many 1-handles in $\mathcal{F}\left(P_{4}, 7\right)$.

Proof. I) (A) For $k=1$, let $M=x z y$ be a symmetric dipath, $Q=x u y$ be a dipath and $H_{i}=x v_{i} y, i \geq 1$ be dipaths. Let $D_{1}=M \cup Q \cup H_{1} \cup\{(u, z)\}$. For any $P_{4}$ in $D_{1},\left|A\left(D\left[V\left(P_{4}\right)\right]\right)\right| \geq 5$ and $\left|A\left(D\left[V\left(u z x v_{1}\right)\right]\right)\right|=5$ and by Lemma (4.3.3) let $A=x, B_{1}=u$ and $B_{2}=v_{1}$. Thus, $D_{1}$ is not supereulerian but it is 1 -handle. Let $D_{l}=D_{1} \cup\left\{H_{2}, \ldots, H_{l}\right\}$. Then $D_{l} \in \mathcal{F}\left(P_{4}, 5\right)$ and by Lemma (4.3.3) $D_{l}$ is not supereulerian but it is 1-handle as illustrated in Figure (4.2).

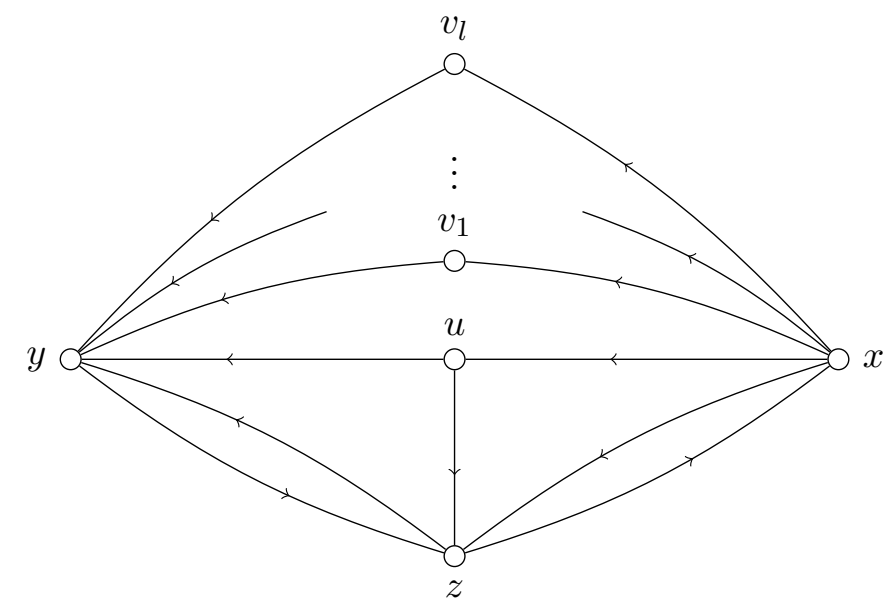

Figure 4.2. The digraph $D_{l}$.

I) (B) For $k=2$, let $M=x z y$ be a symmetric dipath, $Q=u_{1} u_{2}$ be symmetric dipath (arc) and $H_{i}=v_{i 1} v_{i 2}, i \geq 1$ be a symmetric dipaths (arcs). Let $D_{1}=M \cup Q \cup H_{1} \cup$ $\left\{\left(x, u_{1}\right),\left(x, v_{11}\right),(x, y),\left(u_{2}, y\right),\left(v_{12}, y\right)\right\}$. For any $P_{4}$ in $D_{1},\left|A\left(D\left[V\left(P_{4}\right)\right]\right)\right| \geq 5$ and $\left|A\left(D\left[V\left(x u_{1} u_{2} y\right)\right]\right)\right|=$ 5 and by Lemma (4.3.3) let $A=x, B_{1}=Q$ and $B_{2}=H_{1}$. Thus, $D_{1}$ is not supereulerian but it is 2-handle. Let $D_{l}=D_{1} \cup\left\{H_{2}, \ldots, H_{l}\right\} \cup\left(\bigcup_{j=2}^{l}\left\{\left(x, v_{j 1}\right),\left(v_{j 2}, y\right)\right\}\right)$. Then $D_{l} \in \mathcal{F}\left(P_{4}, 5\right)$ and by Lemma (4.3.3) $D_{l}$ is not supereulerian but it is 2-handle as illustrated in Figure (4.3). 


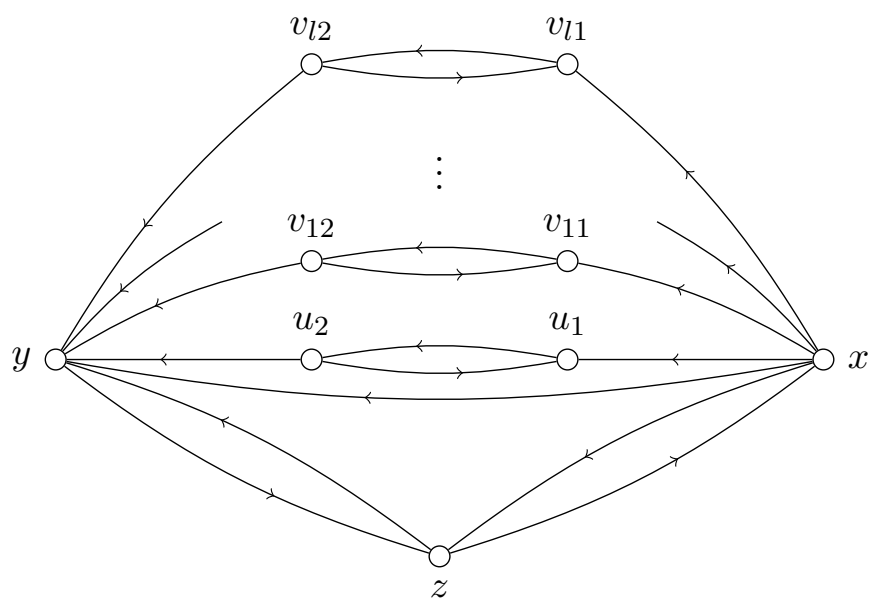

Figure 4.3. The digraph $D_{l}$.

I) (C) For $k \geq 3$, let $M=x z y$ be a symmetric dipath, $Q=u_{1}, \ldots, u_{k}$ be a symmetric dipath and $H_{i k}=v_{i 1}, \ldots, v_{i k}, i \geq 1$ be symmetric dipaths. Let $D_{1 k}=M \cup Q \cup H_{1 k} \cup$ $\left\{\left(x, u_{1}\right),\left(x, v_{11}\right),\left(u_{k}, y\right),\left(v_{1 k}, y\right)\right\}$. For any $P_{4}$ in $D_{1 k},\left|A\left(D_{1 k}\left[V\left(P_{4}\right)\right]\right)\right| \geq 5$ and $\left|A\left(D_{1 k}\left[V\left(y z x u_{1}\right)\right]\right)\right|=$ 5 and by Lemma (4.3.3) let $A=x, B_{1}=Q$ and $B_{2}=H_{1 k}$. Thus, $D_{1 k}$ is not supereulerian for every $k \geq 3$ but it is $k$-handle. Let $D_{l k}=D_{1 k} \cup\left\{H_{2 k}, \ldots, H_{l k}\right\} \cup\left(\cup_{j=2}^{l}\left\{\left(x, v_{j 1}\right),\left(v_{j k}, y\right)\right\}\right), l \geq 2$. Then $D_{l k} \in \mathcal{F}\left(P_{4}, 5\right)$ and by Lemma (4.3.3) $D_{l k}$ is not supereulerian but it is $k$-handle as illustrated in Figure(4.4).

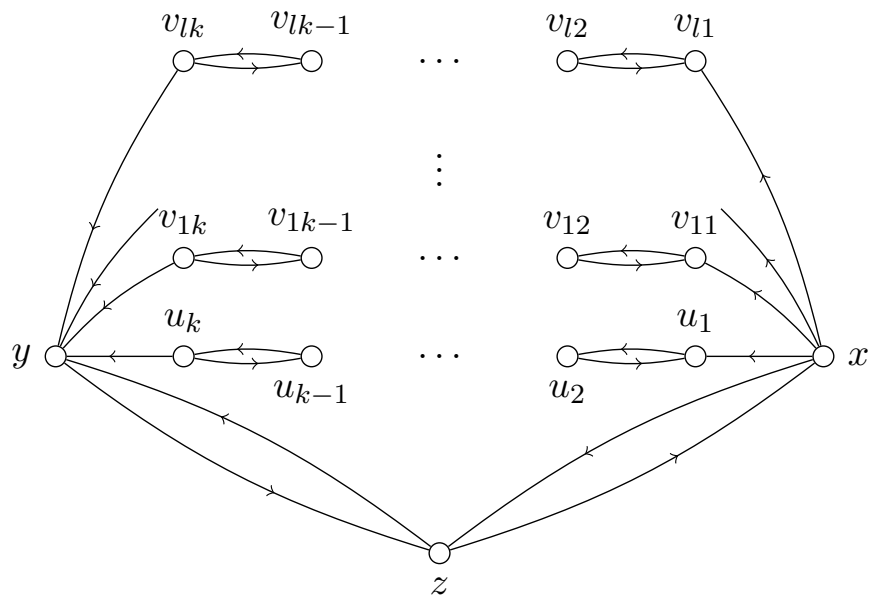

Figure 4.4. The digraph $D_{l k}$.

II) Let $M=x z y$ be a symmetric dipath, $Q=x u y$ be a dipath and $H_{i}=x v_{i} y, i \geq 1$ be dipaths. Let $D_{1}=M \cup Q \cup H_{1}$. For any $P_{4}$ in $D_{1},\left|A\left(D\left[V\left(P_{4}\right)\right]\right)\right|=6$ and by Lemma 
(4.3.3) let $A=x, B_{1}=u$ and $B_{2}=v_{1}$. Thus, $D_{1}$ is not supereulerian but it is 1 -handle. Let $D_{l}=D_{1} \cup\left\{H_{2}, \ldots, H_{l}\right\}$. Then $D_{l} \in \mathcal{F}\left(P_{4}, 6\right)$ and by Lemma (4.3.3) $D_{l}$ is not supereulerian but it is 1-handle as illustrated in Figure (4.5).

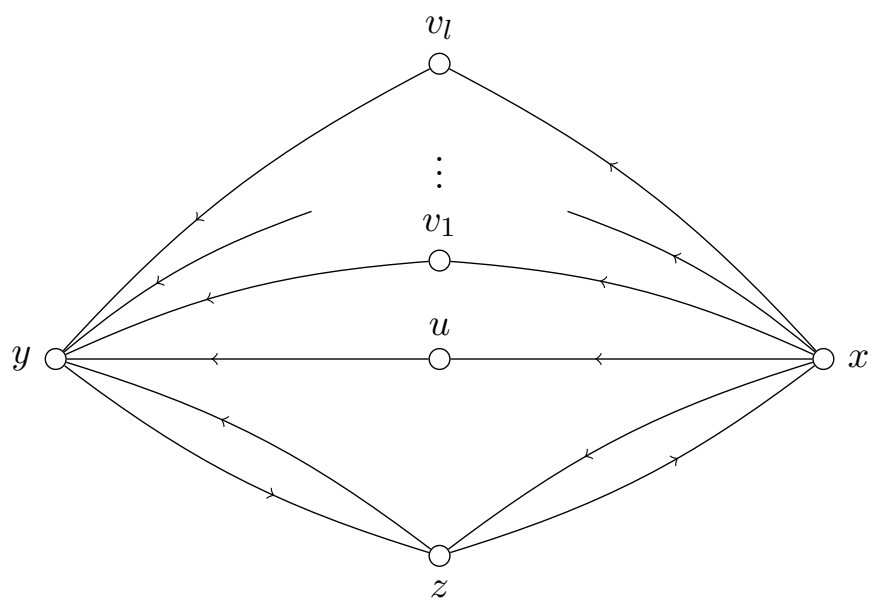

Figure 4.5. The digraph $D_{l}$.

III) Let $M=x z y$ be a symmetric dipath, $Q=x u y$ be a dipath and $H_{i}=x v_{i} y, 1 \leq i \leq \ell$ be dipaths. Let $D_{\ell}=M \cup Q \cup\left(\bigcup_{i=1}^{\ell} H_{i}\right) \cup\{(x, y)\}$. See in Figure 4.6. It is routine to verify that $D_{\ell} \in \mathcal{F}\left(P_{4}, 7\right)$. By Lemma (4.3.3) with $A=D[\{x\}], B_{1}=D[\{u\}]$ and $B_{2}=D\left[\left\{v_{1}\right\}\right]$, we conclude that $D_{\ell}$ is nonsupereulerian. By Definition 4.3.1, $D_{\ell}$ is 1-handle.

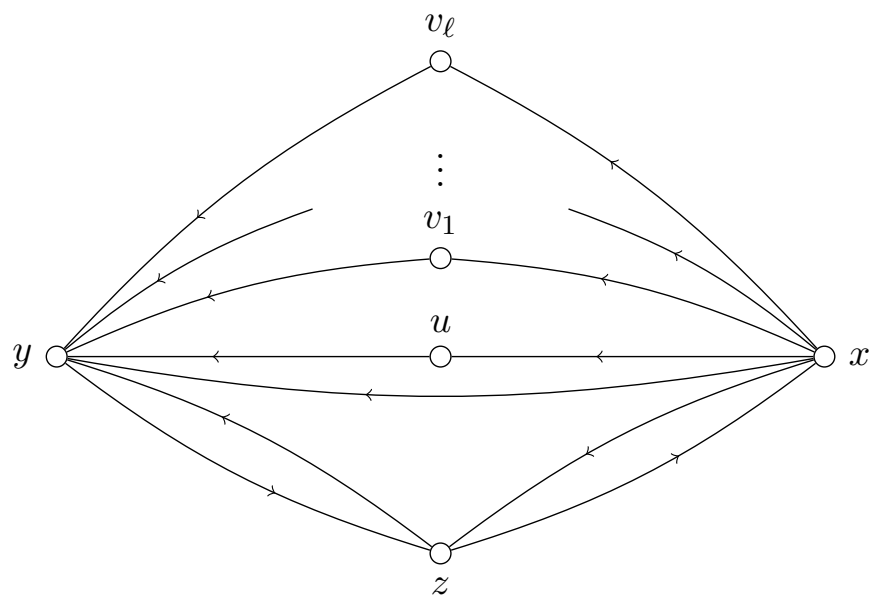

Figure 4.6. The digraph $D_{\ell}$. 
Observation 4.3.5. Suppose that $D$ is a nonsupereulerian strong digraph. Let $S$ be a maximum eulerian subdigraph of $D$ and let $H=x u_{1} \ldots u_{k} y$ be $a k$-handle of $S$ and $Q=x v_{1} \ldots v_{s} y$ be a shortest (x,y)-dipath in $S$ with $k+s$ is minimized. We have the following observations.

(A) If for some $i$ with $1 \leq i \leq k$, we have $\left\{\left(u_{i}, x\right),\left(y, u_{i}\right)\right\} \cap A(D) \neq \emptyset$, then $S \cup\left\{\left(x, u_{1}\right),\left(u_{1}, u_{2}\right), \ldots\right.$, $\left.\left(u_{i-1}, u_{i}\right),\left(u_{i}, x\right)\right\}$ or $S \cup\left\{\left(y, u_{i}\right),\left(u_{i}, u_{i+1}\right), \ldots,\left(u_{k-1}, u_{k}\right),\left(u_{k}, y\right)\right\}$ would violate the maximality of $S$. Hence for any $i$ with $1 \leq i \leq k$, we have $\left\{\left(u_{i}, x\right),\left(y, u_{i}\right)\right\} \cap A(D)=\emptyset$.

(B) If for some $i$ with $1 \leq i \leq k-1$, we have $\left\{\left(x, u_{i+1}\right),\left(u_{i}, y\right)\right\} \cap A(D) \neq \emptyset$, then $H^{\prime}=$ $x u_{i+1} \ldots u_{k} y$ or $H^{\prime \prime}=x u_{1} \ldots u_{i} y$ is a shorter $(S, S)$-dipath, contrary to the fact that $H$ is a shortest $(S, S)$-dipath, stated in Definition 4.3.1. Hence for any $1 \leq i \leq k-1$, we have $\left\{\left(x, u_{i+1}\right),\left(u_{i}, y\right)\right\} \cap A(D)=\emptyset$.

(C) By Definition 4.3.1, $H$ is a shortest $(S, S)$-dipath. The minimality of $k+s$ implies that for every $i, j$ with $1 \leq i \leq s, 1 \leq j \leq k$, we have $\left\{\left(v_{i}, u_{j}\right),\left(u_{j}, v_{i}\right)\right\} \cap A(D)=\emptyset$; and for every $j$ with $i+2 \leq j$, we have $\left(u_{i}, u_{j}\right) \notin A(D)$.

Theorem 4.3.6. Every digraph $D$ in $\mathcal{F}\left(P_{4}, 8\right)$ is supereulerian.

Proof. Assume that $D \in \mathcal{F}\left(P_{4}, 8\right)$. By contradiction, assume that $D$ is a nonsupereulerian digraph. Let $S$ be a maximum eulerian subdigraph of $D$ and let $H=x u_{1} \ldots u_{k} y$ be a $k$-handle of $S$ and

$$
Q=x v_{1} \ldots v_{s} y \text { be a shortest }(x, y) \text { - dipath in } S \text { with } k+s \text { is minimized }(s \geq 1) .
$$

We consider three cases.

Case 1. $k \geq 3$.

In this case, $P^{\prime}=x u_{1} u_{2} u_{3}$ is a $P_{4}$ in $D$. By Observation 4.3.5 $((A)$ and $(B))$, we conclude that $\left\{\left(u_{1}, x\right),\left(x, u_{2}\right),\left(u_{2}, x\right),\left(x, u_{3}\right),\left(u_{3}, x\right)\right\} \cap A(D)=\emptyset$. It follows that $\left|A\left(D\left[V\left(P^{\prime}\right)\right]\right)\right|<8$, contrary to the assumption that $D \in \mathcal{F}\left(P_{4}, 8\right)$.

Case 2. $k=2$.

In this case, $P^{\prime \prime}=x u_{1} u_{2} y$ is a $P_{4}$ in $D$. By Observation 4.3.5 $((A)$ and $(B))$, we conclude that $\left\{\left(u_{1}, x\right),\left(x, u_{2}\right),\left(u_{2}, x\right),\left(y, u_{2}\right),\left(y, u_{1}\right),\left(u_{1}, y\right)\right\} \cap A(D)=\emptyset$. It follows that $\left|A\left(D\left[V\left(P^{\prime \prime}\right)\right]\right)\right|<8$, contrary to the assumption that $D \in \mathcal{F}\left(P_{4}, 8\right)$.

Case 3. $k=1$.

Claim 1. $(y, x) \notin A(D)$.

By contradiction, we assume that $(y, x) \in A(D)$. If $(y, x) \notin A(S)$, then $S \cup A(H) \cup\{(y, x)\}$ 
is violation to the maximality of $S$. Hence $(y, x) \in A(S)$. Since $P^{(3)}=u_{1} y x v_{1}$ is a $P_{4}$ in $D$, by Observation 4.3.5 $((A)$ and $(C))$, we conclude that $\left\{\left(u_{1}, x\right),\left(y, u_{1}\right),\left(u_{1}, v_{1}\right),\left(v_{1}, u_{1}\right)\right\} \cap A(D)=\emptyset$. Since $D \in \mathcal{F}\left(P_{4}, 8\right)$, we have $\left.\mid A\left(D\left[V\left(P^{(3)}\right)\right)\right]\right) \mid \geq 8$, and so $\left\{\left(y, v_{1}\right),\left(v_{1}, x\right)\right\} \subset A(D)$.

Suppose first that $s=1$. If $\left\{\left(y, v_{1}\right),\left(v_{1}, x\right)\right\} \cap A(S)=\emptyset$, then $S \cup\left\{\left(y, v_{1}\right),\left(v_{1}, x\right),\left(x, u_{1}\right),\left(u_{1}, y\right)\right\}$ is a violation to the maximality of $S$. Hence $\left\{\left(y, v_{1}\right),\left(v_{1}, x\right)\right\} \cap A(S) \neq \emptyset$, whence $S-\left\{\left(x, v_{1}\right),\left(v_{1}, y\right)\right\}+$ $\left\{\left(x, u_{1}\right),\left(u_{1}, y\right)\right\}$ is a violation to the maximality of $S$. In either case, a contradiction obtains and so we must have $s \geq 2$. Note that $P^{(4)}=u_{1} y v_{1} v_{2}$ is a $P_{4}$ in $D$. By Observation 4.3.5 $((A)$ and $(C))$, we conclude that $\left\{\left(y, u_{1}\right),\left(u_{1}, v_{1}\right),\left(v_{1}, u_{1}\right),\left(u_{1}, v_{2}\right),\left(v_{2}, u_{1}\right)\right\} \cap A(D)=\emptyset$. It follows that $\left|A\left(D\left[V\left(P^{(4)}\right)\right]\right)\right|<8$, contrary to the assumption that $D \in \mathcal{F}\left(P_{4}, 8\right)$. This justifies Claim 1.

Claim 2. For any $i$ with $1 \leq i \leq s,\left(y, v_{i}\right) \notin A(D)$.

By contradiction, we assume that $\left(y, v_{i}\right) \in A(D)$ for some $i$ with $1 \leq i \leq s$. Then $P^{(5)}=x u_{1} y v_{i}$ is a $P_{4}$ in $D$. Since $D \in \mathcal{F}\left(P_{4}, 8\right)$, we must have $\left|A\left(D\left[V\left(P^{(5)}\right)\right]\right)\right| \geq 8$. By Observation 4.3.5 $((A)$ and $(C))$ and Claim $1,\left\{\left(u_{1}, x\right),\left(y, u_{1}\right),\left(u_{1}, v_{i}\right),\left(v_{i}, u_{1}\right),(y, x)\right\} \cap A(D)=\emptyset$. It follows that $\left|A\left(D\left[V\left(P^{(5)}\right)\right]\right)\right|<8$, contrary to the assumption that $D \in \mathcal{F}\left(P_{4}, 8\right)$. This justifies Claim 2.

By Claims 1 and 2, we have,

$$
\left\{\left(y, v_{1}\right),\left(y, v_{2}\right), \cdots,\left(y, v_{s}\right),(y, x)\right\} \cap A(D)=\emptyset .
$$

Claim 3. For any $z \in V(D),(y, z) \notin A(D)$.

By contradiction, we assume that for some $z \in V(D)$, we have $(y, z) \in A(D)$. Then $P^{(6)}=x u_{1} y z$ is a $P_{4}$ in $D$. Since $D \in \mathcal{F}\left(P_{4}, 8\right)$, we have $\left|A\left(D\left[V\left(P^{(6)}\right)\right]\right)\right| \geq 8$.

By Observation 4.3.5 $(A)$ and by (4.3), we have $\left\{\left(u_{1}, x\right),\left(y, u_{1}\right),(y, x)\right\} \cap A(D)=\emptyset$. It follows that $\left|\left\{\left(z, u_{1}\right),\left(u_{1}, z\right)\right\} \cap A(D)\right| \geq 1$.

Suppose first that $\left\{\left(z, u_{1}\right),\left(u_{1}, z\right)\right\} \subseteq A(D)$. If $z \notin V(S)$, then $S \cup\left\{(y, z),\left(z, u_{1}\right),\left(u_{1}, y\right)\right\}$ violates the maximality of $S$. If $z \in V(S)$, then $S \cup\left\{\left(z, u_{1}\right),\left(u_{1}, z\right)\right\}$ violates the maximality of $S$. We have $\left|\left\{\left(z, u_{1}\right),\left(u_{1}, z\right)\right\} \cap A(D)\right|=1$. Since $D \in \mathcal{F}\left(P_{4}, 8\right)$, therefore, we have $\left|A\left(D\left[V\left(P^{(6)}\right)\right]\right)\right| \geq 8$. Thus $\left\{(z, y),(z, x),(x, z),(x, y),\left(x, u_{1}\right),\left(u_{1}, y\right),(y, z)\right\} \subseteq A(D)$. Since $\left|\left\{\left(z, u_{1}\right),\left(u_{1}, z\right)\right\} \cap A(D)\right|=1$, we consider just two subcases.

Subcase 3.1. $\left(z, u_{1}\right) \in A(D)$ but $\left(u_{1}, z\right) \notin A(D)$. 
If $z \notin V(S)$, then $S \cup\left\{(y, z),\left(z, u_{1}\right),\left(u_{1}, y\right)\right\}$ violates the maximality of $S$. Hence we assume that $z \in V(S)$. The dipath $P^{(7)}=v_{s} y z u_{1}$ is a $P_{4}$ in $D$. By Observation 4.3.5 $((A)$ and $(C))$ and by (4.3), we have $\left\{\left(u_{1}, z\right),\left(v_{s}, u_{1}\right),\left(u_{1}, v_{s}\right),\left(y, u_{1}\right),\left(y, v_{s}\right)\right\} \cap A(D)=\emptyset$. It follows that $\left|A\left(D\left[V\left(P^{(7)}\right)\right]\right)\right|<8$, contrary to the assumption that $D \in \mathcal{F}\left(P_{4}, 8\right)$. This contradiction indicates that Subcase 3.1 does not occur.

Subcase 3.2. $\left(u_{1}, z\right) \in A(D)$ but $\left(z, u_{1}\right) \notin A(D)$.

Note that $P^{(8)}=u_{1} z x v_{1}$. Since $D \in \mathcal{F}\left(P_{4}, 8\right)$, we have $\left|A\left(D\left[V\left(P^{(8)}\right)\right]\right)\right| \geq 8$. By Observation 4.3.5 $((A)$ and $(C))$ and by $(4.3),\left\{\left(u_{1}, x\right),\left(u_{1}, v_{1}\right),\left(v_{1}, u_{1}\right),\left(z, u_{1}\right)\right\} \cap A(D)=\emptyset$, and so $\left(z, v_{1}\right) \in$ $A(D)$.

Recall that $Q$ is a shortest $(x, y)$-dipath in $S$ as defined in (4.2). The dipath $P^{(9)}=u_{1} z v_{1} v_{2}$ is a $P_{4}$ in $D$, where $v_{2}=y$ when $Q=x v_{1} y$. Since $D \in \mathcal{F}\left(P_{4}, 8\right)$, we have $\left|A\left(D\left[V\left(P^{(9)}\right)\right]\right)\right| \geq$ 8. By Observation 4.3.5 $(C)$ and by (4.3), $\left\{\left(u_{1}, v_{1}\right),\left(v_{1}, u_{1}\right),\left(v_{2}, u_{1}\right),\left(z, u_{1}\right)\right\} \cap A(D)=\emptyset$, where $\left(u_{1}, v_{2}\right) \notin A(D)$ when $v_{2} \neq y$ and $\left(y, v_{1}\right) \notin A(D)$ when $v_{2}=y$. These imply that $\left|A\left(D\left[V\left(P^{(9)}\right)\right]\right)\right|<8$, contrary to the assumption that $D \in \mathcal{F}\left(P_{4}, 8\right)$. 


\section{Chapter 5}

\section{Degree Condition for Supereulerian Digraph}

\subsection{Introduction}

We consider finite and simple digraphs (without loops or parallel arcs, but possibly with cycles of length two). Given a digraph $D$ we denote by $V(D), A(D)$ its vertex set and arc set, respectively, when $D$ is clear from context we simply write $V$ and $A$. Undefined terms and notations will follow [4]. Throughout this chapter, we use the notation $(u, v)$ to denote an arc oriented from $u$ to $v$ in a digraph. When $(u, v) \in A(D)$, we say that $u$ and $v$ are adjacent.

For digraphs $H$ and $D$, by $H \subseteq D$ we mean that $H$ is a subdigraph of $D$. Following [4], for a digraph $D$ with $X, Y \subseteq V(D)$, define

$$
(X, Y)_{D}=\{(x, y) \in A(D): x \in X, y \in Y\}
$$

When $Y=V(D)-X$, we define

$$
\partial_{D}^{+}(X)=(X, V(D)-X)_{D} \text { and } \partial_{D}^{-}(X)=(V(D)-X, X)_{D}
$$

For a vertex $v \in V(D), d_{D}^{+}(v)=\left|\partial_{D}^{+}(\{v\})\right|$ and $d_{D}^{-}(v)=\left|\partial_{D}^{-}(\{v\})\right|$ are the out-degree and the in-degree of $v$ in $D$, respectively.

A digraph $D$ is complete if, for every pair $x, y$ of distinct vertices of $D$ both $(x, y)$ and $(y, x)$ are in $D$. The complete digraph on $n$ vertices will be denoted by $K_{n}^{*}$. Given an $(x, y)$-dipath $P$, we denote by $P] x, y\left[\right.$ the dipath $P-\{x, y\}$. Given a dipath $P=v_{1}, v_{2}, \ldots, v_{k}$ we denote by 
$P\left[v_{i}, v_{j}\right]$, where $1 \leq i<j \leq k$ the subdipath of $P$ starts at $v_{i}$ and ends at $v_{j}$. For a subdigraph $H$ of a digraph $D$, an $(x, y)$-dipath $P$ is an $(H, H)$-dipath if $x, y \in V(H)$ and $V(P) \cap V(H)=\{x, y\}$. We say that an ordered pair of vertices $(x, y)$ is dominated (dominating) if there exists $z \in V(D)$, with $(z, x),(z, y) \in A(D)((x, z),(y, z) \in A(D))$.

A walk in $D$ is an alternating sequence $W=x_{1} a_{1} x_{2} a_{2} \ldots x_{k-1} a_{k-1} x_{k}$ of vertices $x_{i}$ and arcs $a_{j}$ from $D$ such that $a_{i}=x_{i} x_{i+1}$ for $i=1, \ldots, k-1$. A walk is closed if $x_{1}=x_{k}$, and open otherwise. If all the arcs of a walk are distinct we call it a ditrail. If a ditrail starts at $s$ and ends at $t$ we call it $(s, t)$-ditrail. Given a ditrail $T$ and two vertices $a \neq b$ such that $T$ contains some $(a, b)$-ditrails, we denote by $T[a, b]$ these ditrails.

Let $H$ be a subdigraph of a digraph $D$ and $X \subseteq A(D)$. Then we use $H+X$ to denote the subdigraph $D[A(H) \cup X]$ induced by $A(H) \cup X$. If $H^{\prime}$ is also a subdigraph of $D$, then we use $H+H^{\prime}$ for $H+A\left(H^{\prime}\right)$.

The property of being supereulerian is at the same time relaxation of being hamiltonian: being supereulerian digraph means having a closed ditrail covering all the vertices of the digraph; being hamiltonian means having a closed ditrail covering all vertices of the digraph without using a vertex twice. In this chapter we analyze some sufficient conditions for a digraph to be supereulerian. Due to the analogy with hamiltonian cycles, our main result and its proof technique, are similar to some results, and proofs, in hamiltonian and supereulerian digraph theory.

The purpose of the following section is to show that, as it is the case for undirected graphs, some sufficient degree conditions for hamiltonicity in digraphs can be (slightly) weakened to become sharp sufficient conditions for supereulerianity. The next well known theorem in hamiltonian digraphs is due to Meyniel.

Theorem 5.1.1. (Meyniel [19]) A strong digraph $D$ on $n$ vertices satisfying $d(x)+d(y) \geq 2 n-1$ for all pairs of non-adjacent vertices $x, y$ is hamiltonian.

Bang-Jensen and Maddaloni in [5] proved the following theorem which is a similar result to Meyniel's theorem for supereulerian digraphs.

Theorem 5.1.2. (J. Bang-Jensen and A. Maddaloni [5]) Let D be a strong simple digraph on $n$ vertices. If $d(x)+d(y) \geq 2 n-3$ for any pair of non adjacent vertices $x$ and $y$, then $D$ is supereulerian.

The next theorem is due to Zhao and Meng. 
Theorem 5.1.3. (Zhao and Meng [24]) Let D be a strong digraph of order $n \geq 2$. If

$$
d^{+}(x)+d^{+}(y)+d^{-}(u)+d^{-}(v) \geq 2 n-1
$$

for every pair $x, y$ of dominating vertices and every pair $u, v$ of dominated vertices, then $D$ is hamiltonian.

We study when this kind of conditions in theorem 5.1.3 is sufficient for a digraph to be supereulerian. Our proof of the main theorem use an approach similar to the one used in [5] and the following lemma which is a generalization of a lemma in [5] dealt with a vertex and ditrail.

Lemma 5.1.4. Let $D$ be a digraph. Let $s, t, x, y \in V(D)$ and let $T$ be an $(s, t)-$ ditrail in $D$ and let $P$ be an $(x, y)$-dipath in $D$ such that $A(P) \cap A(T)=\phi$. If

$$
D \text { does not contain an }(s, t) \text { - ditrail with vertex set } V(T) \cup V(P) \text {, }
$$

then

$$
d_{V(T)}^{+}(y)+d_{V(T)}^{-}(x) \leq|V(T)|
$$

Proof By contradiction, we assume that $d_{V(T)}^{+}(y)+d_{V(T)}^{-}(x) \geq|V(T)|+1$. Throughout this proof we observe that for any vertex $w \in V(T)-\{x, y\}$,

$$
|(y, w),(w, x) \cap(A(D)-A(T))| \leq 1,
$$

otherwise, $T+(w, x)+P+(y, w)$ is a ditrail violating (5.2). We divide the proof to three cases.

Case (1) $\{x, y\} \cap V(T)=\emptyset$.

Since $d_{V(T)}^{+}(y)+d_{V(T)}^{-}(x) \geq|V(T)|+1$, then there must exist $w \in V(T)$ such that $(y, w),(w, x) \in$ $A(D)$ which contradicts observation (5.2).

Case (2) $\{x, y\} \subset V(T)$.

We divide $V(T)-\{x, y\}$ into the following disjoint sets observing that for every $v \in V(T)-\{x, y\}$, both $(v, x)$ and $(y, v)$ satisfy exactly one case of three cases, and considering observation (5.3):

$X_{m}=\{v \in V(T) \mid\{(v, x),(y, v)\} \subset A(T)\}$,

$X_{r}=\{v \in V(T) \mid(v, x) \in A(T)$ and $(y, v) \in A(D)-A(T)\}$,

$X_{l}=\{v \in V(T) \mid(v, x) \in A(D)-A(T)$ and $(y, v) \in A(T)\}$,

$Y_{r}=\{v \in V(T) \mid(y, v) \in A(T)$ and $(v, x) \notin A(D)\}$,

$Y_{l}=\{v \in V(T) \mid(y, v) \in A(D)-A(T)$ and $(v, x) \notin A(D)\}$,

$Z_{r}=\{v \in V(T) \mid(v, x) \in A(T)$ and $(y, v) \notin A(D)\}$,

$Z_{l}=\{v \in V(T) \mid(v, x) \in A(D)-A(T)$ and $(y, v) \notin A(D)\}$, 
$M=\{v \in V(T) \mid(v, x)$ and $(y, v) \notin A(D)\}$.

Subcase (2.1) $(y, x) \notin A(D)$

Since $d_{V(T)}^{+}(y)+d_{V(T)}^{-}(x) \geq|V(T)|+1,\{x, y\} \subset V(T)$ and $(y, x) \notin A(D)$, we have $\mid N_{V(T)}^{+}(y) \cap$ $N_{V(T)}^{-}(x) \mid \geq 3$ when $M=\phi$. Thus by observation (5.3) we have

$$
\left|X_{l} \cup X_{m} \cup X_{r}\right| \geq|M|+3
$$

By (5.4) and since $t$ may belong to $\{x\} \cup X_{l}$, then $\left|\partial_{T}^{+}(x)\right|+\left|\partial_{T}^{+}\left(X_{l}\right)\right| \geq|M|+2$. Since $\left|\partial_{T}^{+}(x)\right|+\left|\partial_{T}^{+}\left(X_{l}\right)\right| \geq|M|+2$ and by definition of $X_{m}, X_{l}$ and $Y_{r}$ there exists a ditrail $R \subseteq T$ starts at $\{x\} \cup X_{l}$ and terminates at $Y_{l} \cup X_{r} \cup\{y\}$ such that after removing the arcs of $R$, each vertex in $M \cup X_{m} \cup X_{l} \cup Y_{r}$ has positive degree in $T$ and $A(R) \cap\left(Z_{r}, x\right)_{T}=\emptyset$ (if $A(R) \cap\left(Z_{r}, x\right)_{T} \neq \emptyset$, then let $R$ start from last time $R$ visits $x$ ).

If $V(R) \cap Z_{l} \neq \emptyset$, then choose $u$ to be the last vertex from $V(R) \cap Z_{l}$ in $R$ and let $v$ be the first vertex from $Y_{l} \cup X_{r} \cup\{y\}$ in $R$. Therefore $T-R[u, v]+(u, x)+P+(y, v)$ or $(T-R[u, v]+(u, x)+P$ when $v=y$ ) is a ditrail contrary to (5.2).

Hence we may assume that $V(R) \cap Z_{l}=\emptyset$.

If $R$ starts at $x$ and terminates at $v \in Y_{l} \cup X_{r} \cup\{y\}$, then $T-R+P+(y, v)$ or $(T-R+P$ when $v=y$ ) violates (5.2).

If $R$ starts at $u \in X_{l}$ and terminates at $v \in Y_{l} \cup X_{r} \cup\{y\}$, then $T-R+(u, x)+P+(y, v)$ or $(T-R+(u, x)+P$ when $v=y)$ is a ditrail contradicting (5.2).

Subcase (2.2) $(y, x) \in A(D)$

$(y, x)$ must belong to $A(T)$, otherwise $T+(y, x)+P$ violates $(5.2)$. Since $d_{V(T)}^{+}(y)+d_{V(T)}^{-}(x) \geq$ $|V(T)|+1,\{x, y\} \subset V(T)$ and $(y, x) \in A(D)$, we have $\left|N_{V(T)}^{+}(y) \cap N_{V(T)}^{-}(x)\right| \geq 1$ when $M=\phi$. Thus by observation (5.3) and definition of $X_{l}, X_{m}$ and $X_{r}$ we have

$$
\left|X_{l} \cup X_{m} \cup X_{r}\right| \geq|M|+1
$$

Since $(y, x) \in A(T)$ and by (5.5) as well as since $t$ may belong to $\{x\} \cup X_{l}$, then $\left|\partial_{T}^{+}(x)\right|+$ $\left|\partial_{T}^{+}\left(X_{l}\right)\right| \geq|M|+1+1-1=|M|+1$. Since $\left|\partial_{T}^{+}(x)\right|+\left|\partial_{T}^{+}\left(X_{l}\right)\right| \geq|M|+1$ and by definition of $X_{m}, X_{l}$ and $Y_{r}$ there exists a ditrail $R \subseteq T$ starts at $\{x\} \cup X_{l}$ and terminates at $Y_{l} \cup X_{r} \cup\{y\}$ such that after removing the arcs of $R$, each vertex in $M \cup X_{m} \cup X_{l} \cup Y_{r}$ has positive degree in $T$ and $A(R) \cap\left(Z_{r}, x\right)_{T}=\emptyset$ (if $A(R) \cap\left(Z_{r}, x\right)_{T} \neq \emptyset$, then let $R$ start from last time $R$ visits $x$ ).

If $V(R) \cap Z_{l} \neq \emptyset$, then choose $u$ to be the last vertex from $V(R) \cap Z_{l}$ in $R$ and let $v$ be the first vertex from $Y_{l} \cup X_{r} \cup\{y\}$ in $R$. Therefore $T-R[u, v]+(u, x)+P+(y, v)$ or $(T-R[u, v]+(u, x)+P$ 
when $v=y$ ) is a ditrail contrary to (5.2).

Hence we may assume that $V(R) \cap Z_{l}=\emptyset$.

If $R$ starts at $x$ and terminates at $v \in Y_{l} \cup X_{r} \cup\{y\}$, then $T-R+P+(y, v)$ or $(T-R+P$ when $v=y$ ) violates (5.2).

If $R$ starts at $u \in X_{l}$ and terminates at $v \in Y_{l} \cup X_{r} \cup\{y\}$, then $T-R+(u, x)+P+(y, v)$ or $(T-R+(u, x)+P$ when $v=y)$ is a ditrail contradicting (5.2).

Case (3) $|\{x, y\} \cap V(T)|=1$.

Note that $(y, x) \notin A(D)$, otherwise $(y, x) \in A(D)-A(T)$ and we get contradiction with (5.2). Since $|\{x, y\} \cap V(T)|=1$ and $d_{V(T)}^{+}(y)+d_{V(T)}^{-}(x) \geq|V(T)|+1$ as well as $(x, y) \notin A(D)$, we have

$$
\left|N_{V(T)}^{+}(y) \cap N_{V(T)}^{-}(x)\right| \geq 2 \text { when } M=\phi .
$$

Subcase (3.1) $x \in V(T)$.

Since $y \notin V(T)$, we have $X_{m}=X_{l}=Y_{r}=\phi$. Therefore by (5.6), we have $\left|X_{r}\right| \geq|M|+2$. Since $\left|X_{r}\right| \geq|M|+2$ and since $t$ may equal $x$, we have that $\left|\partial_{T}^{+}(x)\right| \geq|M|+1$. The rest of the proof is the same idea of Subcase (2.1).

Subcase (3.2) $y \in V(T)$.

Since $x \notin V(T)$, we have $X_{m}=X_{r}=Z_{r}=\phi$. Therefore by (5.6), we have $\left|X_{l}\right| \geq|M|+2$. Since $\left|X_{l}\right| \geq|M|+2$ and since $t$ may belong to $X_{l}$, we have that $\left|\partial_{T}^{+}\left(X_{l}\right)\right| \geq|M|+1$. The rest of the proof is the same idea of Subcase (2.1).

Lemma (5.1.4) is sharp. Let $P$ be an $(x, y)$-dipath and $T$ be an $(s, t)$-dipath where $T=$ $s, z_{1} \ldots, z_{d}, z_{d+1}, \ldots z_{k}, t$ such that $V(P) \cap V(T)=\phi$. Let $D=P \cup T \cup\left(\cup_{i=1}^{d}\left\{\left(y, z_{i}\right)\right\}\right) \cup\left(\cup_{i=d+1}^{k}\left\{\left(z_{i}, x\right)\right\}\right) \cup$ $\{(y, s),(t, x)\}$. Any $(s, t)$-walk containing $V(T) \cup V(P)$ must use $\left(z_{d}, z_{d+1}\right)$ at least two times. Hence there is no any $(s, t)$-ditrail in $D$ containing $V(P) \cup V(T)$ eventhough $d_{V(T)}^{+}(y)+d_{V(T)}^{-}(x)=$ $|V(T)|$.

The following main theorem is almost an analogue of Theorem 5.1.3.

\subsection{Main Theorem}

Theorem 5.2.1. Let $D$ be a strong digraph. If

$$
d^{+}(x)+d^{+}(y)+d^{-}(u)+d^{-}(v) \geq 2 n-3
$$


for every pair $x, y$ of dominating non-adjacent vertices and every pair $u, v$ of dominated nonadjacent vertices, then $D$ is supereulerian.

Proof Since $D$ is strong, $D$ must have an eulerian subdigraph. Let $S$ be an eulerian subdigraph of $D$ such that among all eulerian subdigraphs of $D$

$$
|V(S)| \text { being maximized. }
$$

If $|V(S)|=|V(D)|$, then $S$ is a spanning eulerian subdigraph of $D$ and we are done. Assume by contradiction that $|V(D)|>|V(S)|>1$. Hence $V(D)-V(S) \neq \emptyset$. Since $D$ is strong and $S$ is a maximum eulerian subdigraph, there exists an $(S, S)$-dipath $Q$ on at least three vertices. Let $Q$ be chosen so that:

the length of a shortest dipath $P$ in $S$ between the endpoints of $Q$ is minimized.

Assume that $V(Q) \cap V(S)=\left\{z_{1}, z_{2}\right\}$, where $z_{1}, z_{2}$ are the first and the last vertex of $Q$. Assume that $u$ and $x$ are the first and the last vertex of $Q] z_{1}, z_{2}\left[\right.$, namely, $\left\{\left(z_{1}, u\right),\left(x, z_{2}\right)\right\} \subseteq A(Q)$. Note that $u$ may equal $x$. If $P=\left(z_{1}, z_{2}\right)$, then $S-\left(z_{1}, z_{2}\right)+A(Q)$ is an eulerian subdigraph with at least one more vertex than $S$, contrary to (5.8), moreover, by the maximality of $S, z_{1}$ cannot equal $z_{2}$. Therefore, $V(P) \geq 3$. Assume that $v$ and $y$ are the first and the last vertex of $\left.P\right] z_{1}, z_{2}[$, namely, $\left\{\left(z_{1}, v\right),\left(y, z_{2}\right)\right\} \subseteq A(P)$. Note that $v$ may equal $y$. (See Figure 1.5).

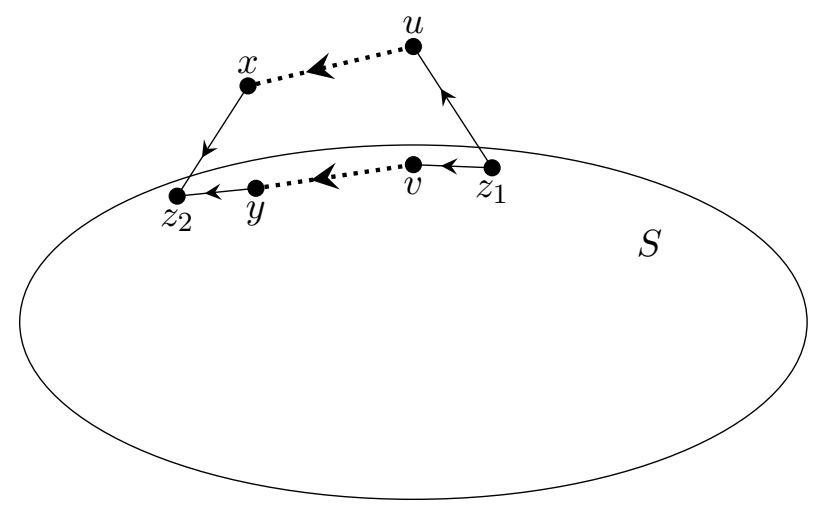

Figure 1.5.

Let $M=\left\{v, v_{1}, \ldots, v_{d-2}, y\right\}$, where $|M|=d$, be the set of vertices of $\left.P\right] z_{1}, z_{2}[$. By the minimality of $P$

$$
d_{M}(u)=d_{M}(x)=0
$$


So the pair $x, y$ is dominating non-adjacent vertices and the pair $u, v$ is dominated non-adjacent vertices. By the maximality of $S$, there is no vertex $w \in V(D)-V(S)$ such that $(y, w),(w, u) \in$ $A(D)$ or $(x, w),(w, v) \in A(D)$. Moreover, $d_{Q] z_{1}, z_{2}[}^{+}(y)=d_{Q] z_{1}, z_{2}[}^{-}(v)=0$ Therefore

$$
d_{V-V(S)}^{+}(x)+d_{V-V(S)}^{+}(y)+d_{V-V(S)}^{-}(u)+d_{V-V(S)}^{-}(v) \leq 2(n-s-1) .
$$

Let $S^{\prime}$ be the set of vertices of the $\left(z_{2}, z_{1}\right)$-ditrail obtained by removing from $S$ the arcs of $P$ and keeping only vertices left with positive degree in $S$, call this ditrail $R$. Let $H=S^{\prime} \cap M$. Then we have $\left|S^{\prime}\right|=s-d+h$, where $h=|H|<d$. By the maximality of $S$, equation (5.10) and lemma (5.1.4) applied to $R$ with $Q] z_{1}, z_{2}[$ and to $R$ with $P] z_{1}, z_{2}[$ we have

$$
\begin{gathered}
d_{V(S)}^{+}(x)+d_{V(S)}^{-}(u) \leq s-d+h \\
d_{S^{\prime}}^{+}(y)+d_{S^{\prime}}^{-}(v) \leq s-d+h
\end{gathered}
$$

If $y \notin H$, then $d_{V(S)-S^{\prime}}^{+}(y) \leq d-h-1$. If $y \in H$, then $\left(y, v^{\prime}\right) \notin A(D)$, where $v^{\prime}$ is the first vertex in $P] z_{1}, z_{2}$ [ not in $H$ (otherwise $\left\{\left(y, v^{\prime}\right) \cup P\left[v^{\prime}, y\right] \cup R \cup Q\right\}$ contradicts the maximality of $S$ ) and $d_{V(S)-S^{\prime}}^{+}(y) \leq d-h-1$. By the same way $d_{V(S)-S^{\prime}}^{-}(v) \leq d-h-1$. Thus

$$
d_{V(S)-S^{\prime}}^{+}(y)+d_{V(S)-S^{\prime}}^{-}(v) \leq 2(d-h-1)
$$

Putting together (5.11)-(5.14) we have

$$
d^{+}(x)+d^{+}(y)+d^{-}(u)+d^{-}(v) \leq 2(n-s-1)+2 s-2 d+2 h+2(d-h-1)
$$

Thus

$$
d^{+}(x)+d^{+}(y)+d^{-}(u)+d^{-}(v) \leq 2 n-4
$$

But, the pair $u, v$ is dominated non-adjacent and the pair $x, y$ is dominating non-adjacent, contradicting the hypothesis.

\subsection{Sharpness of the Main Theorem}

We show by the following example that the main result is sharp:

example. Let $G, H$ be two digraphs isomorphic to $K_{m}^{*}$, where $m \geq 2$. Let $u, x \in V(G)$ and $v, y \in V(H)$. Let $D_{m}=G \cup H \cup\left\{\left(z_{1}, u\right),\left(z_{1}, v\right),\left(x, z_{2}\right),\left(y, z_{2}\right),\left(z_{2}, z_{1}\right)\right\}$. Then $V\left(D_{m}\right)=n=$ $2 m+2$. (See Figure 5.2 for $m=3$ ). By Lemma 4.3.3 with $A=D\left[\left\{z_{1}\right\}\right], B_{1}=G$ and $B_{2}=H$, we conclude that $D_{m}$ is not supereulerian eventhough

$$
d^{+}(x)+d^{+}(y)+d^{-}(u)+d^{-}(v)=4 m=2 n-4
$$


Thus we have infinite family of nonsupereulerian digraphs which makes our main theorem sharp.

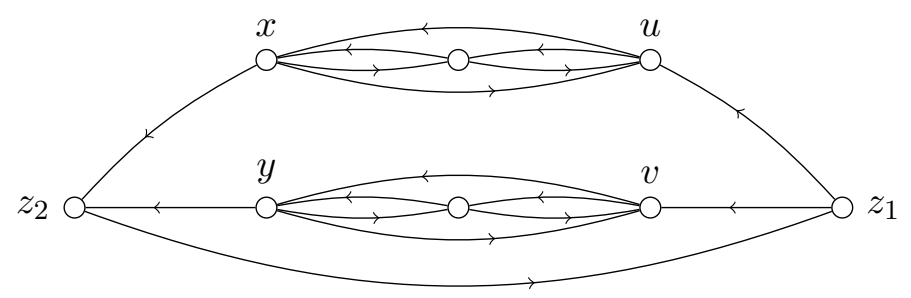

Figure 5.2. The digraph $D_{m}$.

\subsection{Future Tool}

In this section we present a proposition which is considered as a tool to help us to prove that some digraphs with certain specific conditions are supereulerian.

Proposition 5.4.1. Let $D$ be a strong simple digraph. If for every $(u, v) \in A(D)$ with $d^{+}(u) \geq 2$, one of the following holds:

(i) $N^{+}(u)-\{v\} \subseteq N^{+}(v)$.

(ii) For every $z \in N^{+}(u) \backslash\left(N^{+}(v) \cup\{v\}\right),\{(z, u),(z, v)\} \cap A(D) \neq \emptyset$.

(iii) For every ordered pairs $(a, b)$ in $N^{+}(u)$, there exists $c \in V(D)$ such that $\{(a, c),(c, a),[b, c]\} \subset$ $A(D)$ where $[b, c]$ is $(b, c)$ or $(c, b)$.

Then $D$ is supereulerian.

Proof. Since $D$ is strong, $D$ must have an eulerian subdigraph. Let $S$ be an eulerian subdigraph of $D$ such that among all eulerian subdigraphs of $D$

$$
|V(S)| \text { being maximized. }
$$

If $|V(S)|=|V(D)|$, then $S$ is a spanning eulerian subdigraph of $D$ and we are done. Assume by contradiction that $|V(D)|>|V(S)|>1$. Hence $V(D)-V(S) \neq \emptyset$. Since $D$ is strong and $S$ is a maximum eulerian subdigraph, there exists an $(S, S)$-dipath $Q$ on at least three vertices. Let $Q$ be chosen so that:

the length of a shortest dipath $P$ in $S$ between the endpoints of $Q$ is minimized.

Assume that $V(Q) \cap V(S)=\{x, y\}$ with $\left(x, v_{1}\right) \in A(Q)$. If $(x, y) \in A(S)$, then $S-(x, y)+A(Q)$ is an eulerian subdigraph with at least one more vertex than $S$, contrary to (5.15). Therefore, there must be a vertex $u_{1} \in V(P)-\{x, y\}$ such that $\left(x, u_{1}\right) \in A(P)$. (See Figure 5.1). 


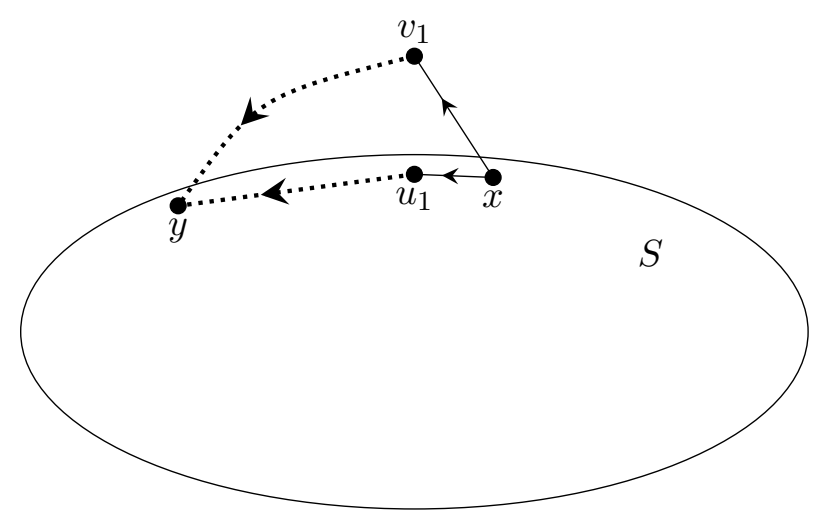

Figure 5.1.

(i) By contradiction, we assume that for every $(u, v) \in A(D)$ with $d^{+}(u) \geq 2$, then $N^{+}(u)-\{v\} \subseteq$ $N^{+}(v)$. Since $\left\{v_{1}, u_{1}\right\} \subseteq N^{+}(x)$, then $\left(x, u_{1}\right) \in A(D)$ with $d^{+}(x) \geq 2$. By our assumption $N^{+}(x)-\left\{u_{1}\right\} \subseteq N^{+}\left(u_{1}\right)$, so $\left(u_{1}, v_{1}\right) \in A(D)$. This implies that an $(S, S)$-dipath starts at $u_{1}$ and ends at $y$. Since the distance from $u_{1}$ to $y$ in $S$ is shorter than $P$, a contradiction to (5.16) is obtained.

(ii) By contradiction, we assume that for every $(u, v) \in A(D)$ with $d^{+}(u) \geq 2$, then for every $z \in N^{+}(u) \backslash\left(N^{+}(v) \cup\{v\}\right)$ we have $\{(z, u),(z, v)\} \cap A(D) \neq \emptyset$. Note that $v_{1} \notin N^{+}\left(u_{1}\right)$, otherwise by same argument in (i) we are done. By our assumption $\left\{\left(v_{1}, x\right),\left(v_{1}, u_{1}\right)\right\} \cap A(D) \neq \emptyset$. If $\left(v_{1}, x\right) \in A(D)$, then $S+\left\{\left(v_{1}, x\right),\left(x, v_{1}\right)\right\}$ is an eulerian subdigraph of $D$ with more vertices than $S$, contrary to (5.15). If $\left(v_{1}, u_{1}\right) \in A(D)$, then $S+\left\{\left(x, v_{1}\right),\left(v_{1}, u_{1}\right)\right\}-\left\{\left(x, u_{1}\right)\right\}$ is an eulerian subdigraph of $D$ with more vertices than $S$, contrary to (5.15). This proves (ii).

(iii) By contradiction, we assume that for every $(u, v) \in A(D)$ with $d^{+}(u) \geq 2$, then for every ordered pairs $(a, b)$ in $N^{+}(u)$ there exists $c \in V(D)$ such that $\{(a, c),(c, a),[b, c]\} \subset A(D)$. Consider the ordered pairs $\left(v_{1}, u_{1}\right)$. By our assumption there exists a vertex $c \in V(D)$ such that $\left\{\left(v_{1}, c\right),\left(c, v_{1}\right),\left[u_{1}, c\right]\right\} \subset A(D)$. If $c \in V(S)$, then $S+\left\{\left(v_{1}, c\right),\left(c, v_{1}\right)\right\}$ is an eulerian subdigraph of $D$ with more vertices than $S$, contrary to (5.15). If $c \in V(D)-V(S)$, we have four cases:

(A) $\left[u_{1}, c\right]=\left(u_{1}, c\right)$ and $c \notin V(Q)$. Then the dipath $\left\{\left(u_{1}, c\right),\left(c, v_{1}\right)\right\}+Q\left[v_{1}, y\right]$ is an $(S, S)-\operatorname{dipath}$ and the distance from $u_{1}$ to $y$ in $S$ is shorter than $P$, a contradiction to (5.16).

(B) $\left[u_{1}, c\right]=\left(u_{1}, c\right)$ and $c \in V(Q)$. Then the dipath $\left\{\left(u_{1}, c\right)\right\}+Q[c, y]$ is an $(S, S)-\operatorname{dipath}$ and same as for $(\mathrm{A})$. 
(C) $\left[u_{1}, c\right]=\left(c, u_{1}\right)$ and $c \notin V(Q)$. Then $S+\left\{\left(x, v_{1}\right),\left(v_{1}, c\right),\left(c, u_{1}\right)\right\}-\left\{\left(x, u_{1}\right)\right\}$ is an eulerian subdigraph of $D$ with more vertices than $S$, contrary to $(5.15)$.

(D) $\left[u_{1}, c\right]=\left(c, u_{1}\right)$ and $c \in V(Q)$. Then $S+Q[x, c]+\left\{c, u_{1}\right\}-\left\{\left(x, u_{1}\right)\right\}$ is an eulerian subdigraph of $D$ with more vertices than $S$, contrary to (5.15). This proves (iii). 


\section{Chapter 6}

\section{Matching and Minimum Degree in Graph}

\subsection{Introduction}

We consider finite and simple graphs. Usually, we use $G$ to denote a graph. Undefined terms and notations will follow [8]. In particular, $\kappa(G), \kappa^{\prime}(G), \alpha(G)$ and $\alpha^{\prime}(G)$ denote the connectivity, the edge connectivity, the independence number, and the matching number of a graph $G$, respectively. Throughout this chapter, we use the notation $u v$ to denote an edge between $u$ and $v$ in a graph. When $u v \in E(G)$, we say that $u$ and $v$ are adjacent. If two edges of $G$ have a common vertex, we say that these two edges are adjacent in $G$.

For graphs $H$ and $G$, by $H \subseteq G$ we mean that $H$ is a subgraph of $G$. Following [8], for a graph $G$ with $X, Y \subseteq V(G)$, define

$$
(X, Y)_{G}=\{x y \in E(G): x \in X, y \in Y\} .
$$

When $Y=V(G)-X$, we define

$$
\partial_{G}(X)=(X, V(G)-X)_{G}
$$

For a vertex $v \in V(G), d_{G}(v)=\left|\partial_{G}(\{v\})\right|$ is the degree of $v$ in $G$. Finally, we define the following notation: $\delta(G)=\min \left\{d_{G}(v): v \in V(G)\right\}$.

When the graph $G$ is understood from the context, we often omit the subscript $G$. By the definition of $\kappa^{\prime}(G)$ in [8], we note that for any integer $k \geq 0$ and a graph $G$,

$$
\kappa^{\prime}(G) \geq k \text { if and only if for any nonempty proper subset } X \subset V(G),\left|\partial_{G}(X)\right| \geq k .
$$


A graph $G$ is eulerian if $G$ is connected and for any $v \in V(G), d(v)$ is even. A graph $G$ is supereulerian if $G$ contains a spanning eulerian subgraph. A graph $G$ is a closed trail if it is eulerian.

A well known theorem of Chvátal and Erdös [12] states that if $|V(G)| \geq 3$ and if $\kappa(G) \geq$ $\alpha(G)$, then $G$ is hamiltonian.

Moreover, there have been investigations on supereulerian properties of a graph $G$ with given inequality constraints on $\kappa^{\prime}(G), \alpha(G)$ and $\alpha^{\prime}(G)$, as seen in [1], [15], [18], [22] and [23], among others. In [5], Bang-Jensen and Maddaloni proved that if $\kappa^{\prime}(G) \geq \alpha(G)$ for a graph $G$, then $G$ is supereulerian. This motivates us to present and prove the main result of this chapter.

In the next section, we present some of the former theorems and develop a few lemmas which will be used in our arguments. The main result will be given in the third section.

\subsection{Tools}

In this section, we present some tools needed in our arguments. Let $M$ be a matching in a graph $G$. We use $V(M)$ to denote the set of vertices in $G$ that are incident with an edge in $M$. A path $P$ is an $M$-augmenting path if the edges of $P$ are alternately in $M$ and in $E(G)-M$, and if both end vertices of $P$ are not in $V(M)$. The following theorem is fundamental.

Theorem 6.2.1. (Berge, [6]) A matching $M$ in $G$ is a maximum matching if and only if $G$ does not have $M$-augmenting paths.

The next two Theorems will also be needed in our proofs.

Theorem 6.2.2. (Jaeger and Catlin [10]) Every 4-edge-connected graph is supereulerian.

Theorem 6.2.3. (Bang-Jensen and Maddaloni [5]) Let $G$ be a graph on at least three vertices. If $\kappa^{\prime}(G) \geq \alpha(G)$, then $G$ is supereulerian.

Two more lemmas will also be needed. Throughout the rest of this section, $G$ always denotes a graph.

Lemma 6.2.4. Let $k>0$ be an integer and $G$ be a graph with a matching $M$ such that $|M|=k$. Suppose that $V(G)-V(M)$ has a subset $X$ with $|X| \geq 2$ such that for any $v \in X, d(v) \geq k$. If $X$ has at least one vertex $u$ such that $d(u) \geq k+1$, then there exists a matching $M^{\prime}$ in $G$ such that $|M|<\left|M^{\prime}\right|$. 
Proof. By contradiction, we assume that $M$ is a maximum matching in $G$. By Theorem 6.2.1, $G$ has no $M$-augmenting path. Let $u, v \in X$ be distinct vertices such that $d(u) \geq k+1$. Since $M$ is maximum, $u$ and $v$ are not adjacent in $G$, and so any vertices adjacent to $u$ or $v$ must be in $V(M)$. Since $G$ has no $M$-augmenting path, we have the following observations.

(A) For each edge $e=x y \in M$, exactly one in $\{u x, v y\}$ can be in $E(G)$, and exactly one in $\{u y, v x\}$ can be in $E(G)$. If not, by symmetry, we may assume that $u x, v y \in E(G)$, and so $\{u x, x y, v y\}$ induces an $M$-augmenting path in $G$.

(B) From Observation (A), for each edge $e=x y \in M$, if $u x \in E(G)$ and $u y \in E(G)$, then $v x, v y \notin E(G)$, and so $\left|(\{u, v\},\{x, y\})_{G}\right| \leq 2$.

(C) From Observation (A), for each edge $e=x y \in M$, if $u x \in E(G)$ and $v x \in E(G)$, then $u y, v y \notin E(G)$, and so $\left|(\{u, v\},\{x, y\})_{G}\right| \leq 2$.

(D) From Observation (A), for each edge $e=x y \in M$, if $v x \in E(G)$ and $v y \in E(G)$, then $u x, u y \notin E(G)$, and so $\left|(\{u, v\},\{x, y\})_{G}\right| \leq 2$.

It follows from Observations (B), (C) and (D) that $|\partial(u) \cup \partial(v)| \leq 2|M|$. As $d(v) \geq k$ and $d(u) \geq k+1$, we have

$$
2 k+1=k+(k+1) \leq d(v)+d(u)=|\partial(v) \cup \partial(u)| \leq 2|M|=2 k .
$$

a contradiction. This proves the lemma.

Corollary 6.2.5. Let $G$ be a graph with $\alpha^{\prime}(G)=k$. If $\kappa^{\prime}(G) \geq \alpha^{\prime}(G)$, then

i) $\kappa^{\prime}(G)=\alpha^{\prime}(G)$ when $|V(G)| \geq 2 k+2$.

ii) $\kappa^{\prime}(G) \leq 2 \alpha^{\prime}(G)$ when $2 k \leq|V(G)| \leq 2 k+1$.

Proof. i) By contradiction, we assume that $\kappa^{\prime}(G) \geq k+1$. Let $M$ denote a maximum matching of $G$. Then $|V(G)-V(M)| \geq 2 k+2-2 k=2$. Since $\kappa^{\prime}(G) \geq k+1$, for every vertex $u \in V(G)-V(M), d(u) \geq k+1$. It follows by Lemma 6.2 .4 that $M$ is not a maximum matching of $G$, and so a contradiction obtains.

ii) Since $2 k \leq|V(G)| \leq 2 k+1$, then $\forall v \in V(G), 2 k \geq d(v) \geq \kappa^{\prime}(G)$. Thus, $\kappa^{\prime}(G) \leq 2 \alpha^{\prime}(G)$.

Lemma 6.2.6. Let $k>0$ be an integer, $G$ be a graph on $n \geq 2 k+2$ vertices and $M$ be a maximum matching of $G$ with $|M|=k$. Suppose that

$$
d(v) \geq k, \text { for every vertex } v \in V(G) .
$$

Let $X=V(G)-V(M)$. Then

$$
\text { for every } x \in X \text {, we have } d(x)=k \text {. }
$$


Moreover, if $n \geq 2 k+3$ or if $n=2 k+2$ and $G$ is connected, then for every $e=u v \in M$ and for every $x \in X$, we have the following conclusions.

(i) There exists exactly one $v(e) \in\{u, v\}$ such that $v(e) x$ is in $E(G)$, and the vertex $u(e) \in$ $\{u, v\}-\{v(e)\}$ is not adjacent to any vertex in $X$.

(ii) The set $\{u(e): e \in M\}$ is an independent set in $G$ such that $d(u(e))=k$ for any $e \in M$ and such that for any $e, e^{\prime} \in M, u(e) v\left(e^{\prime}\right), v(e) u\left(e^{\prime}\right) \in E(G)$.

Proof. Let $N(X)$ denote the set of vertices in $G$ that is adjacent to a vertex in $X$. Since $M$ is a maximum matching, by Theorem 6.2.1

$$
G \text { does not have an } M \text {-augmenting path. }
$$

By 6.4, we have $N(X) \subseteq V(M)$. Since $n \geq 2 k+2$ and $|M|=k$, we have $|X| \geq 2$. Since $M$ is a maximum matching, it follows by (6.2) and by Lemma 6.2.4 that (6.3) must hold. In the rest of the proof for this lemma, we consider two cases.

Case 1. $G$ is a graph with $n=|V(G)| \geq 2 k+3$.

Let $m=|X|$. Then $m \geq 3$. By $(6.3)$,

$$
\left|(X, V(M))_{G}\right|=m k .
$$

We further observed that, by (6.4),

for any $e \in M$, the vertices of $e$ are incident with at most $m$ edges in $(X, V(M))_{G}$.

Let $e=u v \in M$. If $u, v \in N(X)$, then by (6.4), there must be a unique $x \in X$ such that $x$ is adjacent to both $u$ and $v$; and for any $x^{\prime} \in X-\{x\}, x^{\prime}$ is not adjacent to either $u$ nor $v$. By (6.3), $x$ is adjacent to $k$ vertices in $V(M)$. As $\left|(\{x\},\{u, v\})_{G}\right|=2$, it follows by $(6.5)$ and $m \geq 3$ that

$$
\left|(X, V(M)-\{u, v\})_{G}\right|=m k-2 \geq m k-m+1=m(k-1)+1 .
$$

If $k>1$, then there must be an edge $a \in M-\{e\}$ such that the vertices of $a$ are incident with at least $m+1$ edges in $(X, V(M))_{G}$, contrary to (6.6). Hence in this case we must have $k=1$ and $M=\{e\}$. As $|X| \geq 3$, it follows from (6.5) with $m \geq 3$ that $G$ must have an $M$-augmenting path, contrary to (6.4). Hence for any $e=u v \in M$, exactly one of $u$ or $v$ is adjacent to vertices in $X$.

Thus for each $e \in M$, let $v(e)$ denote the unique vertex of $e$ that is adjacent to vertices in $X$ and $u(e)$ the other vertex of $e$ which is not adjacent to any vertex in $X$. Then as $|X|=m$, 
$\left|(v(e), X)_{G}\right| \leq m$. It follows from (6.5) that

$$
k m \geq \sum_{e \in M}\left|(v(e), X)_{G}\right|=\left|(V(M), X)_{G}\right|=m k .
$$

This implies that for each $x \in X$ and for each $e \in M$, both $v(e) x$ is in $E(G)$. This proves (i) for Case 1.

Let $Y=\{u(e): e \in M\}$ and we shall show that $Y$ is an independent set. In fact, if for some $e_{1}, e_{2} \in M, u\left(e_{1}\right) u\left(e_{2}\right) \in E(G)$, then for any distinct $x_{1}, x_{2} \in X,\left\{x_{1} v\left(e_{1}\right), e_{1}, u\left(e_{1}\right) u\left(e_{2}\right)\right.$, $\left.e_{2}, x_{2} v\left(e_{2}\right)\right\}$ induces an $M$-augmenting path, contrary to (6.4). Thus each $u(e) \in Y$ can only be adjacent to vertices in $\{v(e): e \in M\}$. As $|\{v(e): e \in M\}|=k$, by (6.2), we conclude that for each $e \in M, d(u(e))=k$, and for any $e, e^{\prime} \in M, u(e) v\left(e^{\prime}\right), v(e) u\left(e^{\prime}\right) \in E(G)$. This proves (ii) for Case 1.

Case 2. $\quad G$ is connected and $|V(G)|=2 k+2$.

Then $X=\{w, z\}$. Let $M=\left\{e_{1}, \cdots, e_{k}\right\}$. Let $M_{w} \subseteq M$ denote the edges in $M$ each of which has a vertex adjacent to $w$. We define $M_{z}$ similarly. By (6.3), $\left|M_{w}\right| \geq \frac{k}{2}$ and $\left|M_{z}\right| \geq \frac{k}{2}$.

Subcase 1. $\left|M_{w}\right|=\frac{k}{2}$ or $\left|M_{z}\right|=\frac{k}{2}$.

Note that in this case, $k$ must be even. We assume, without loss of generality, that $M_{w}=$ $\left\{e_{1}, \cdots, e_{\frac{k}{2}}\right\}$. By (6.4), we must have $M_{z}=\left\{e_{\frac{k}{2}+1}, \cdots, e_{k}\right\}$. Again by (6.4), for each $x \in V\left(M_{w}\right)$ and $y \in V\left(M_{z}\right)$, we conclude that $x y \notin E(G)$. Thus

$$
\left(V\left(M_{w}\right) \cup\{w\}, V\left(M_{z}\right) \cup\{z\}\right)_{G}=\emptyset,
$$

contrary to the assumption that $G$ is connected. This shows that Subcase 1 cannot occur.

Subcase 2. $\left|M_{w}\right|>\frac{k}{2}$ and $\left|M_{z}\right|>\frac{k}{2}$.

Therefore, $M_{w} \cap M_{z} \neq \emptyset$. Note that by (6.4), if an edge $e \in M$ whose vertices are adjacent to both $w$ and $z$, then exactly one vertex of $e$ can be adjacent to both $w$ and $z$. Let $M^{\prime}=M_{w} \cap M_{z}=$ $\left\{e_{i}^{\prime}=x_{i} y_{i},(i=1, \ldots, d ; 1 \leq d \leq k)\right\} \subseteq M$. Without loss of generality, we assume that each $e_{i}^{\prime}$ has a unique vertex $x_{i}$ with $x_{i} w, x_{i} z \in E(G)$. Let $M^{\prime \prime}=M-M^{\prime}=\left\{e_{j}^{\prime \prime}=r_{j} s_{j},(j=1, \ldots, k-d)\right\}$. We justify the following observations.

(A) By (6.4), for each $y_{i}, y_{i} w, y_{i} z \notin E(G)$.

(B) The set $\left\{y_{1}, y_{2}, \cdots, y_{d}\right\}$ must be an independent set. This is warranted by (6.4).

(C) Suppose that Lemma 6.2.6(i) or (ii) does not hold. Then $d \leq k-1$.

In fact, if $d=k$, then $M=M^{\prime}$ and each $x_{i}$ is adjacent to both $w$ and $z$. By (A), for each 
$e_{i}=x_{i} y_{i} \in M$, we must have $y_{i} w, y_{i} z \notin E(G)$. Hence Lemma 6.2.6(i) must hold. Furthermore, by Observations (A) and (B), each $y_{i}$ can only be adjacent to $\left\{x_{1}, x_{2}, \cdots, x_{d}\right\}$. By (6.2), for any $i$, we must have $d\left(y_{i}\right)=k$, and for any $1 \leq i, i^{\prime} \leq k$, we must have $x_{i} y_{i^{\prime}} \in E(G)$. Hence Lemma 6.2.6(ii) holds as well.

(D) From Observation (A), for each $e=x_{i} y_{i} \in M^{\prime},\left|\left(\left\{x_{i}, y_{i}\right\},\{w, z\}\right)_{G}\right|=2$.

(E) For each $j$ with $1 \leq j \leq k-d$, there exists exactly one vertex in $\{w, z\}$ which is adjacent to both $r_{j}$ and $s_{j}$.

By the definition of $M^{\prime \prime}$, for each $j$ with $1 \leq j \leq k-d$, there exists at most one vertex in $\{w, z\}$ which is adjacent to both $r_{j}$ and $s_{j}$. By contradiction, we assume that $r_{1} s_{1} \in M^{\prime \prime}$ with $\left|\left(\{w, z\},\left\{r_{1}, s_{1}\right\}\right)_{G}\right| \leq 1$. For any other $r_{j} s_{j} \in M^{\prime \prime}$ with $j \geq 2$, we have $\left|\left(\{w, z\},\left\{r_{j}, s_{j}\right\}\right)_{G}\right| \leq 2$. It follows from (6.3) and Observation (D) that

$$
\begin{aligned}
2 k= & \left|(\{w, z\}, V(M))_{G}\right| \\
= & \left|\left(\{w, z\}, V\left(M^{\prime}\right)\right)_{G}\right|+\left|\left(\{w, z\},\left\{r_{1}, s_{1}\right\}\right)_{G}\right| \\
& +\sum_{j=2}^{k-d}\left|\left(\{w, z\},\left\{r_{j}, s_{j}\right\}\right)_{G}\right| \leq 2 d+1+2(k-d-1)=2 k-1<2 k,
\end{aligned}
$$

a contradiction. This justifies Observation (E).

(F) For any $x_{i} y_{i} \in M^{\prime}$ and for any $r_{j} s_{j} \in M^{\prime \prime}, y_{i} r_{j}, y_{i} s_{j} \notin E(G)$. In fact, if $y_{i} r_{j} \in E(G)$, then by Observation (E), we may assume that $r_{j} s_{j} \in M_{w}$, and so $\left\{w s_{j}, r_{j} s_{j}, r_{j} y_{i}\right.$,

$\left.x_{i} y_{i}, z x_{i}\right\}$ will induce an $M$-augmenting path, contrary to (6.4), (same argument when $y_{i} s_{j} \in$ $E(G))$. This justifies $(\mathrm{F})$.

We argue by contradiction to prove (i) and (ii). As $M^{\prime}=M_{w} \cap M_{z} \neq \emptyset, d \geq 1$ and so $y_{1}$ exists. By Observations (A), (B) and (F), $y_{1}$ can only be adjacent to $\left\{x_{1}, x_{2}, \cdots, x_{d}\right\}$. Hence $d\left(y_{1}\right) \leq\left|\left\{x_{1}, x_{2}, \cdots, x_{d}\right\}\right|=d$. By $(6.2)$ and by Observation $(\mathrm{C})$, that $k \leq d\left(y_{1}\right) \leq d \leq(k-1)$, a contradiction. This implies that we must have $d=k$, and so by Observation (C), this proves the lemma for Case 2.

Theorem 6.2.7. Let $k>0$ be an integer, $G$ be a graph on $n \geq 2 k$ vertices with $\alpha^{\prime}(G)=k$. Suppose that if $n=2 k+2$, then $G$ is connected. If

$$
\delta(G) \geq k, \text { then } \kappa^{\prime}(G) \geq k \text {. In particular } \kappa^{\prime}(G)=k \text { when } n \geq 2 k+2 .
$$

Proof Let $M$ be a matching of maximum size of $G$. We proceed our proof in the following cases.

Case 1. $2 k \leq n \leq 2 k+1$. 
Let $X$ be an arbitrary nonempty proper subset of $V(G)$, and let $Y=V(G)-X$. Since $|X|+|Y|=n \leq 2 k+1$, either $1 \leq|X| \leq k$ or $1 \leq|Y| \leq k$. By symmetry, we may assume that $1 \leq|X|=m \leq k$. By (6.7), for each $x \in X .\left|(\{x\}, Y)_{G}\right| \geq k-(m-1)$. Thus $\left|\partial_{G}(X)\right| \geq$ $m(k-(m-1))=-m^{2}+m(k+1)$. As this is a quadratic function with $1 \leq m \leq k$, it follows that $\left|\partial_{G}(X)\right| \geq-m^{2}+m(k+1) \geq k$, and so by $(6.1), \kappa^{\prime}(G) \geq k$. This proves Case 1 .

Case 2. $n \geq 2 k+2$.

Let $X$ satisfying $\emptyset \neq X \subset V(G)$ be an arbitrary nonempty proper vertex subset. We are to prove $\left|\partial_{G}(X)\right| \geq k$.

Let $Z=V(G)-V(M)$. By Lemma 6.2.6 (i), for any $e=u v \in M$, there exists a unique $v(e) \in\{u, v\}$ such that for any $z \in Z, v(e) z \in E(G)$. Let $M_{v}=\{v(e): e \in M\}$, and $M_{u}=V(M)-M_{v}$. Let $m \geq 2$ be the integer satisfying $n=2 k+m$. By Lemma 6.2.6, for each $v \in M_{v}$, and for any $u \in Z \cup M_{u}, v u \in E(G)$. We consider the following cases.

Subcase 2.1. $\quad\left(M_{u} \cup Z\right) \subseteq X$ (or $\left.\left(M_{u} \cup Z\right) \cap X=\emptyset\right)$. We assume that $M_{u} \cup Z \subseteq X$ as by symmetry, the proof for $\left(M_{u} \cup Z\right) \cap X=\emptyset$ is similar. As $V(G)=M_{u} \cup M_{v} \cup Z$, there exists a $y \in M_{v}-X \subseteq V(G)-X$. By Lemma 6.2.6, $\left|\partial_{G}(X)\right| \geq\left|\left(M_{u} \cup Z,\{y\}\right)_{G}\right|=|Z|+\left|M_{u}\right|=k+m>k$.

Subcase 2.2. $M_{v} \subseteq X$ (or $M_{v} \subseteq V(G)-X$ ). We assume that $M_{v} \subseteq X$, as by symmetry, the proof for $M_{v} \subseteq V(G)-X$ is similar. Then $M_{u} \cup Z-X \neq \emptyset$. Pick $y \in M_{u} \cup Z-X$. Then by Lemma 6.2.6, $\left|\partial_{G}(X)\right| \geq\left|\left(M_{v},\{y\}\right)_{G}\right|=\left|M_{v}\right|=k$.

Subcase 2.3. Both $M_{u} \cup Z-X \neq \emptyset$ and $X \cap\left(M_{u} \cup Z\right) \neq \emptyset$, and both $M_{v}-X \neq \emptyset$ and $X \cap M_{v} \neq \emptyset$.

Pick $x \in X \cap\left(M_{u} \cup Z\right)$ and $y \in\left(M_{u} \cup Z\right)-X$. Then by Lemma 6.2.6,

$$
\left|\partial_{G}(X)\right| \geq\left|\left(\{x\}, M_{v}-X\right)_{G}\right|+\left|\left(M_{v} \cap X,\{y\}\right)_{G}\right|=\left|M_{v}-X\right|+\left|M_{v} \cap X\right|=\left|M_{v}\right|=k .
$$

It follows that we always have $\left|\partial_{G}(X)\right| \geq k$, and so $\kappa^{\prime}(G) \geq k$. By corollary $6.2 .5, \kappa^{\prime}(G)=k$. This proves Case 2 .

\subsection{The Main Result}

Theorem 6.3.1. Let $k$ be an integer such that $k \geq 2$ when $|V(G)|$ is even, otherwise $k \geq 3$. Let $G$ be a graph on $n \geq 2 k$ vertices with $\alpha^{\prime}(G)=k$. Suppose that if $n=2 k+2$, then $G$ is 
connected.

$$
\text { If } \delta(G) \geq \alpha^{\prime}(G) \text {, then } G \text { is supereulerian. }
$$

Proof Let $m=n-2 k$. We proceed our proof in the following cases.

Case 1. $\alpha^{\prime}(G) \geq 4$.

By Theorem (6.2.7), $\kappa^{\prime}(G) \geq \alpha^{\prime}(G) \geq 4$. By Jaeger and Catlin Theorem (6.2.2), $G$ is supereulerian.

Case 2. $\alpha^{\prime}(G)=3$ and $m \geq 2$.

By Lemma 6.2.6(i), for any $e_{i}=u_{i} v_{i} \in M, i \in\{1,2,3\}$ where $M$ is a maximum matching, there exists a unique $v\left(e_{i}\right) \in\left\{u_{i}, v_{i}\right\}$ such that for any $x \in V(G)-V(M), v\left(e_{i}\right) x \in E(G)$. Let $M_{v}=\left\{v\left(e_{i}\right): e_{i} \in M\right\}=\left\{v_{1}, v_{2}, v_{3}\right\}$ and $M_{u}=V(M)-M_{v}=\left\{u_{1}, u_{2}, u_{3}\right\}$. It follows by Lemma 6.2.6(ii) that there exists a subgraph $H \subseteq G$ with $H \cong K_{3,3}$ and $H \subseteq G\left[M_{v} \cup M_{u}\right]$.

Subcase 2.1. $m$ is odd integer.

Let $d \geq 1$ be the integer satisfying $n=2 k+2 d+1$. Let $V(G)-M_{v} \cup M_{u}=\left\{x_{1}, y_{1}, x_{2}, y_{2}, \ldots, x_{d}, y_{d}, z\right\}$. Then using $H$ and by Lemma 6.2.6(i), the subgraph $T \subseteq G$ with $E(T)=\left\{v_{1} u_{1}, u_{1} v_{2}, v_{2} u_{2}\right.$, $\left.u_{2} v_{3}, v_{3} u_{3}, u_{3} v_{2}, v_{2} z, z v_{1}\right\} \cup\left(\cup_{j=1}^{d}\left\{v_{1} x_{j}, x_{j} v_{2}, v_{2} y_{j}, y_{j} v_{1}\right\}\right)$ is a spanning closed trail in $G$. Thus $G$ is supereulerian.

Subcase 2.2. $m$ is even integer.

Let $d \geq 1$ be the integer satisfying $n=2 k+2 d$. Let $V(G)-M_{v} \cup M_{u}=\left\{x_{1}, y_{1}, x_{2}, y_{2}, \ldots, x_{d}, y_{d}\right\}$. Then using $H$ and by Lemma 6.2.6(i), the subgraph $T \subseteq G$ with $E(T)=\left\{v_{1} u_{1}, u_{1} v_{2}, v_{2} u_{2}\right.$, $\left.u_{2} v_{3}, v_{3} u_{3}, u_{3} v_{1}\right\} \cup\left(\cup_{j=1}^{d}\left\{v_{1} x_{j}, x_{j} v_{3}, v_{3} y_{j}, y_{j} v_{1}\right\}\right)$ is a spanning closed trail in $G$. Thus $G$ is supereulerian.

Case 3. $\alpha^{\prime}=3$ and $m \leq 1$.

By Theorem (6.2.7), $\kappa^{\prime}(G) \geq 3$. If $m=0$, then $\alpha(G) \leq 3$. Thus by applying Theorem (6.2.3) we get the desired result. If $m=1$, then $\alpha(G) \leq 4$. When $\alpha(G) \leq 3$, then by applying Theorem (6.2.3) we get the desired result. When $\alpha(G)=4$, then let $M$ be a maximum matching in $G$ with $M=\left\{u_{1} v_{1}, u_{2} v_{2}, u_{3} v_{3}\right\}$ and $V(G)-V(M)=\{x\}$. Since $\alpha(G)=4$, then by symmetry we may assume that $X=\left\{v_{1}, v_{2}, v_{3}, x\right\}$ is the independent set in $G$. Since $\delta(G) \geq \alpha^{\prime}(G)=3$, then $E(G)=\cup_{i=1}^{3}\left\{x u_{i}, v_{1} u_{i}, v_{2} u_{i}, v_{3} u_{i}\right\}$. Therefore the subgraph $T \subseteq G$ with $E(T)=\left\{x u_{1}, u_{1} v_{2}, v_{2} u_{3}, u_{3} v_{3}, v_{3} u_{1}, u_{1} v_{1}, v_{1} u_{2}, u_{2} x\right\}$ is a spanning closed trail in $G$. Thus $G$ is supereulerian. 
Case 4. $\alpha^{\prime}(G)=2$ and $n$ is even.

When $m \geq 2$, then the proof is similar of the proof in Subcase 2.2. When $m=0$, then the proof is similar of the proof when $m=0$ in Case 3 .

\subsection{Sharpness of the Main Result}

Theorem (6.3.1 ) is sharp and the following example show that:

Example 1. For each integer $t \geq 1$ let $G_{t}$ be a graph with $V\left(G_{t}\right)=\left\{v_{1}, v_{2}, v_{3}, u_{1}, u_{2}, u_{3}, x_{1}, x_{2}, \ldots, x_{t}\right\}$ and $E\left(G_{t}\right)=\left\{v_{1} u_{1}, u_{1} v_{3}, v_{3} u_{3}, u_{3} v_{1}, v_{2} u_{2}, u_{2} v_{3}\right\} \cup\left(\cup_{j=1}^{t}\left\{v_{1} x_{j}, x_{j} v_{2}\right\}\right)$. Then $\left\{G_{t}\right\}_{t \geq 1}$ is an infinite family of connected graphs with $\delta(G)=2$ and $\alpha^{\prime}(G)=3$ and $G_{t}$ is not supereulerian. Because otherwise there exists a spanning closed trail $S$ in $G_{t}$ with $\left\{u_{1} v_{3}, u_{2} v_{3}, u_{3} v_{3}\right\} \subset E(S)$. However, $d_{G_{t}}\left(v_{3}\right)=3$ which implies that $d_{S}\left(v_{3}\right)=3$, contradiction with $S$ is even.(See Figure $6.1)$.

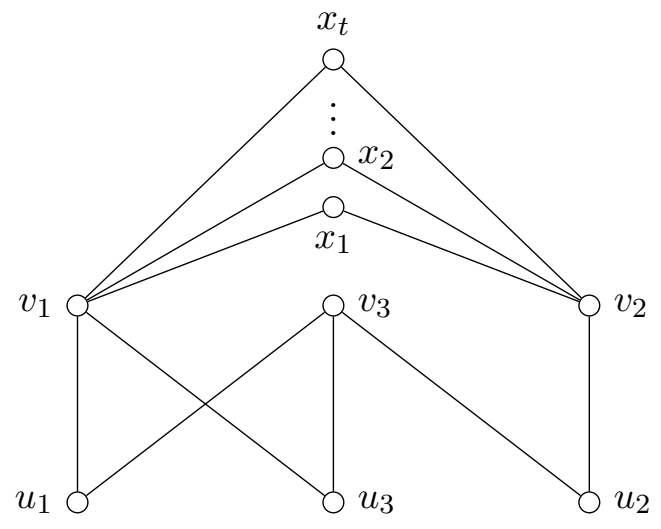

Figure 6.1. The graph $G_{t}$.

When $\delta(G)=\alpha^{\prime}(G)=2$ and $n$ is odd we have infinite family of non-supereulerian graphs as following:

Example 2. For each odd integer $s \geq 1$ let $G_{s}$ be a graph with $V\left(G_{s}\right)=\left\{v_{1}, v_{2}, u_{1}, u_{2}, x_{1}, x_{2}, \ldots, x_{s}\right\}$ and $E\left(G_{s}\right)=\left\{v_{1} u_{1}, v_{2} u_{2}, v_{1} u_{2}, v_{2} u_{1}\right\} \cup\left(\cup_{j=1}^{s}\left\{v_{1} x_{j}, x_{j} v_{2}\right\}\right)$. Then $\left\{G_{s}\right\}_{s \geq 1}$ is an infinite family of graphs with $\delta(G)=2$ and $\alpha^{\prime}(G)=2$ and $G_{s}$ is not supereulerian. Because otherwise there exists a spanning closed trail $S$ in $G_{s}$ with $\left\{u_{1} v_{1}, u_{2} v_{1}\right\} \cup\left(\cup_{j=1}^{s}\left\{v_{1} x_{j}\right\}\right) \subset E(S)$. However, $d_{G_{s}}\left(v_{1}\right)=s+2$ which implies that $d_{S}\left(v_{1}\right)=s+2$, contradiction with $S$ is even.(See Figure 6.2). 


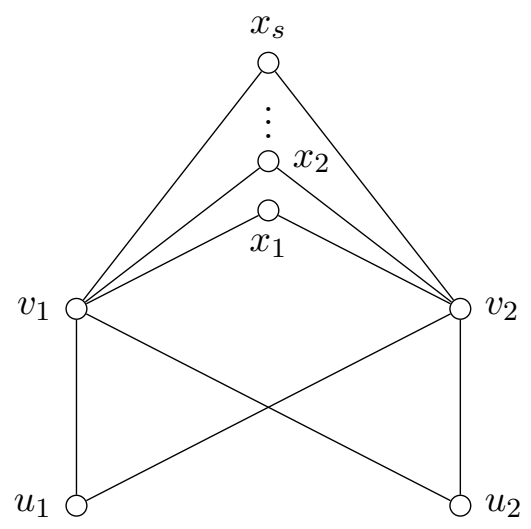

Figure 6.2. The graph $G_{s}$. 


\section{Bibliography}

[1] M. An and L. Xiong, Supereulerian graphs, collapsible graphs and matchings, Acta Mathematicae Applicatae Sinia (English Series), to appear.

[2] M. J. Algefari, K. A. Alsatami, H.-J. Lai and J. Liu, Supereulerian digraphs with given local structures, J. Information Processing Letters, doi: 10.1016/j.ipl.2015.12.008.

[3] J. Bang-Jensen and Thomass'e, unpublished notes.

[4] J. Bang-Jensen and G. Gutin, Digraphs: Theory, Algorithms and Applications, 2nd Edition. Springer-Verlag, London, 2009.

[5] J. Bang-Jensen and A. Maddaloni, Sufficient conditions for a digraph to be supereulerian, J. Graph Theory, 79(1) (2015) 8-20.

[6] C. Berge, Two theorems in graph theory. Proc. Nat. Acad. Sci. USA, 43, (1957) 842-844.

[7] F. T. Boesch, C. Suffel, and R. Tindell, The spanning subgraphs of eulerian graphs, J. Graph Theory, 1, (1977) 79-84.

[8] J. A. Bondy and U. S. R. Murty, Graph Theory. Springer, New York, 2008.

[9] P. A. Catlin, Supereulerian graphs: a survey, J. of Graph Theory, 16, (1992) 177-196.

[10] P. A. Catlin, Reduction method to find spanning Eulerian subgraphs, J. of Graph Theory, 12 , (1988) 29-44.

[11] Z. H. Chen, H.-J. Lai, Reduction techniques for super-Eulerian graphs and related topics-a survey, Combinatorics and graph theory' 95, Vol. 1 (Hefei), World Sci. Publishing, River Edge, NJ 1995 pp. 53-69.

[12] V. Chvátal and P. Erdös, A note on Hamiltonian circuits. Discrete Math., 2, (1972) 111-113.

[13] G. Gutin. Cycles and paths in directed graphs. PhD thesis, School of Mathematics, Tel Aviv University, 1993. 
[14] G. Gutin. Connected (g; f)-factors and supereulerian digraphs. Ars Combin., 54, (2000) 311-317.

[15] L. Han, H.-J. Lai, L. Xiong and H. Yan, The Chvátal-Erdös condition for supereulerian graphs and the Hamiltonian index, Discrete Math, 310, (2010) 2082-2090.

[16] Y. Hong, H.-J. Lai and Q. Liu, Supereulerian Digraphs, Discrete Mathematics, 330, (2014) $87-95$.

[17] H.-J. Lai, Y. Shao and H. Yan, An Update on Supereulerian Graphs, WSEAS Transactions on Mathematics 12, (2013) 926-940.

[18] H.-J. Lai and H. Y. Yan, Supereulerian graphs and matchings, Applied Math. Letters, 24 (2011) 1867-1869.

[19] H. Meyniel, Une condition suffisante d'existence d'un circuit hamiltonien dans un graphe orienté. J. Combin. Theory Ser. B, 14, (1973) 137-147.

[20] W. R. Pulleyblank, A note on graphs spanned by Eulerian graphs, J. Graph Theory, 3, (1979) 309-310.

[21] C. Thomassen. Long cycles in digraphs. Proc. London Math. Soc. (3), 42, (1981) 231-251.

[22] R. Tian and L. Xiong, The Chvátal-Erdös condition for a graph to have a spanning trail, Graphs and Combinatorics, to appear.

[23] J. Xu, P. Li, Z. Miao, K. Wang and H.-J. Lai, Supereulerian graphs with small matching number and 2-connected hamiltonian claw-free graphs, International Journal of Computer Mathematics, 91 (2014), 1662-1672.

[24] L.-C. Zhao and J.-H. Meng. A sufficient condition for hamiltonian cycles in digraphs. Ars Combin., 32:335-338, 1991. 